\title{
Towards Full Aircraft Airframe Noise Prediction: Lattice Boltzmann Simulations
}

\author{
Mehdi R. Khorrami* \\ NASA Langley Research Center, Hampton, Virginia, 23681 \\ and \\ Ehab Fares, ${ }^{\dagger}$ Damiano Casalino ${ }^{\ddagger}$ \\ Exa GmbH, Curiestrasse 4, D-70563 Stuttgart, Germany
}

\begin{abstract}
Computational results for an 18\%-scale, semi-span Gulfstream aircraft model are presented. Exa Corporation's lattice Boltzmann PowerFLOW ${ }^{\circledR}$ solver was used to perform time-dependent simulations of the flow field associated with this high-fidelity aircraft model. The simulations were obtained for free-air at a Mach number of 0.2 with the flap deflected at $39^{\circ}$ (landing configuration). We focused on accurately predicting the prominent noise sources at the flap tips and main landing gear for the two baseline configurations, namely, landing flap setting without and with gear deployed. Capitalizing on the inherently transient nature of the lattice Boltzmann formulation, the complex time-dependent flow features associated with the flap were resolved very accurately and efficiently. To properly simulate the noise sources over a broad frequency range, the tailored grid was very dense near the flap inboard and outboard tips. Extensive comparison of the computed time-averaged and unsteady surface pressures with wind tunnel measurements showed excellent agreement for the global aerodynamic characteristics and the local flow field at the flap inboard and outboard tips and the main landing gear. In particular, the computed fluctuating surface pressure field for the flap agreed well with the measurements in both amplitude and frequency content, indicating that the prominent airframe noise sources at the tips were captured successfully. Gear-flap interaction effects were remarkably well predicted and were shown to affect only the inboard flap tip, altering the steady and unsteady pressure fields in that region. The simulated farfield noise spectra for both baseline configurations, obtained using a Ffowcs-Williams and Hawkings acoustic analogy approach, were shown to be in close agreement with measured values.
\end{abstract}

\section{Nomenclature}

$\begin{array}{ll}\mathrm{AOA} & =\text { angle-of-attack } \\ \mathrm{C}_{\mathrm{L}} & =\text { lift coefficient } \\ \mathrm{Cp}_{\text {rms }}^{\prime} & =\text { unsteady RMS pressure coefficient } \\ \mathrm{Cp} & =\text { pressure coefficient } \\ \mathrm{C} & =\text { local chord } \\ \mathrm{DNS} & =\text { Direct Numerical Simulation } \\ \mathrm{DES} & =\text { Detached Eddy Simulation } \\ \mathrm{MDDES} & =\text { Modified Delayed Detached Eddy Simulation } \\ \mathrm{GAC} & =\text { Gulfstream Aerospace Corporation } \\ \mathrm{Hz} & =\text { Hertz, cycles per second } \\ \mathrm{LES} & =\text { Large Eddy Simulation } \\ \mathrm{M} & =\text { Mach number } \\ \mathrm{PSD} & =\text { power spectral density in }\left[\mathrm{psi}^{2} / \mathrm{Hz}\right] \text { or }[\mathrm{dB} / \mathrm{Hz}] \\ \text { psi } & =\text { pounds per square inch } \\ \mathrm{Re} & =\text { Reynolds number } \\ \mathrm{RMS} & =\text { root mean square }\end{array}$

\footnotetext{
* Aerospace Engineer, Computational AeroSciences Branch, Associate Fellow AIAA

${ }^{\dagger}$ Technical Director, Aerospace Applications, Senior Member AIAA

${ }^{\ddagger}$ Technical Director, Aerospace Aeroacoustics, Member AIAA
} 


$\begin{array}{ll}\text { RNG } & =\text { Re-Normalization Group } \\ \mathrm{S} & =\text { second } \\ \text { URANS } & =\text { unsteady Reynolds averaged Navier-Stokes } \\ \text { VR } & =\text { variable resolution } \\ \mathrm{X}, \mathrm{Y}, \mathrm{Z} & =\text { right handed coordinate system }\end{array}$

\section{Introduction}

Aircraft noise has been recognized as an environmental pollution problem since the advent of commercial air transportation. Today, it is a hazard that affects metropolitan areas adjacent to major airports. With the projected growth in air travel and the relentless pace of urban development, community exposure to substantially higher noise levels seems inevitable. NASA's Environmentally Responsible Aviation (ERA) project is pursuing the development, evaluation, and maturation of novel noise reduction technologies that aim to confine aircraft noise footprints on the ground within airport boundaries. This is to be accomplished without detrimental effects to the aerodynamic efficiency of aircraft. To most people, aircraft noise is synonymous with jet engine noise. During approach to landing, however, noise generated by the airframe is comparable to, and in some instances even louder than, propulsion noise. Acoustic measurements from flight tests ${ }^{1-6}$ and model-scale experiments ${ }^{7-8}$ have identified the landing gear and lift enhancing devices, such as wing slats and flaps, as the prominent sources of airframe noise. Efficient design and development of viable noise reduction technologies demand the availability of high-fidelity simulation tools with accurate predictive capabilities. Ultimately, the goal of creating such tools is to promote a paradigm shift in the design procedure from the current time consuming and costly trial and error approach to a physics-based virtual design environment whereby the aeroacoustic evaluation of a noise reduction concept and its subsequent optimization can take place in an integrated fashion.

As part of the NASA-Gulfstream partnership on airframe noise research, a series of flight tests and model scale experiments are being conducted with a Gulfstream aircraft as the baseline configuration. An 18\% scale, semi-span replica of the chosen aircraft was designed and fabricated specifically to conduct airframe noise studies and evaluate noise reduction concepts for mitigating landing gear, flap, and gear-flap interaction noise. Aeroacoustic testing of the $18 \%$ scale semi-span model was performed as a series of carefully planned entries in the NASA Langley Research Center (LaRC) 14- by 22-Foot Subsonic Wind Tunnel (14x22). The initial entry, completed in November 2010, focused on acquiring global forces (lift and drag) and measurements of steady and unsteady surface pressures. Detailed accounts of that entry and the processed aerodynamic data are given in Khorrami et al. ${ }^{9}$ and Khorrami and Neuhart. ${ }^{10}$ The second $14 \times 22$ tunnel entry was executed in two segments. The first segment was dedicated to simultaneous acoustic and surface pressure measurements, ${ }^{11,12}$ while the second segment was devoted to off-surface flow measurements for the nominal aircraft landing configuration. ${ }^{13}$

Concurrent with the experimental test campaigns, there is a comprehensive computational effort aimed at extending the state-of-the-art in airframe noise prediction capabilities from component-level to full aircraft systemlevel configurations. A unique aspect of the NASA-Gulfstream collaborative effort is the complementary, and sometimes leading, role that the computational simulations play in the process toward better design, evaluation, testing, and down-selection of noise reduction technologies. The computational effort is built upon the knowledge and experience gained from previous aeroacoustic simulations of an isolated nose landing gear for a Gulfstream aircraft and other relevant configurations. ${ }^{14-19}$ Following those earlier simulations, the current full-aircraft airframe noise prediction effort is being pursued via execution of two distinct computational methodologies. In this paper, results obtained with Exa Corporation's PowerFLOW ${ }^{\circledR}$ solver are presented. The code, based on a subsonic compressible lattice Boltzmann Method (LBM) flow solver, was used to perform unsteady simulations of the 18\% scale, semi-span Gulfstream aircraft model in landing configuration (with the main landing gear off and on). In a companion paper by Khorrami and Mineck, ${ }^{20}$ NASA's FUN3D unstructured compressible Navier-Stokes solver was used to conduct Detached Eddy Simulations (DES) of the same model.

\section{Simulated Model Geometry}

The simulated model geometry corresponds to an $18 \%$ scale, semi-span high-fidelity reproduction of a Gulfstream aircraft (see Fig. 1). The model geometry consists of a fuselage, wing, flap, flow-through nacelle, pylon, and main landing gear. A full description of this model, including the surface distribution of steady pressure ports and unsteady transducers on various components, is provided in Refs. 9 and 10 . The baseline case of $39^{\circ}$ flap deflection without the main landing gear was used to perform an extensive computational study focused on mesh resolution and solution convergence. Figures 2 and 3, reproduced from Ref. 9, show the top view of the spanwise 
location and numbering of the static pressure rows on the model wing, winglet, and flap. Note in Fig. 2a that the wing pressure rows extend to include the area that overlaps the stowed flap top surface, shown in purple. This darker region represents the wing spoiler, which was treated as part of the wing for instrumentation purposes.

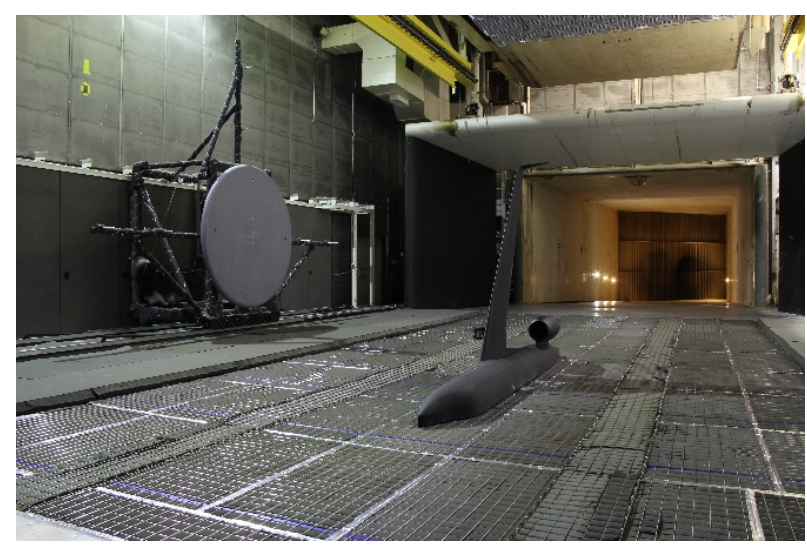

a) Installed in NASA LaRC 14x22 wind tunnel

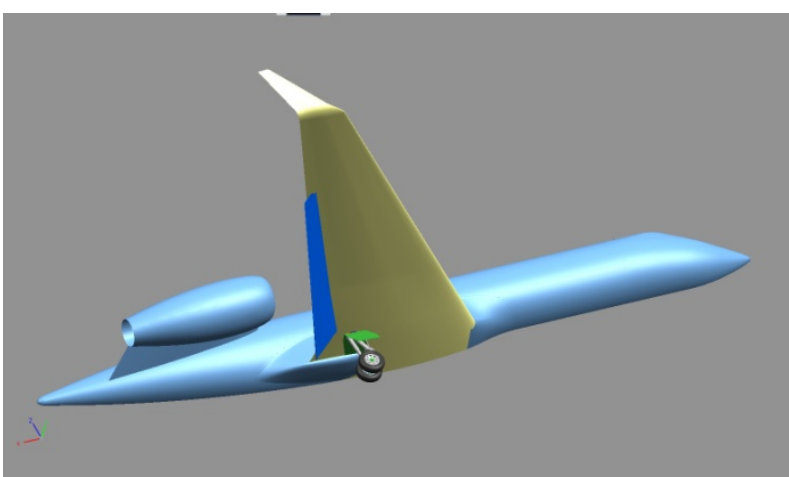

b) CAD model

Figure 1. 18\% scale semi-span model of a Gulfstream aircraft.

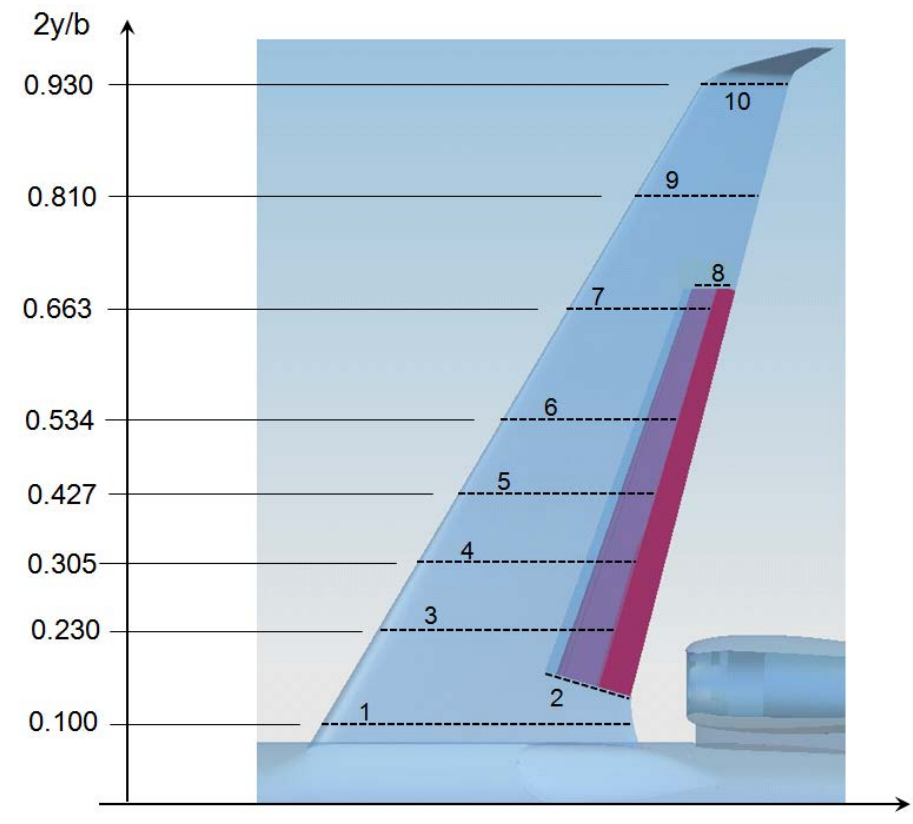

a) Wing

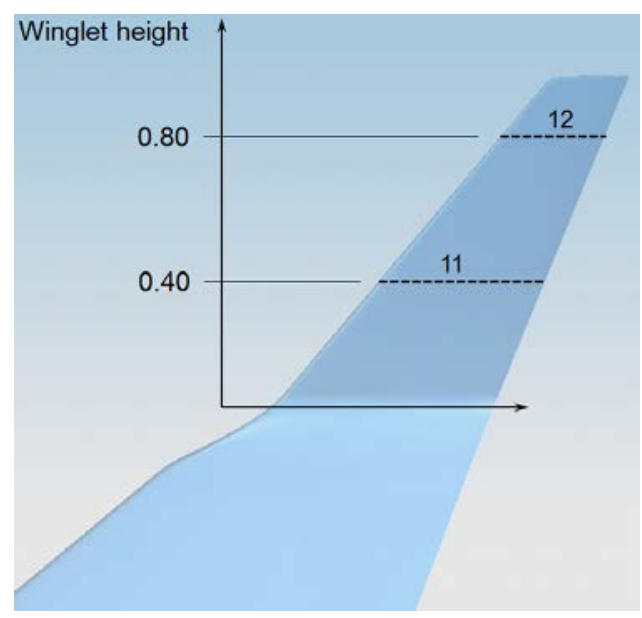

b) Winglet

Figure 2. Spanwise location and identification number for rows of static pressure orifices (from Ref. 9). 


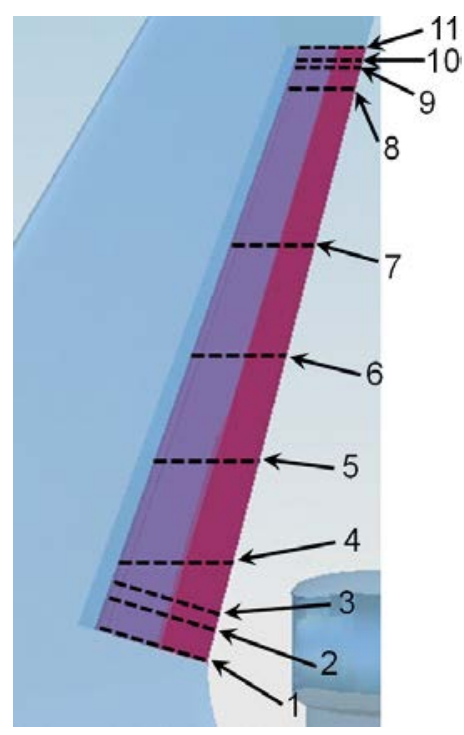

Figure 3. Flap orifice row distribution and numbering (from Ref. 9).

Figures 4 through 8, taken from Ref. 10, display the locations and numbering of the unsteady pressure sensors on the model flap and main landing gear (including the gear cavity door) used extensively to validate the present computations. Altogether, the flap has 40 dynamic sensors. The sensors (herein after referred to as probes) are distributed in three distinct areas: along the mid-span section (not shown), and two narrow chordwise strips adjacent to the inboard and outboard edges where significant flow unsteadiness is expected.

The probe locations for the inboard and outboard tips are shown in Figs. 4 and 5, respectively. Note that the flap side-edge has a cavity that nearly spans the entire chord length. In the actual aircraft, this cavity houses a bulb seal that prevents metal to metal contact during flap retraction. To document whether the cavity acoustic modes get excited at high flap deflections, two probes (P26 and P27) are installed inside the cavity. The simulated geometry also includes this cavity and the bulb seal within it.

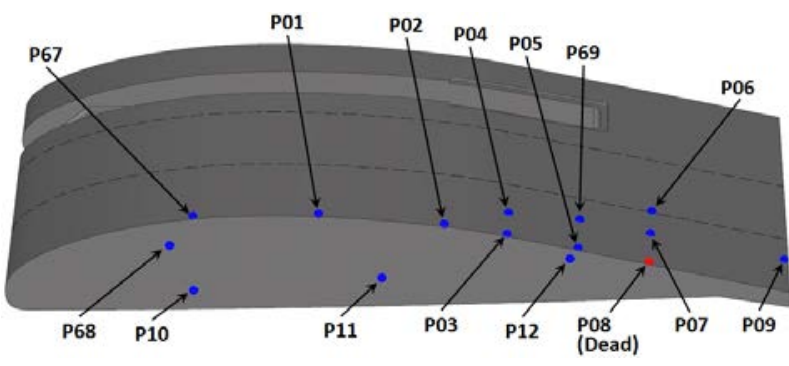

a) Top and side view

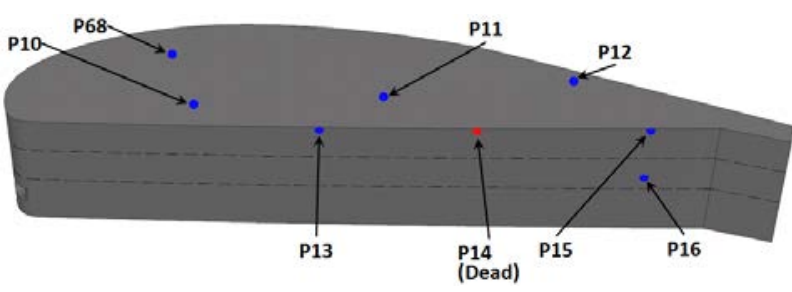

b) Bottom and side view

Figure 4. Locations and numbering of probes installed at the flap inboard edge (from Ref. 10).

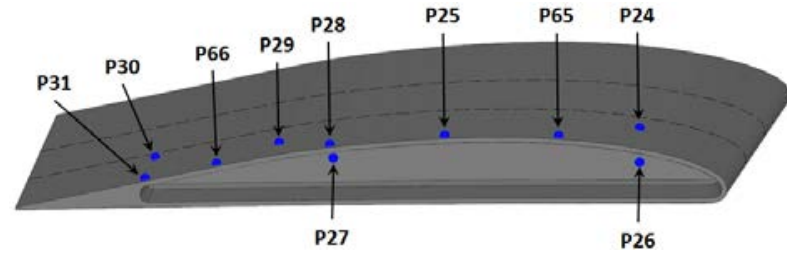

a) Top and side view

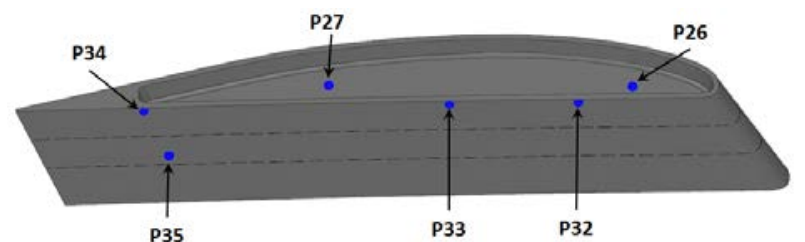

b) Bottom and side view

Figure 5. Locations and numbering of probes installed at the flap outboard edge (from Ref. 10). 


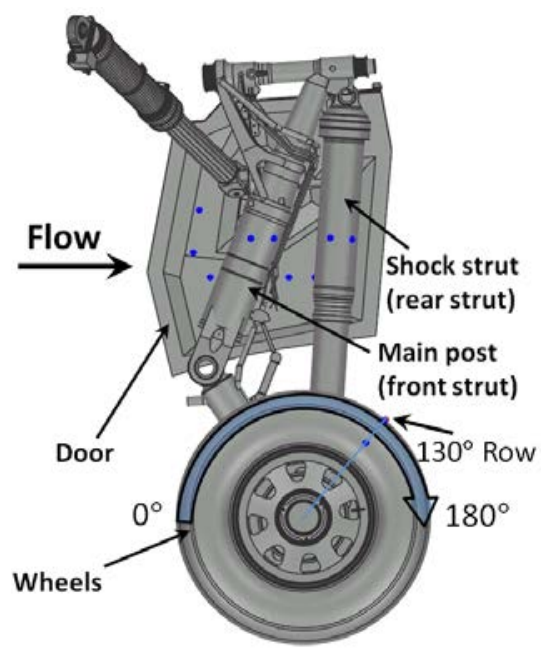

a) Side view

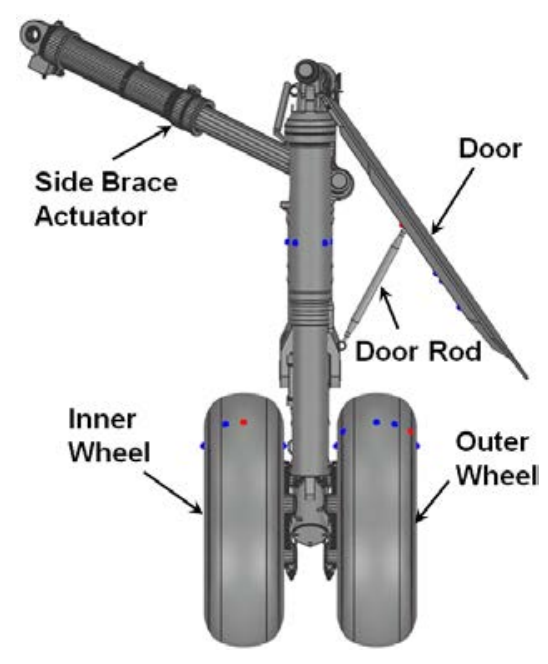

b) Rear view

Figure 6. Side and rear views of the main landing gear highlighting major components (from Ref. 10).

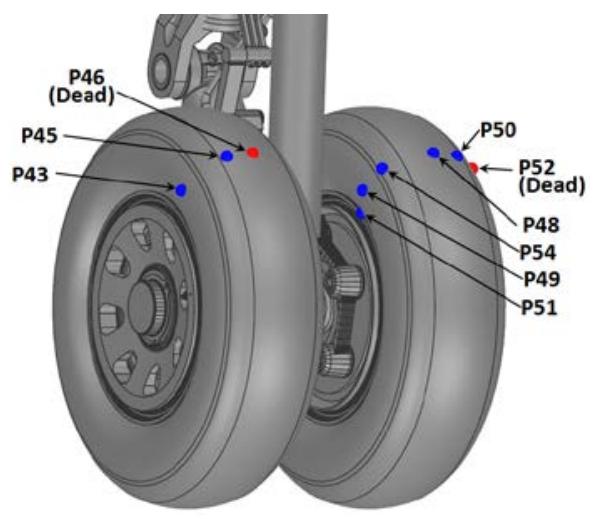

a) Interior (fuselage) view

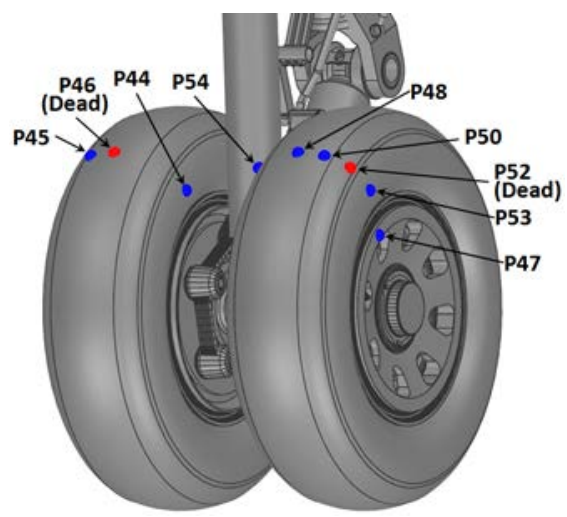

b) Exterior (wing-tip) view

Figure 7. Interior and exterior views of the wheels showing locations of installed probes (from Ref. 10).

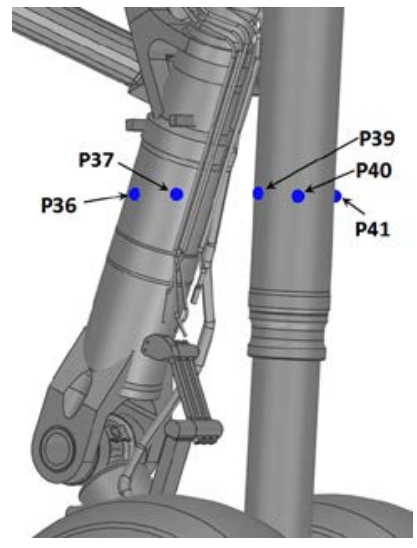

a) Interior (fuselage) view

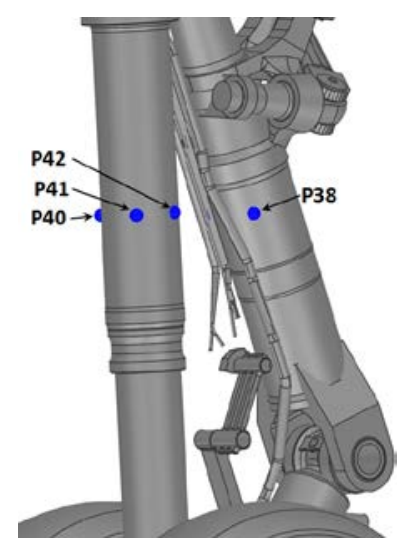

b) Exterior (wing-tip) view

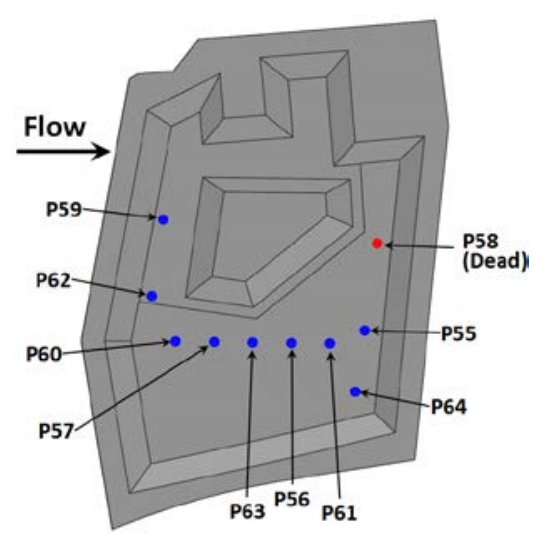

c) Door interior

Figure 8. Interior and exterior views of main gear post, shock strut, and door (from Ref. 10). 


\section{Computational Approach}

The numerical simulations documented in this paper were performed using Exa Corporation's commercial solver PowerFLOW ${ }^{\circledR}$ versions 4.4b and 5.0, which are based on the three-dimensional 19 state (D3Q19) lattice Boltzmann model. The software has been successfully validated for flows over highly complex geometries such as those reported in Refs. 16 through 19.

\section{A. Flow Solver}

The lattice Boltzmann equations represent an alternative mesoscopic formulation to the classical macroscopic Navier-Stokes equations describing a compressible unsteady flow of a continuum. The details of the mathematical foundations are documented in references 18, 19 and 21. The simple, local formulation of the underlying D3Q19 equations allows a highly efficient implementation for distributed computations on thousands of processors. The low dissipation and dispersion properties of the numerical scheme typically produce aerodynamic and aeroacoustic results that are comparable to those obtained with classical CFD solvers that use higher-order large eddy simulation (LES), as demonstrated in the comparative study of flow over tandem cylinders presented in Ref. 22.

\section{B. Turbulence Modeling}

The lattice Boltzmann flow simulation is equivalent to a Direct Numerical Simulation (DNS) of the flow. For high Reynolds number flows, such as those addressed in this work, turbulence modeling is incorporated into the lattice Boltzmann model by replacing the relaxation time, which is related to the thermodynamic chaotic motion of fluid particles, by a turbulent relaxation time that models the effect of turbulent chaotic motion on the statistics of fluid particle collisions. The turbulent kinetic energy and the turbulent dissipation are obtained by solving a variant of the RNG k- $\varepsilon$ model. ${ }^{23}$ This model incorporates a swirl term that detects the occurrence of large-scale threedimensional turbulent structures and reduces the influence of the turbulence model on the simulated flow accordingly. In this respect, the turbulent lattice Boltzmann flow model is analogous to a hybrid URANS-LES formulation, with the difference that, compared for instance to standard DES formulations, the blending between LES and URANS is based on the resolved turbulence structures and has therefore an adaptive character both in space and time. ${ }^{24}$ To reduce the resolution requirements near the wall for high Reynolds number flows, an extended wall function is used to model the boundary layer on solid surfaces. The wall function model is an extension of the standard formulation and includes the effects of favorable and adverse pressure gradients on the outer boundarylayer variables.

\section{Computational Grids}

The lattice Boltzmann formulation is solved on Cartesian meshes that are generated automatically within PowerFLOW ${ }^{\circledR}$ for any geometrically complex shape. This greatly simplifies the labor-intensive volume meshing step usually associated with other approaches. Thirteen variable refinement (VR) regions are defined to allow local mesh refinement by successive factors of 2 . The axis system was aligned with the vehicle body axis system (X along the line from the nose to the tail, $\mathrm{Y}$ from the centerline to the right wing tip, and $\mathrm{Z}$ pointing away from the ground, a right handed axis system). Locations and distances are presented in non-dimensional grid units (one grid unit equals one inch). The nose of the fuselage was located at $(-0.72,-3.50,13.95)$, the end of the fuselage was located at $(184.68,-3.50,20.37)$, and the tip of the winglet was located at $(146.11,100.82,23.86)$. A series of grids was generated to demonstrate grid convergence and to efficiently cluster the mesh around the wing surface for an accurate resolution of the flap side edge flow. Offset VR regions were used to achieve $\mathrm{y}^{+}$values below 150 around the wing and approximately 20 near the flap tips. Additional off-body VR regions were also included to resolve the wing tip vortex and both inboard and outboard flap tip vortex formation regions as depicted in Figs. 9 and 10. The Cartesian volume element (voxel) size on the inboard flap side edge surface was $0.1875 \mathrm{~mm}$, whereas the outboard flap tip VR offset region had a voxel size of $0.0938 \mathrm{~mm}$ constituting the smallest voxel size of the volume mesh. The inboard and outboard flap tip vortex rollup regions were resolved using $0.75 \mathrm{~mm}$ and $0.1875 \mathrm{~mm}$ resolutions, respectively.

The aircraft surface was subdivided into different patches (PIDs) as depicted for the flap surface in Fig. 11 in order to distinguish between acoustic contributions from different regions during the subsequent farfield acoustic propagation computations. The generated grid had a total of $2.6 \times 10^{9}$ voxels and $40 \times 10^{6}$ surface elements (surfels). The simulation was executed for $4.5 \times 10^{6}$ time steps to cover a physical time of $0.7 \mathrm{~s}$. For the grid resolution study additional medium and coarse meshes, comprised of globally larger voxel sizes of factor 1.5 and 2.25, respectively, were used. 


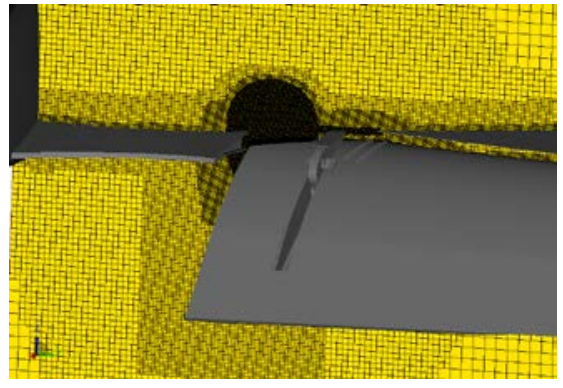

a) Spanwise cut near leading edge

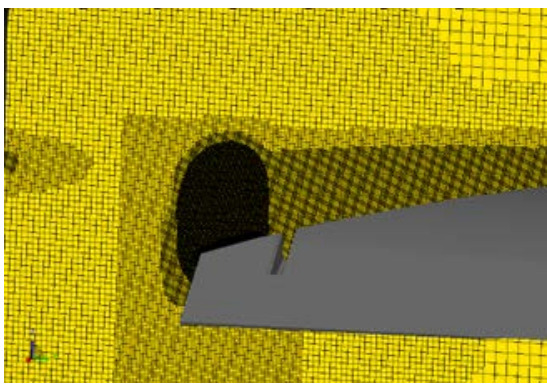

b) Spanwise cut near mid-chord

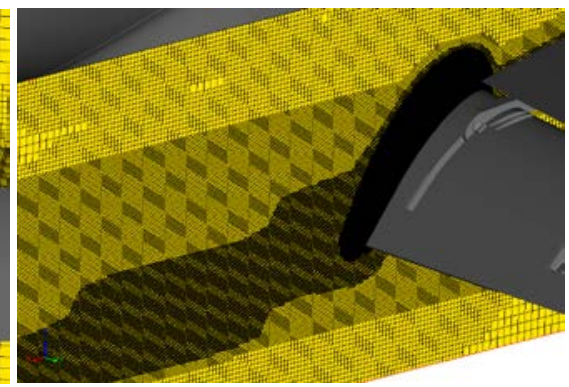

c) Streamwise cut near tip

Figure 9. Close-up view of model geometry near flap inboard tip and corresponding volume mesh (every second line is shown).

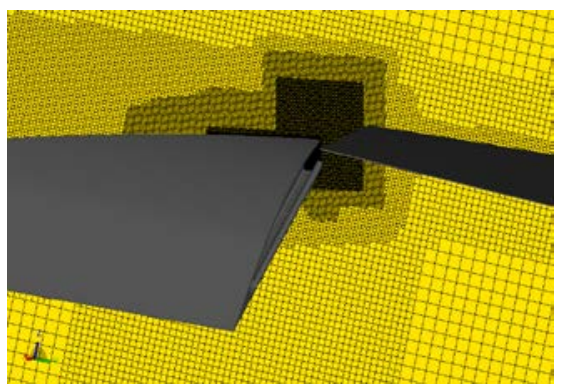

a) Spanwise cut near leading edge

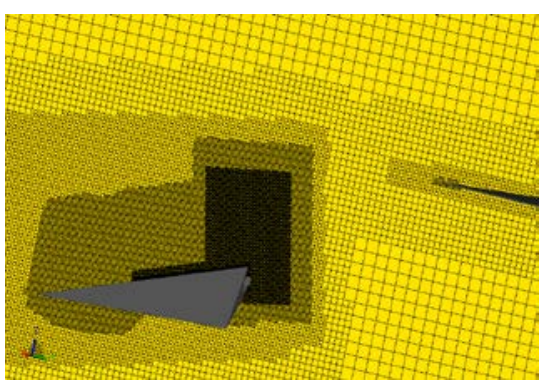

b) Spanwise cut near mid-chord

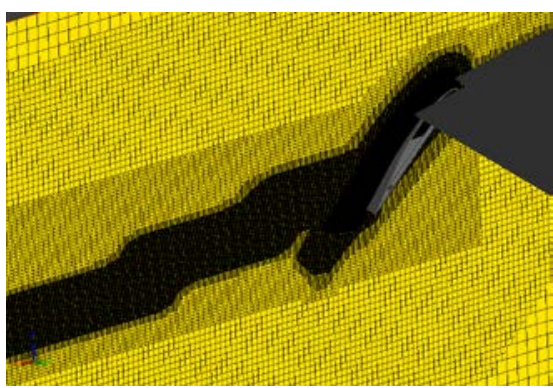

c) Streamwise cut near tip

Figure 10. Close-up view of model geometry near flap outboard tip and corresponding volume mesh (every second line is shown).

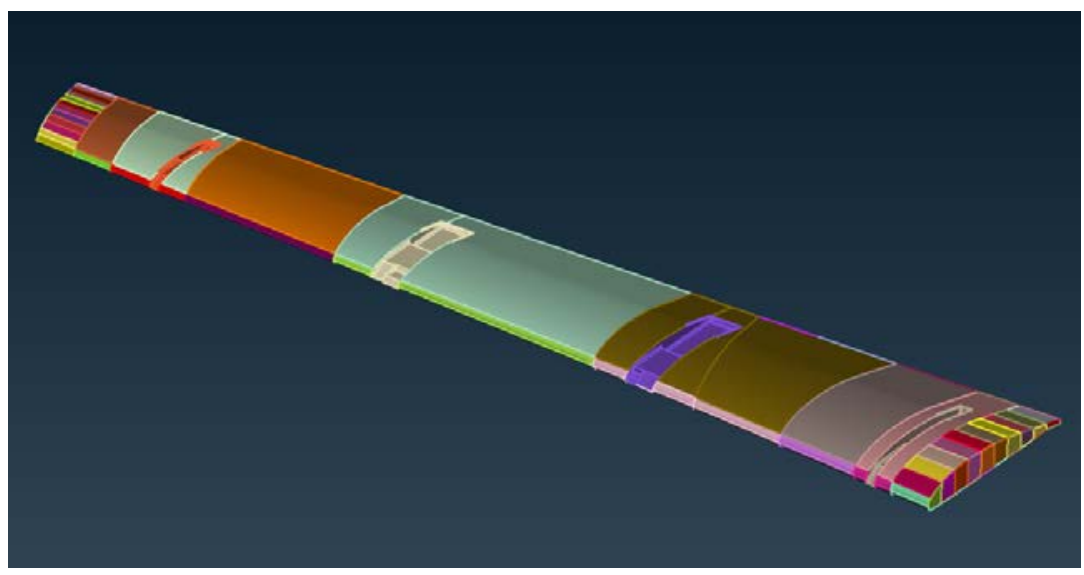

Figure 11. Flap surface composed of various surface patches for subsequent acoustic farfield propagation contributions.

Once a satisfactory meshing strategy and the requisite mesh spacing were established, attention was focused on the baseline configuration with the main landing gear deployed. Due to the automatic meshing capabilities of PowerFLOW ${ }^{\circledR}$, inclusion of the main landing gear in the simulations did not impact the existing mesh around other regions of the aircraft, thus allowing us to make consistent comparisons between baseline configurations with and without the gear. Only a local change of the mesh was required by adding variable resolution regions around the gear and its bay that are included in the computer-aided design (CAD) model of the aircraft. Figure 12 shows the 
main landing gear deployed under the wing and the wheel bay. The relative position between the gear and the wing flap is such that a strong interaction is expected between the gear wake and the inboard flap edge. The manner in which this interaction is manifested may impact the effectiveness of any low-noise concept applied to the inboard tip. Figure 13 shows the mesh layout past the gear components. The finest volume resolution was obtained by an offset of about $1.85 \mathrm{~mm}$ of most of the parts and setting a voxel size of about $0.18 \mathrm{~mm}$, thus corresponding to a mesh layer of about 10 voxels. The mesh was progressively coarsened away from the gear; and, to properly capture the gear wake and resolve its interaction with the flap, a mesh resolution of $3 \mathrm{~mm}$ was applied to a box that extends three wheel diameters downstream of the gear. This approach generally follows the same best practice developed previously for landing gear simulations as documented in Ref. 19. The generated fine grid had a total of $2.9 \times 10^{9}$ voxels and $57 \times 10^{6}$ surfels.
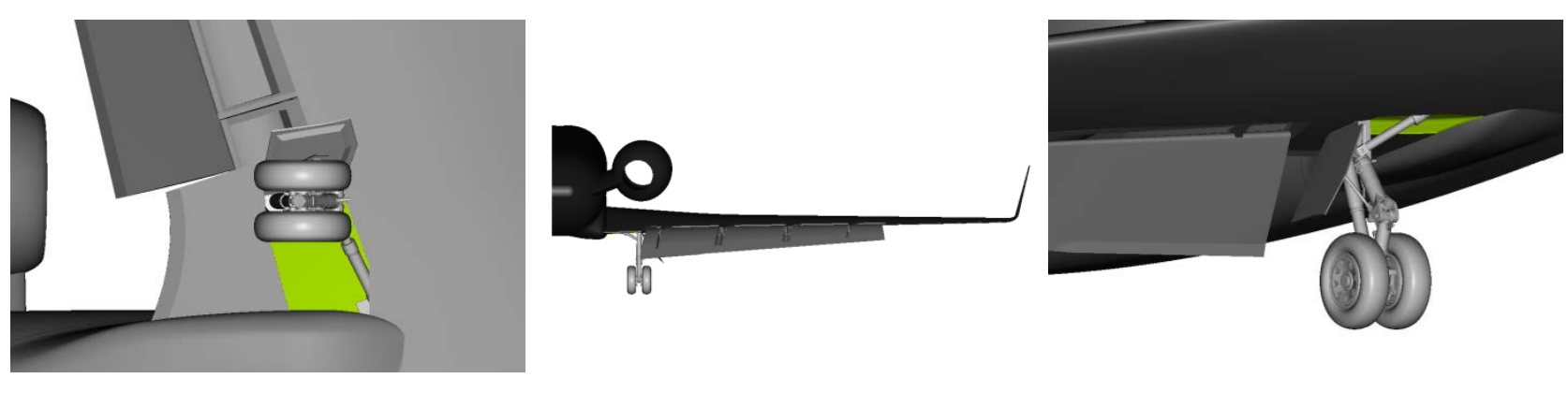

Figure 12. Views of the main landing gear and its bay highlighted in green.
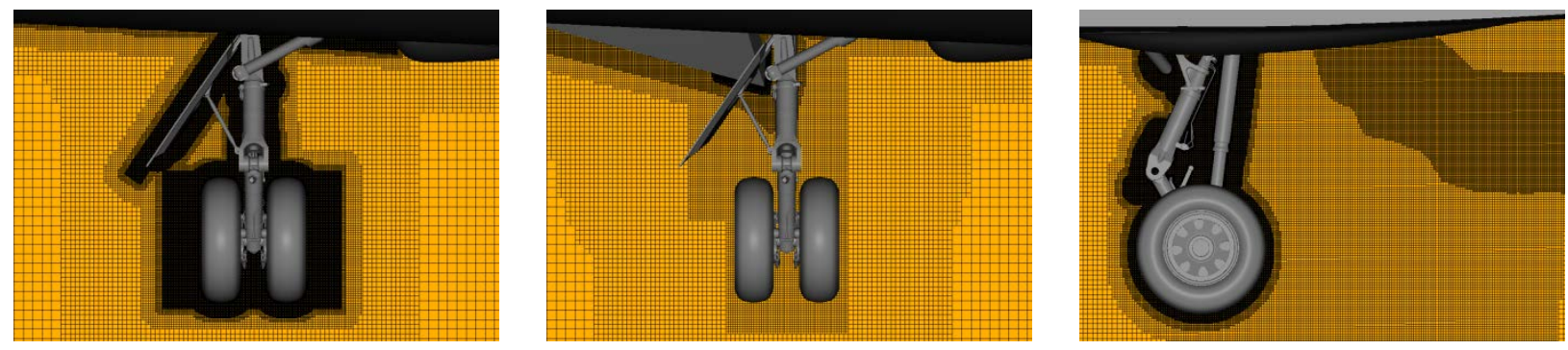

Figure 13. Close-up views of the computational mesh past the gear (every second line is shown)

\section{Boundary Conditions}

All aircraft surfaces and the viscous floor region were modeled with no slip boundary conditions via a generalized bounce back volumetric formulation ${ }^{25,26}$ near the wall for arbitrarily oriented surfels within the voxels. The remaining portion of the floor was modeled as a symmetry plane. Velocity and turbulent kinetic energy were imposed at the inflow boundaries, whereas the static pressure was kept constant at the outflow. Other values were extrapolated from the simulation domain. The boundary conditions were used in an under-relaxed manner to avoid large local gradients especially during the startup process. In addition, sponge layer zones were included in the farfield to damp reflections of acoustic waves.

\section{E. Computational Procedure}

PowerFLOW ${ }^{\circledR}$ simulations were initialized with freestream conditions for a period corresponding to the time that it would take the flow to convect a distance of 40 mean aerodynamic chords. The time steps $(\Delta t)$ used for the current set of simulations were $1.539 \times 10^{-7} \mathrm{~s}, 2.309 \times 10^{-7} \mathrm{~s}$, and $3.079 \times 10^{-7} \mathrm{~s}$ corresponding to the fine, medium, and coarse grids, respectively. The statistical convergence of the flow solution during this initial transient time was monitored using unsteady pressure signals acquired on the surface of the wing and the time-history of the global aerodynamic forces acting on the aircraft.

After reaching statistical convergence, the simulation was executed over $0.3 \mathrm{~s}$ of physical time; then, the computed flow variables were stored in a number of different volume and surface measurement files for subsequent 
flow analysis and post-processing. The flow variables were stored with various sampling rates, ranging from a sampling frequency of $200 \mathrm{kHz}$ for the surface to $100 \mathrm{kHz}$ in some areas of the volume. Post-processing spectral analyses were conducted using a bandwidth of $20 \mathrm{~Hz}$ and an overlapping coefficient of 0.5. Results were compared with experiment up to $40 \mathrm{kHz}$.

An acoustic analogy approach based on the Ffowcs-Williams and Hawkings (FWH) formulation ${ }^{27}$ was used to extrapolate the computed near-field fluctuations to the farfield. The employed FWH formulation was based on the retarded-time formulation $1 \mathrm{~A}$ by Farassat, ${ }^{28}$ extended to account for uniform mean flow convection effects to simulate the noise generated and measured in an ideal infinite wind tunnel. ${ }^{29}$ Integrations on different solid surfaces of the aircraft and on permeable surfaces surrounding different components of the aircraft were performed to evaluate the relative contribution to the overall noise by different wing and high-lift device (HLD) components, and to quantify the levels of noise generated by turbulent fluctuations within boundary and shear layers directly radiated into the farfield as quadrupole noise.

\section{Results and Discussion}

During the 201014 x 22 wind tunnel entry, aerodynamic measurements of the $18 \%$ scale, semi-span model were obtained with the tunnel in both closed (hard) wall and open-wall (open-jet) configurations. As demonstrated in Khorrami et al., ${ }^{9}$ the wall-corrected model aerodynamic forces (i.e., lift coefficient) representing free-air conditions were in good agreement with the open-jet data, with the latter configuration producing slightly less lift at all anglesof-attack (AOA) (see Fig.14). For the baseline landing configurations (with or without the main gear), the fluctuating surface pressures on the model flap and main landing gear were virtually identical for both tunnel configurations. In addition, the acoustic and off-surface (particle image velocimetry) flow measurements required that the tunnel be in an open-wall (open-jet) configuration. Therefore, a decision was made to conduct the majority of the computations with the model in a free-air setting. All the initial simulations were performed for AOA of $3^{\circ}$, flap deflection angle of $39^{\circ}$ (landing configuration), and with the main landing gear removed. This configuration provided an excellent intermediate geometry for benchmarking our computational approach before the more resource intensive configuration involving the main landing gear was attempted.

The computations in PowerFLOW $\AA$ are performed in a non-dimensional fashion in lattice units and transformed later to physical units or any other standard non-dimensional scaling. The scales used in the normalizations are the freestream speed of sound, density, kinematic viscosity, and a unit length of smallest voxel size. For the present case, inlet reference flow variables were set to match conditions at the $14 \times 22$ wind tunnel test section entrance. All simulations were

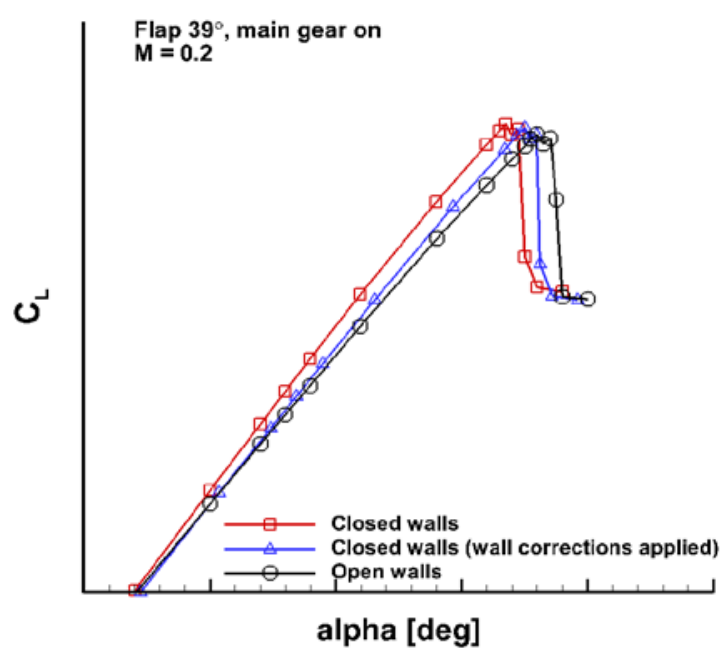

Figure 14. Tunnel configuration effects on lift coefficient.

obtained for a freestream Mach number of 0.2 . The corresponding unit Reynolds number is $1.33 \times 10^{6}$ per foot $(4.40$ x $10^{6}$ per meter), resulting in a Reynolds number of $3.40 \times 10^{6}$ based on the $18 \%$ scale model mean aerodynamic chord of 30.8 in $(0.782 \mathrm{~m})$. Because both upper and lower surfaces of the wing were tripped in the experiment, ${ }^{9}$ the computations were conducted with specified laminar patches on the main wing leading edge upstream of the actual tripping locations. A similar procedure was followed in the vicinity of the fuselage nose.

The initial computations were mostly used to a) observe solution convergence behavior for the model integrated forces such as lift and drag coefficients, b) identify under-resolved flow regions and further refine the grid, and c) achieve a modest level of grid independence for the computed solution (changes in lift and drag coefficients within $1 \%$ for each successive refinement). A full account of these initial runs is beyond the scope of the present paper. We will limit ourselves to describing the major findings that guided our choices of grid setup and mesh resolution.

\section{A. Global View}

Before proceeding to quantitative comparisons between localized measurements and computations, a global picture of the flow field is presented. Most of the flow unsteadiness originates from the flap tip regions. Although 
the simulated flap geometry is very complex, including several brackets and tracks, worm gears, and vortex generators, the major sources of flow unsteadiness reside mainly in the vicinity of the two flap side edges. Figure 15 shows a representative plot of computed time-averaged surface pressure distribution over the semi-span model with the flap deployed. The surface pressures display the footprint of strong suction peaks at the flap edges, especially the inboard tip; these peaks are attributed to the presence of strong streamwise vortices. Also evident in Fig. 15 is an alteration of the leading edge suction peak due to the presence of the flap brackets.

\section{1. $\quad$ Flap Inboard Tip}

A close-up view of the inboard edge showing instantaneous vortex formation and the resulting fluctuating pressure field on the surface of the aircraft is displayed in Fig. 16. The existence of a strong pressure differential between the bottom and top surfaces of the flap results in the formation of a complex dual-vortex system. Near the flap leading edge, the boundary layer on the bottom surface separates at the sharp corner. The separated shear layers at the flap tips are rapidly deformed by Kelvin-Helmholtz instabilities, resulting in the formation of numerous vortex filaments of differing sizes and shapes. Roll-up of the deformed shear layers produces two prominent streamwise vortices situated along the lower and upper edges of the flap tip. Both vortices gain strength and size along the flap chord due to the continuous roll-up of the detached shear layers at the two sharp edges. Downstream of the flap mid-chord, the lower side vortex begins to interact and merge with the vortex on the top surface. Eventually, a single dominant streamwise vortex is formed. The intricate vortex formation process is captured in the close-up view of the unsteady and time averaged flow field at the inboard edge presented in Fig. 17.

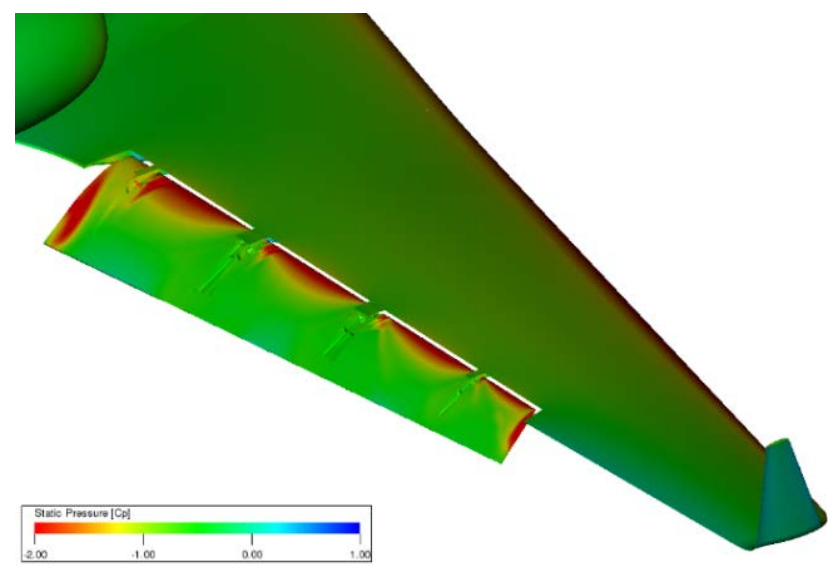

Figure 15. Computed steady surface pressure distribution on $18 \%$ scale, semi-span model with flap deployed.

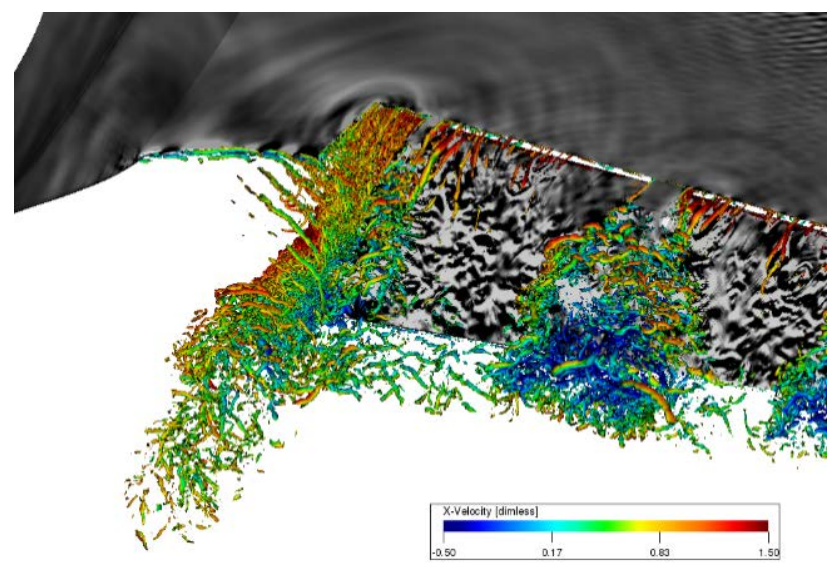

Figure 16. Close-up view of the inboard flap edge showing instantaneous flow field based on isosurface of the $\lambda_{2}$ criterion (at -3000 ) and surface dilatation field. 

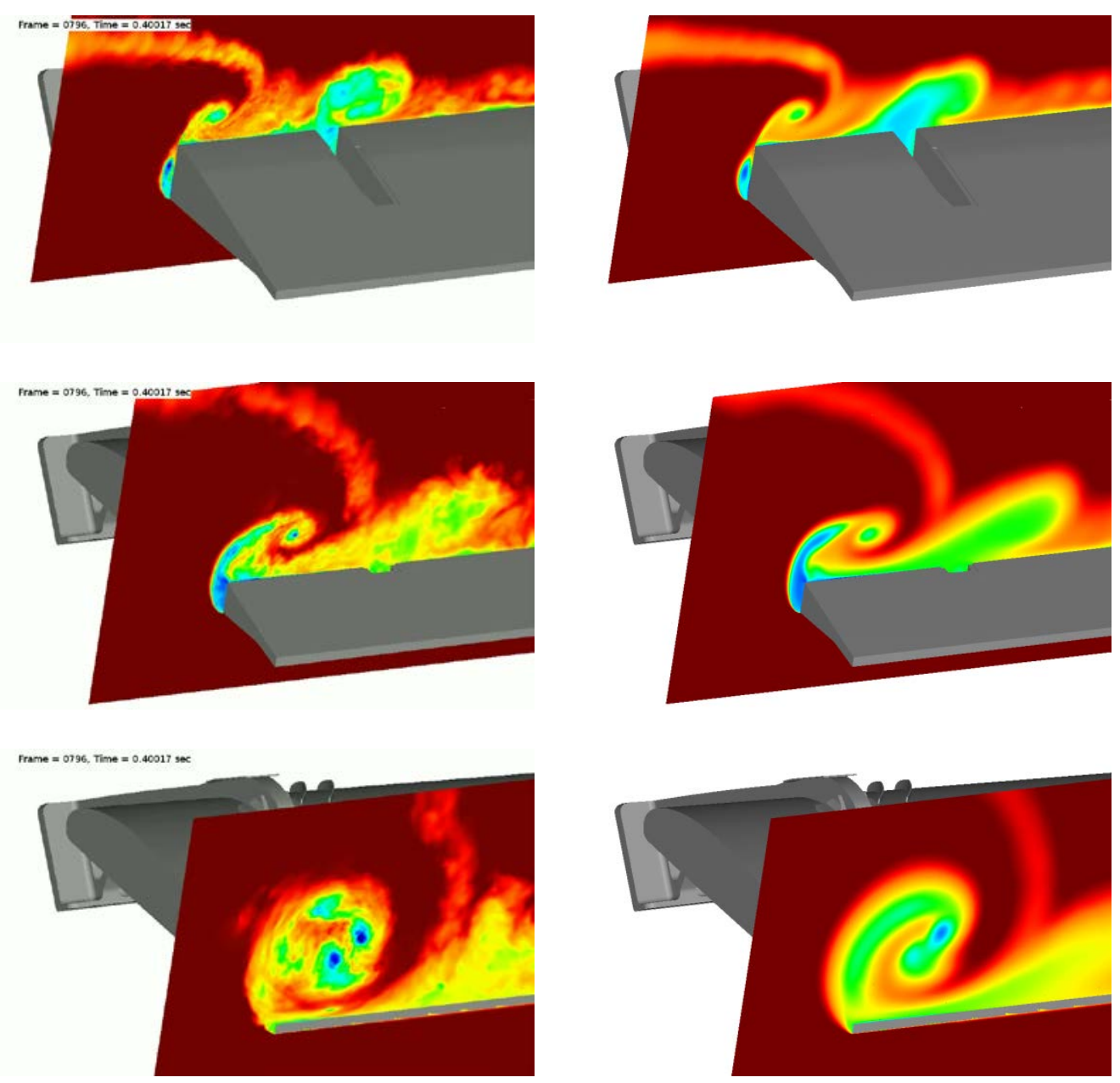

Figure 17. Total pressure contours of unsteady (left) and time-averaged (right) flow at the inboard flap side edge at three streamwise locations along chord.

\section{Flap Outboard Tip}

In contrast to the inboard tip, the outboard tip does not possess a straight-edge side wall that is typical of the simple model-scale geometries studied previously. Since the outboard tip houses a cavity and a bulb seal, its flow field should be quite complex and different than that described in the previous section for the standard straight-edge tip. However, the geometry of the outboard tip should not be viewed as being unique. Many of the civil transports in service today have flap tips with cavities, cut outs, bracket rollers, and other depressions for flap deployment purposes.

A close-up view of the flow field at the outboard tip is shown in Fig. 18. The break-up of the separated shear layer at the edge into three-dimensional vortex filaments and their subsequent roll-up into the tip vortex is evident in this figure. The vortex filament colors represent the strength of the local streamwise velocity; thus, the red filaments near the edge indicate an accelerating streamwise flow that significantly exceeds the freestream speed by nearly $50 \%$. Spanwise slices of the instantaneous and time-averaged pressure fields at the outboard tip are shown in Fig. 19. Similar to the inboard tip, the pressure field depicts the formation of a dual vortex system with the vortex at the top edge much weaker and not lasting long along the chord before it is engulfed by and pulled into the larger and stronger side-edge vortex. However, emergence of the vortical structures is not as well defined as for the inboard edge, in particular near the leading edge. Clearly, the bulb seal and interaction of the side edge vortex with the tip cavity alter the flow field dynamics, shear layer roll-up, and how the two vortices merge. 


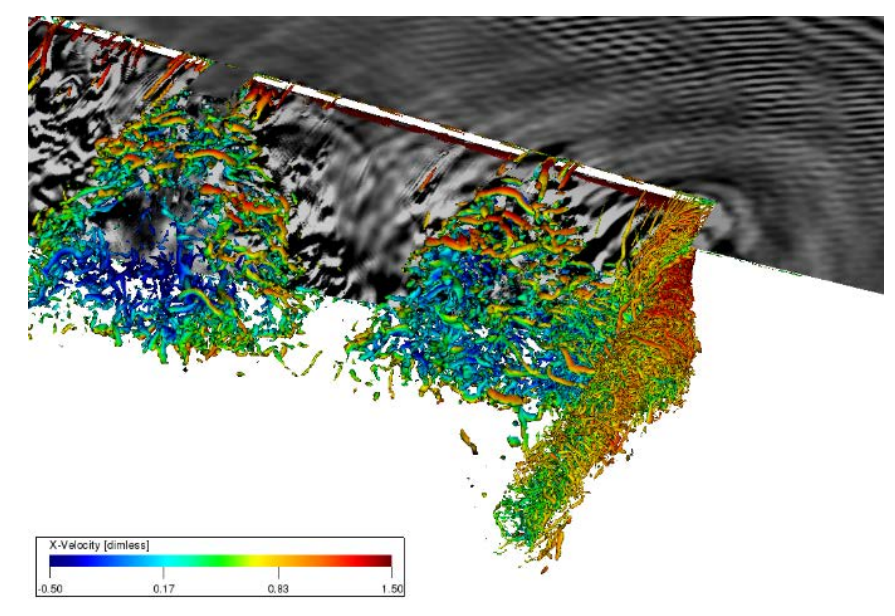

Figure 18. Close-up view of the outboard flap edge showing instantaneous flow field based on isosurface of the $\lambda_{2}$ criterion (at -3000 ) and surface dilatation field.
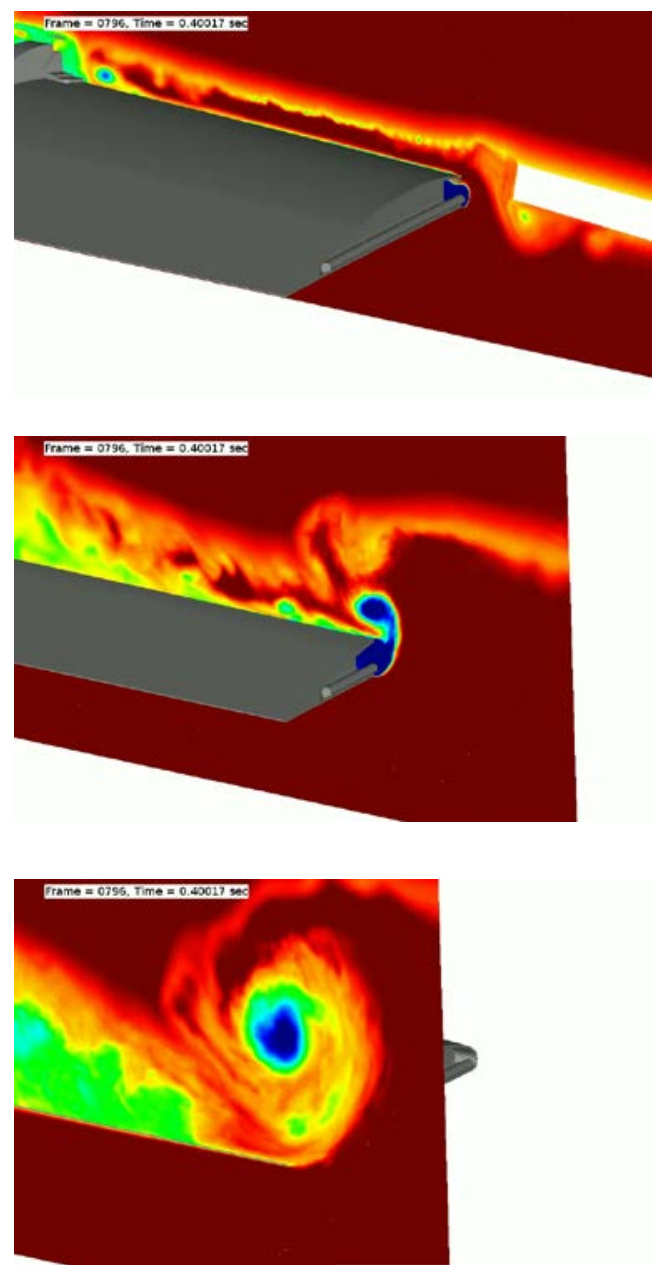
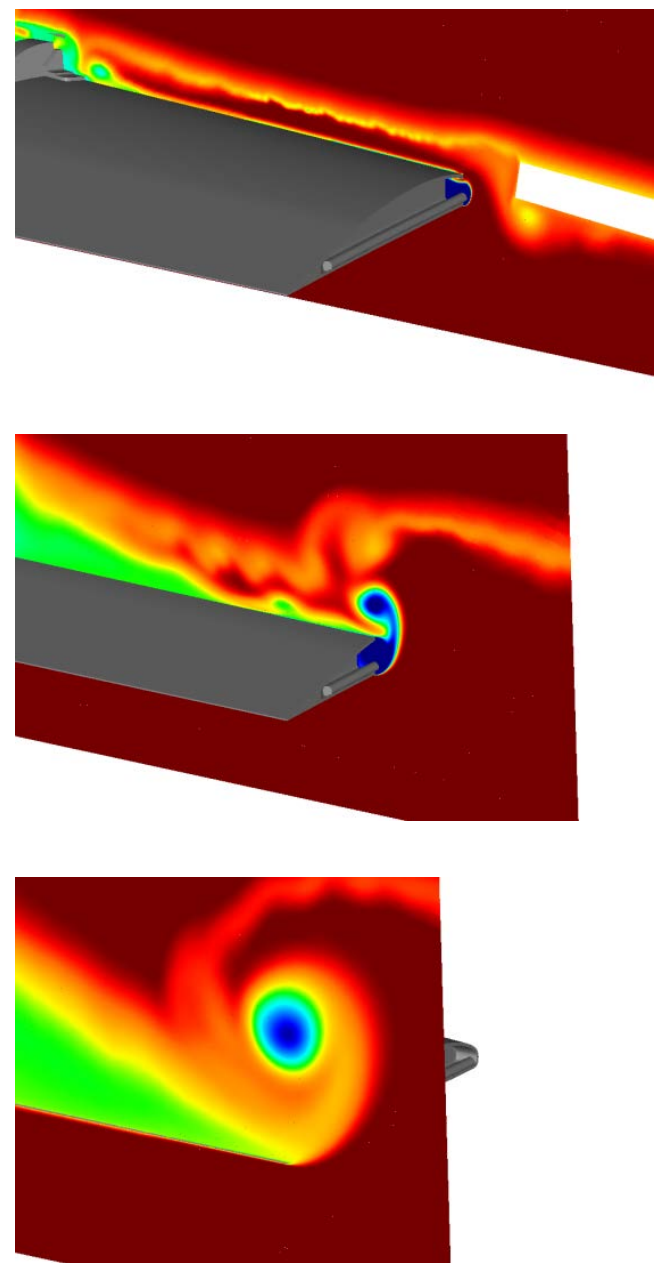

Figure 19. Total pressure contours of unsteady (left) and time averaged (right) flow at the outboard flap side edge at three streamwise locations along chord. 


\section{Main Landing Gear}

The main landing gear impacts the overall aerodynamic performance of the aircraft by increasing the drag and by affecting global circulation. The latter effect is expected to be of secondary importance, as will be discussed in the following sections. Because of the main landing gear location under the wing, its wake is convected past the inboard portion of the flap, where a more significant localized effect is expected. An instantaneous snapshot of the vorticity field in the region containing the gear and inboard flap tip is presented in Fig. 20. The figure shows the presence of turbulent flow structures in the form of vortex filaments of various shapes, sizes, and strengths being shed from different gear subcomponents. These structures are the source of the broadband surface pressure fluctuations that produce the farfield noise associated with the gear.
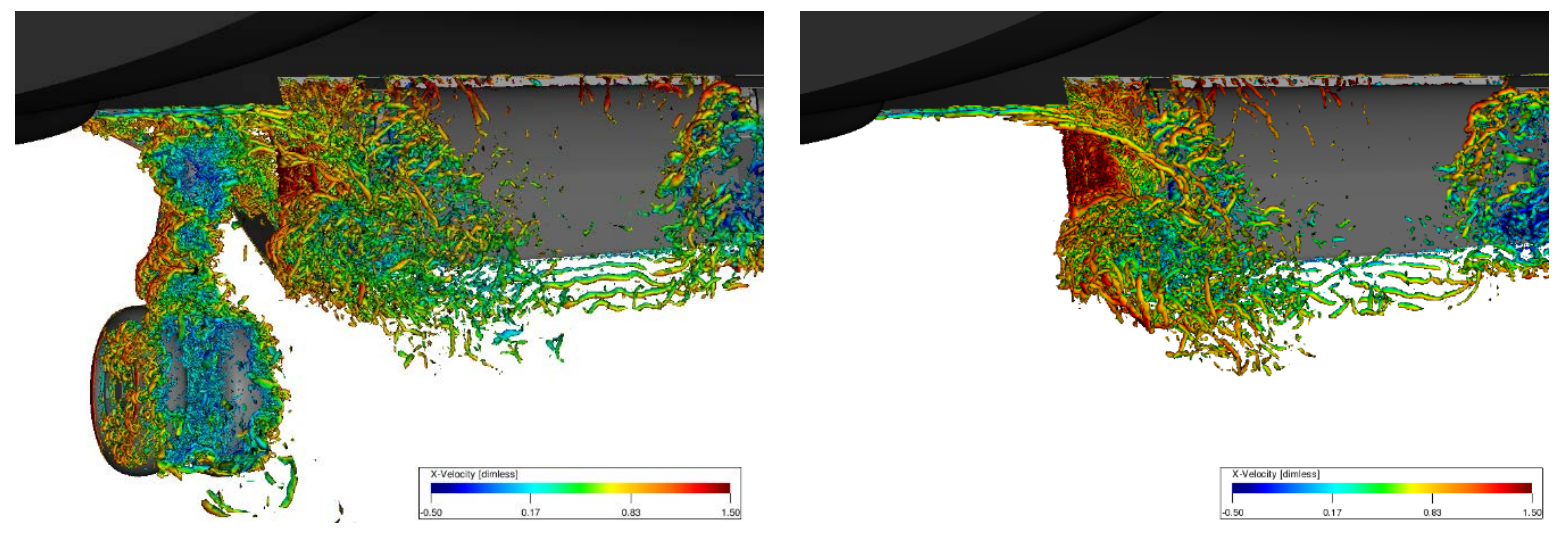

Figure 20. Close-up view of the gear-flap interaction zone showing instantaneous flow field based on isosurface of $\lambda_{2}$ criterion (at -3000) for gear on (left) and gear off (right) configurations.

The corresponding contour plots of the instantaneous and time-averaged flow field at the inboard edge are shown in Fig. 21. Observe from the figure that although the gear wake interacts with, and subsequently alters, the flow field at the inboard tip, a dual vortex system is maintained at the side edge. Compared to the gear off configuration (Fig. 17), the gear turbulent wake and its interaction with the emerging vortices are clearly seen in the unsteady flow field (left column). Also notice that with the gear on, merging of the two vortices at the inboard tip is accelerated so that a single dominant vortex is apparent at the flap trailing edge. 

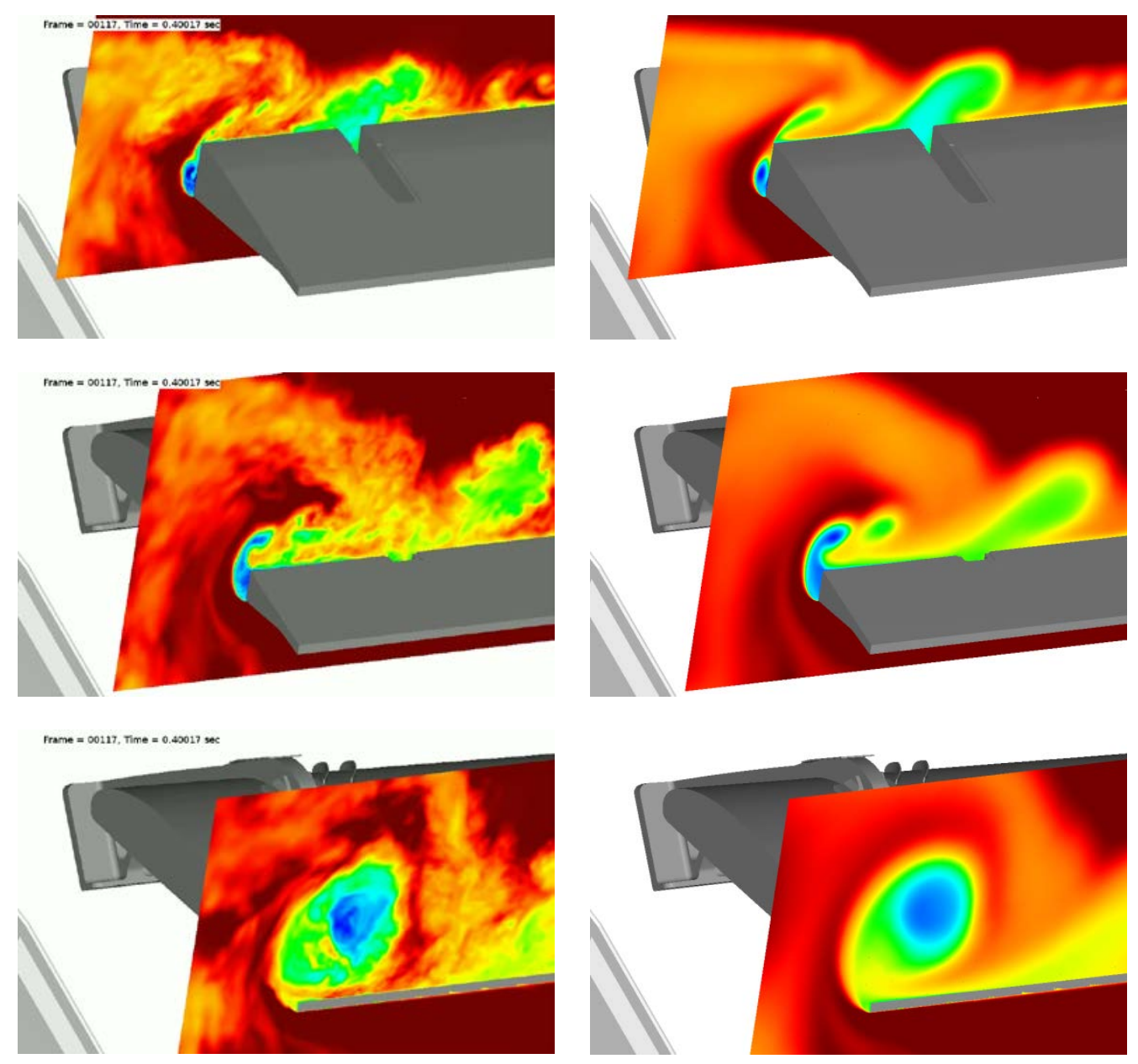

Figure 21. Total pressure contours of unsteady (left) and time averaged (right) flow at the inboard flap side edge for baseline configuration with main landing gear.

\section{Acoustic Field}

Considerable flow unsteadiness (noise sources) is produced during the shear layer roll-up, vortex formation, and vortex merging process, as well as by the interaction of the vortices with the sharp corners at the flap edge. A small portion of the energy associated with the pressure fluctuations (lift oscillations) is converted into sound waves that radiate outwardly away from the tips with a significant portion of the waves directed toward the ground. In addition, the presence of the cavity and the bulb seal at the outboard tip is expected to give rise to flow-cavity interaction and a possible cavity resonance within certain frequency bands. In general, because of the relative size of flow structures at the flap tips, the lower frequency portion of the radiating spectrum should be dominated by the noise sources residing at the inboard tip and the higher frequency range should be driven by the sources situated at the outboard tip. This conjecture was confirmed by the simulations and will be shown later when we consider the contribution of each tip to the total farfield noise spectrum.

A global view of the radiated sound field produced at the flap tips for the baseline configuration without the main gear is given in Fig. 22. The two-dimensional planar cut positioned at the flap mid-chord clearly shows the origin of the sound waves, which radiate spherically from both tips. The corresponding cut for the configuration with the main gear installed is presented in Fig. 23. Note from the figure that gear deployment does not diminish or significantly alter the sound levels nor the patterns associated with the inboard tip. However, as shown by the measurements presented in Ref. 11, deflection of the flap reduces the noise levels produced by the main landing gear. 


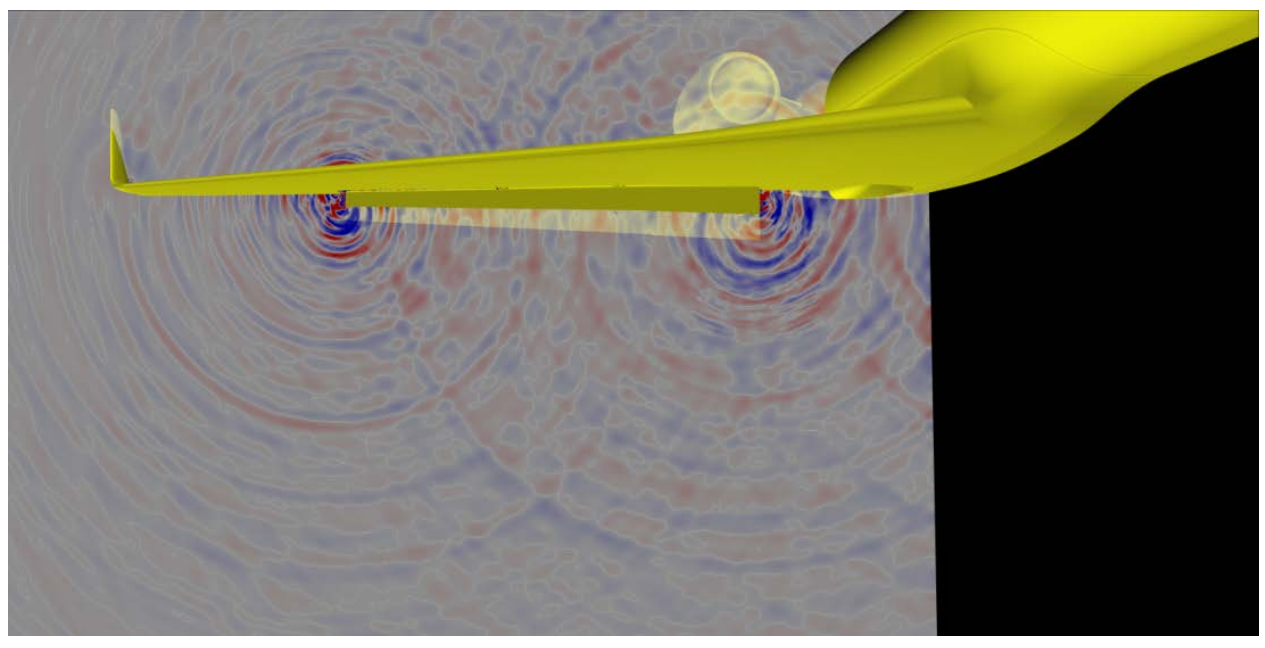

Figure 22. Radiated sound field (dilatation field) associated with noise sources at inboard and outboard flap tips (planar cut at flap mid-chord).

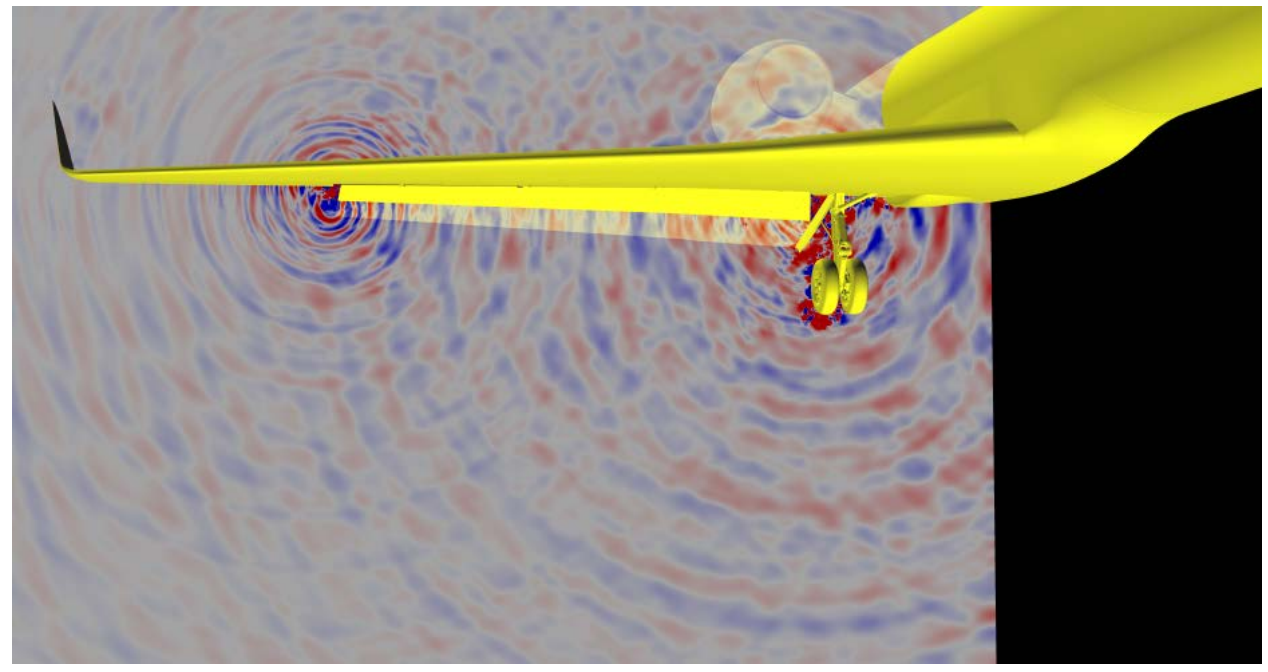

Figure 23. Radiated sound field (dilatation field) associated with baseline configuration with main landing gear deployed (planar cut at flap mid-chord).

\section{B. Surface Pressure Coefficient (Cp)}

The computed time-averaged Cp distributions for the model aircraft wing were found to be in good agreement with the measured values at all the pressure port rows. Sample Cp plots comparing computed and measured pressure fields at select rows on the wing and winglet are presented in Fig. 24 for the configuration without main landing gear. Results from both medium and fine resolution grids are displayed. Notice that nearly identical Cp values are produced by the two grids, indicating that a reasonable degree of grid independence has been attained for the magnitude of the steady lift produced by the wing. The computed Cp values display slightly higher suction peaks near the wing leading edge. Although not as pronounced, the over-prediction is maintained throughout to the wing trailing edge. In general, better agreement between computed and measured Cp is obtained for the pressure rows closer to the wing root than for the rows situated in the wing mid-span and outboard sections. Similar over-suction trends were also present in the companion computations of Khorrami and Mineck ${ }^{20}$ obtained using NASA's unstructured Navier-Stokes solver FUN3D in combination with a Modified Delayed Detached Eddy Simulation (MDDES) approach to simulate the flow field around the same model. The present PowerFLOW® wing Cp values are very similar to those reported in Ref. 20. This suggests that the underlying causes for the observed differences are not related to the flow solver nor to grid resolution issues, and reside elsewhere. There are three important 
sources that could produce such trends ${ }^{20}$. They relate to a) differences between open-jet tunnel and current free-field settings, b) model deformation (aeroelastic) effects, and c) wind tunnel floor boundary layer effects. For a discussion on these effects and their implications, see Ref. 20.

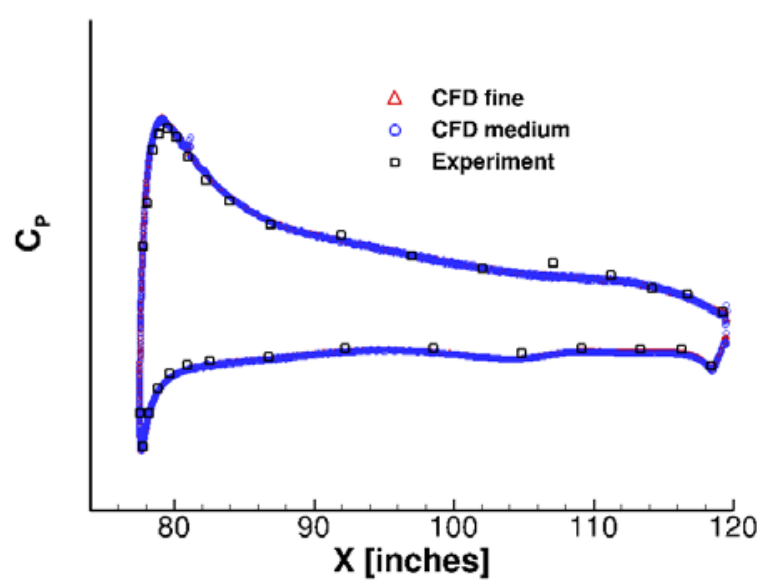

a) Wing row 1

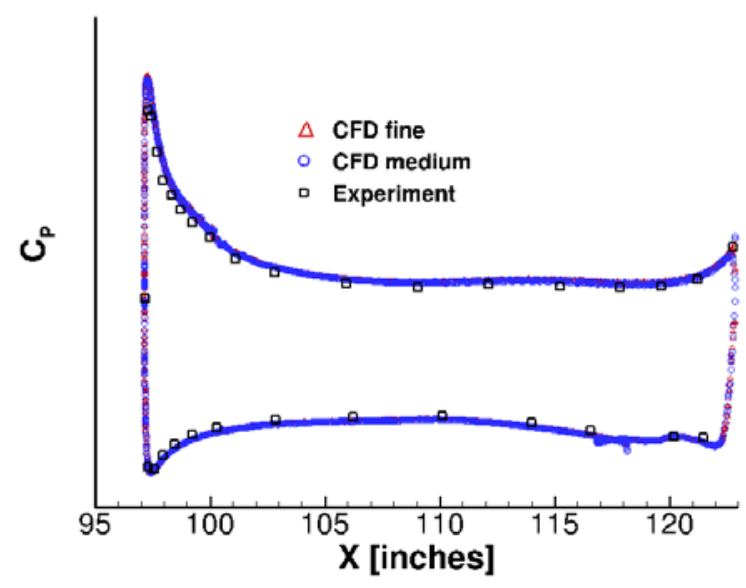

c) Wing row 5

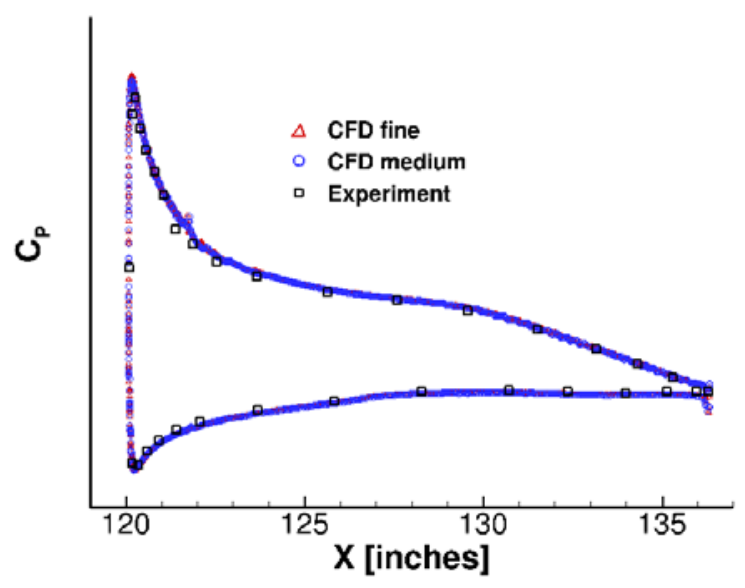

e) Wing row 9

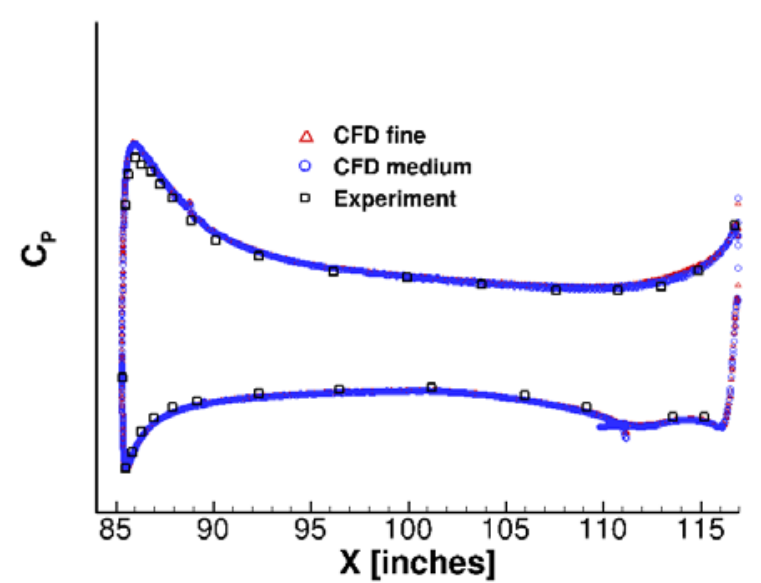

b) Wing row 3

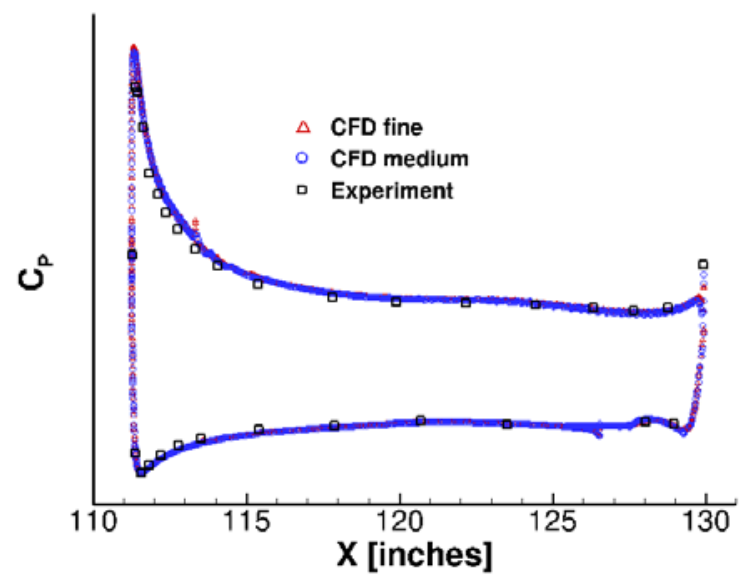

d) Wing row 7

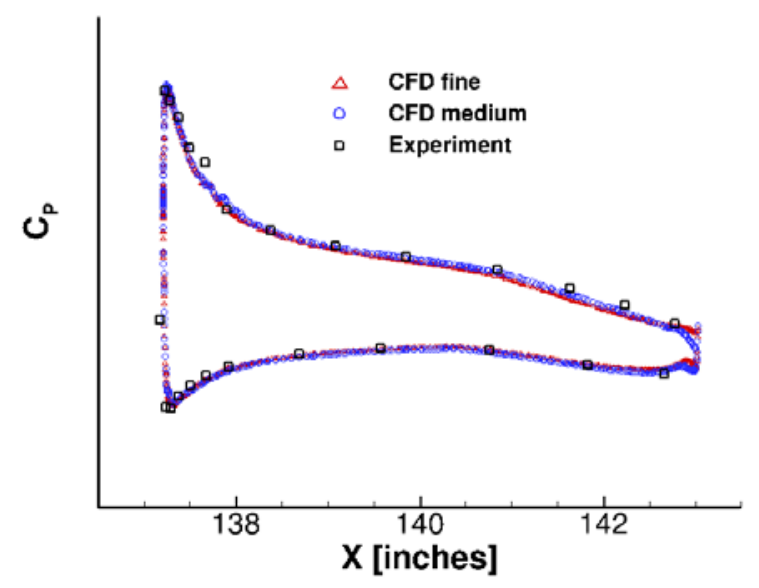

f) Winglet row 11

Figure 24. Surface pressures on wing and winglet. 
Time-averaged $\mathrm{Cp}$ values at select rows across the flap span for the baseline configuration without the main gear are displayed in Fig. 25. Except for a few localized minor improvements in accuracy achieved with increased mesh resolution, the computed pressures from the two grids are very similar. In general, good agreement between predicted and measured Cp values is observed at all sectional stations where pressure data are available (including those not shown here).

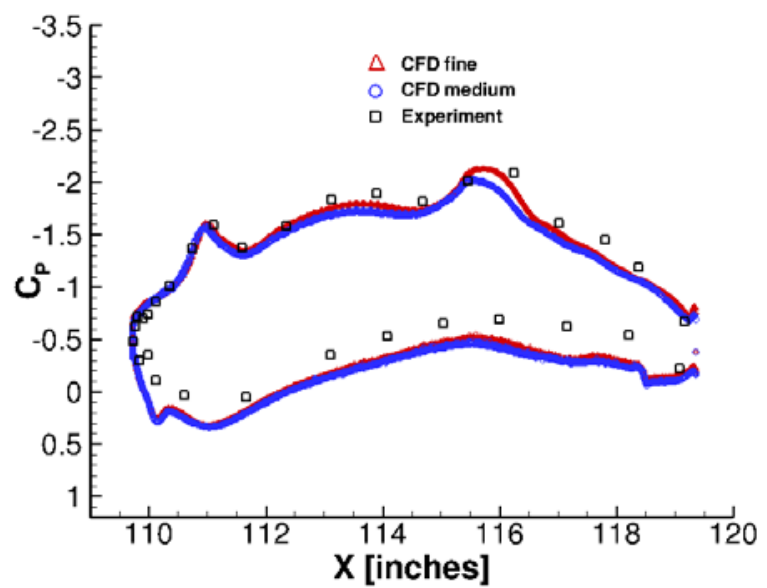

a) Flap row 1

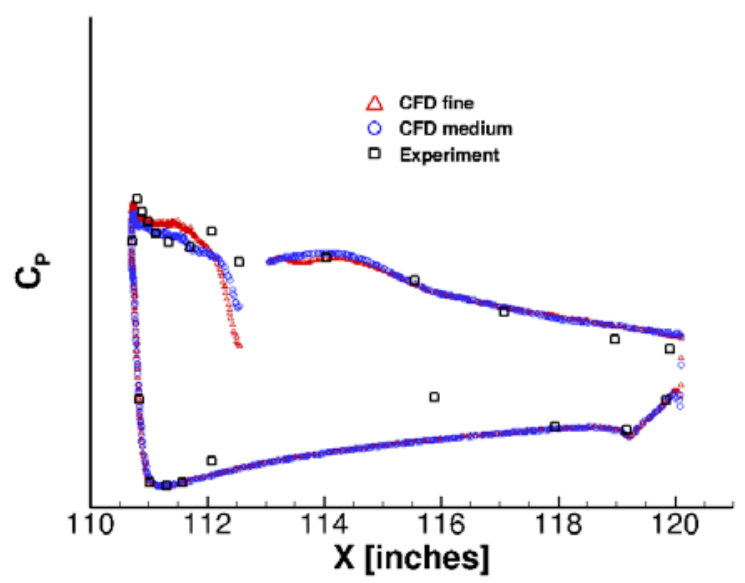

c) Flap row 3

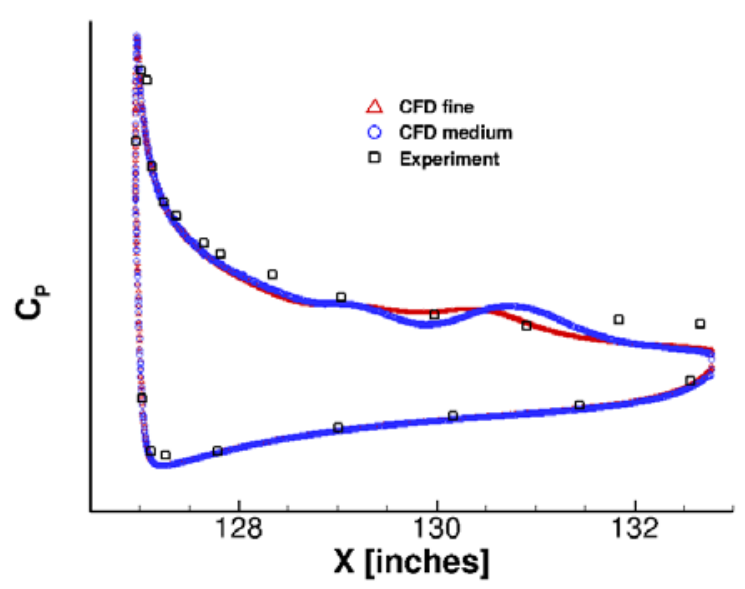

e) Flap row 9

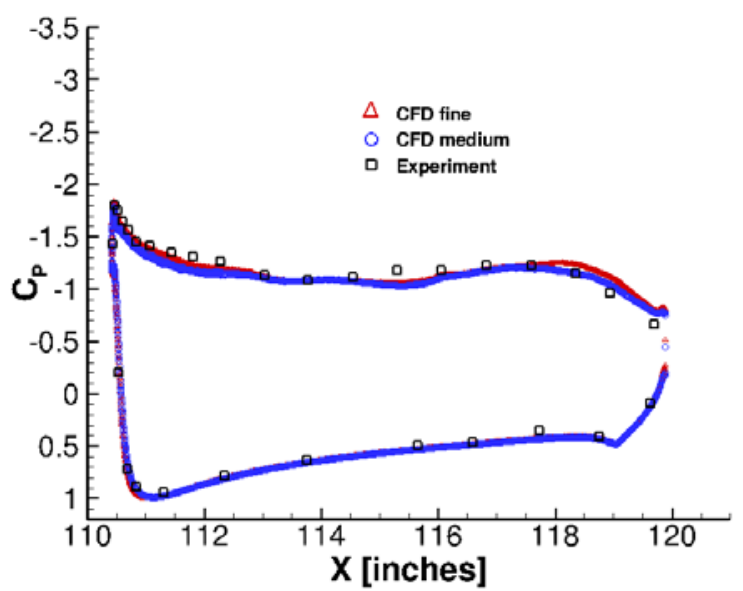

b) Flap row 2

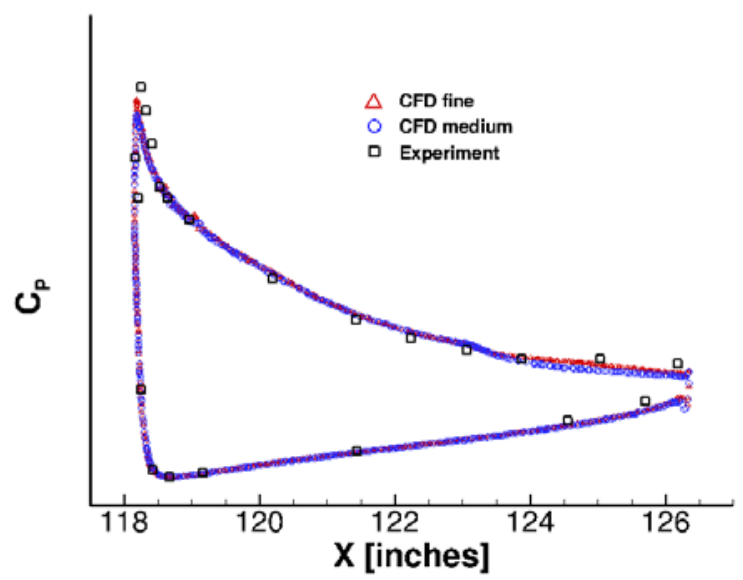

d) Flap row 6

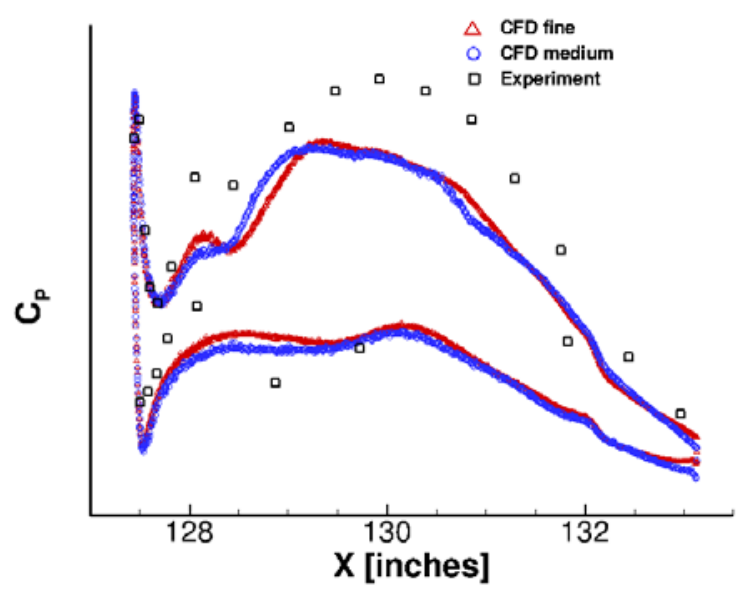

f) Flap row 11

Figure 25. Surface pressures on flap. 
Accurate simulation of the steady load at both inboard and outboard flap tips is of paramount importance to proper edge vortex formation, development, and migration, and therefore is critical to obtaining the corresponding fluctuating pressure field (noise sources). The averaged pressure distributions at the two closest rows to the flap inboard edge are plotted in Figs. 25a and 25b. The Cp values obtained from the medium and fine grid resolutions are in good agreement with the measured pressures. In the case of the pressure row closest to the inboard edge (row 1 ), increased resolution improved the accuracy of the predicted pressure field, enabling proper capture of the vortexinduced suction peak near the flap 0.6c $-0.7 \mathrm{c}$ region. However, both medium and fine resolution results show higher pressures on the flap bottom surface at the inboard tip. This is surprising since the bottom surface mostly experiences a skewed boundary layer flow rushing towards the edge, which is far less complex than the corresponding flow features that develop and reside at the top edge.

Good agreement between computed and measured Cp values is maintained past the flap mid-span (row 6). Beyond row 7, the measured surface pressures display the presence of a rather large flow separation zone that extends throughout the tip. The simulated Cp values at row 9 (Fig. 25e) associated with the medium resolution grid display an oscillatory behavior that starts at the mid-chord region and ends near the flap trailing edge. The amplitude of the oscillations diminishes noticeably with increased resolution, with both grids failing to predict the correct surface pressures beneath the separation zone for the last 35\% of the chord. We believe that this oscillatory feature in the time-averaged $\mathrm{Cp}$ values at rows 8 (not shown) and 9 is a remnant of low-frequency flow oscillations within the separation zone at these outboard locations; a substantially longer record is required for the time-averaging process to yield the proper steady values. The computed pressure distribution at the row closest to the outboard edge (row 11) is presented in Fig. 25f. At this row, although the overall character of the surface pressures was captured in the predictions, the simulated time-averaged $\mathrm{Cp}$ values substantially underestimate the broad suction region that occurs in the mid-chord region; the increased spatial resolution achieved with the fine grid mildly improves the comparison for the predicted values. A much larger under-prediction of the Cp values at row 11 is reported by Khorrami and Mineck ${ }^{20}$ who point to the possible shortcomings of the particular MDDES approach used in their study as the source of the observed discrepancies. The mid-chord suction region observed at the outboard tip is the footprint of the primary vortex that forms at the side edge (see Fig. 19). The lateral position of this vortex greatly depends on how well the steady spanwise flap loading near the outboard tip is captured, which in turn depends on an accurate prediction of the large flow separation zone. Note that most turbulence models cannot predict correctly three-dimensional boundary layer separation on smooth surfaces. Even more challenging are flows subjected to severely adverse pressure gradients and containing confluent boundary layers, which is the case for a highly deflected flap. Thus, it is encouraging that the present lattice Boltzmann approach seems to be predicting somewhat better pressure distributions, as compared to those provided in Ref. 20.

Deployment of the main landing gear only affects the time-averaged pressure field on the bottom surface of the wing (due to the gear cavity opening; row 3 in Fig. 2) and the region in the immediate vicinity of the flap inboard edge. Cp distributions for the two rows closest to the inboard edge (rows 1 and 2) are shown in Fig. 26. By the third flap row position (not shown), the differences in the surface pressure values for the configurations with and without the gear become negligible. Except for a localized, slight improvement in the prediction of upper surface pressures at row 1, the two grids produce nearly identical results. As was the case with the gear off configuration, good agreement with measurements is obtained except for the flap bottom surface at the inboard tip (in particular near the leading edge). The reasons for the over-prediction at this location are not clear to us. The favorable comparison displayed in Fig. 26 indicates that gear-flap interaction and its effect on the steady loading at the tip are well predicted. Our earlier observation that the wake of the gear is convected past the inboard portion of the flap, altering the tip loading, is confirmed by comparing the pressure coefficient plots shown in Figs. 25 and 26 for rows 1 and 2. Note that the row closest to the inboard flap side edge (row 1) exhibits an overall decrease in pressure difference between flap bottom and top surfaces, caused by the locally reduced inflow velocity within the gear wake. Also, observe that the location of vortex roll-up and coalescence on the suction side of the flap is shifted downstream by about $0.05 \mathrm{c}$. 


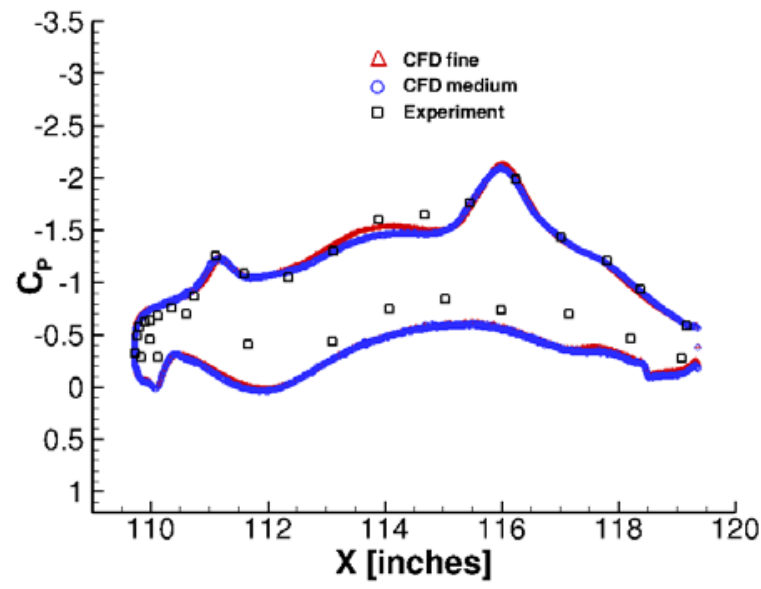

a) Flap row 1

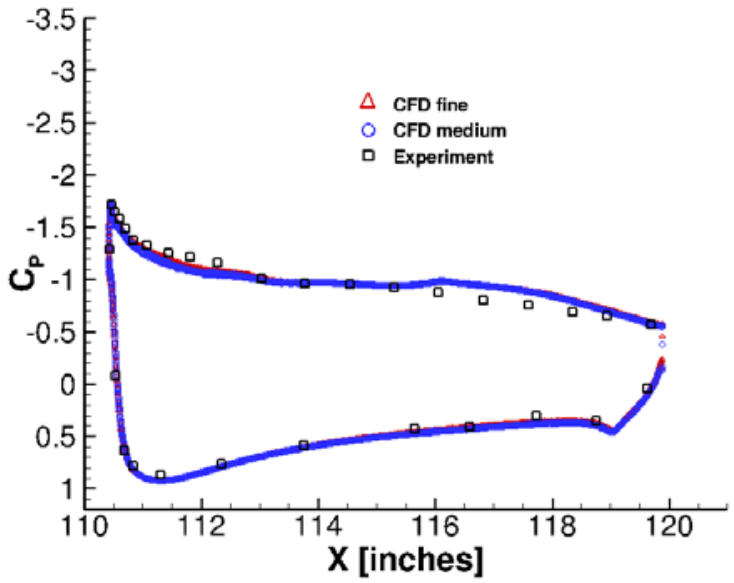

b) Flap row 2

Figure 26. Surface pressures at flap inboard edge for configuration with main gear on.

\section{Surface Pressure Fluctuations}

Accurate prediction of the surface pressure fluctuations in the vicinity of the flap side edges is critical for a proper assessment of airframe noise source location. Extensive comparisons between computed and measured fluctuating pressure fields for all dynamic probe locations revealed remarkable agreement at most measurement positions, with some probes displaying slightly better, and some others showing slightly worse, agreement than the power spectral density (PSD) plots presented next.

\section{1. $\quad$ Fluctuations at Inboard Tip}

The simulated PSD values at four select probes placed along the edge on the flap top surface are presented in Fig. 27. As can be seen, medium and fine resolution grids produce very similar spectra at every probe location, with both solutions predicting correctly the measured spectrum in shape, frequency content, and energy levels. Closer to the flap leading edge (probe 67), where the flow features are expected to be minute, the simulated pressures are in remarkably good agreement with the measured data. The pressure spectra from representative locations (probes 4 and 9) on the top surface away from the edge are shown in Fig. 28. These sensors are part of a larger collection of probes specifically placed to capture the post-merged tip vortex footprint on the flap top surface. At these surface locations, the computed and measured pressures agree quite well, especially at probe location 9, where very good agreement is obtained for frequencies up to $20 \mathrm{kHz}$. Also observe that the medium-density grid provides sufficient resolution in the aft section of the flap chord, which is a pleasant surprise since the tip vortex path at that location has already detached from the flap top surface.

The pressure spectra from two of the probes (10 and 12) installed on the inboard edge-side wall are plotted in Fig. 29. Notice that the mild, broad tonal hump $(400-1,200 \mathrm{~Hz})$ present in the measured spectrum at the location of probe 10 is well matched by the simulations. The good agreement obtained at these two probes indicates that the sequence of fluid dynamic processes necessary for vortex inception at the edge was resolved appropriately.

The fluctuating pressure field at two probe locations (probes 15 and 16) on the bottom surface of the flap is presented in Fig. 30. As was the case for most other probe locations sampled, good agreement between computed and measured PSD values is observed. As expected, the fluctuation energy levels on the bottom surface are significantly lower than those recorded on the side wall or on the top surface. The close agreement observed for probe 16 in the low to mid frequency range is surprising, since this probe is positioned inward from the bottom edge (see Fig. 4b). This probe is mostly exposed to the passage of a highly skewed accelerating boundary layer drawn to the edge by the presence of a large pressure suction region induced by the tip vortex. The most logical explanation for the good agreement seen in Fig. 30b would be that the boundary layer on the flap bottom surface was laminar during the test, and the fluctuating pressure field recorded in this area is the imprint of the radiating pressure waves produced at the inboard tip. The underlying source of the rise in the measured spectra for probe 16 beyond 5-6 kHz is unknown. The increased levels could be caused by hydrodynamic pressure fluctuations associated with the boundary layer on the bottom surface, or by self-noise resulting from the pinhole installation of the dynamic sensors. 


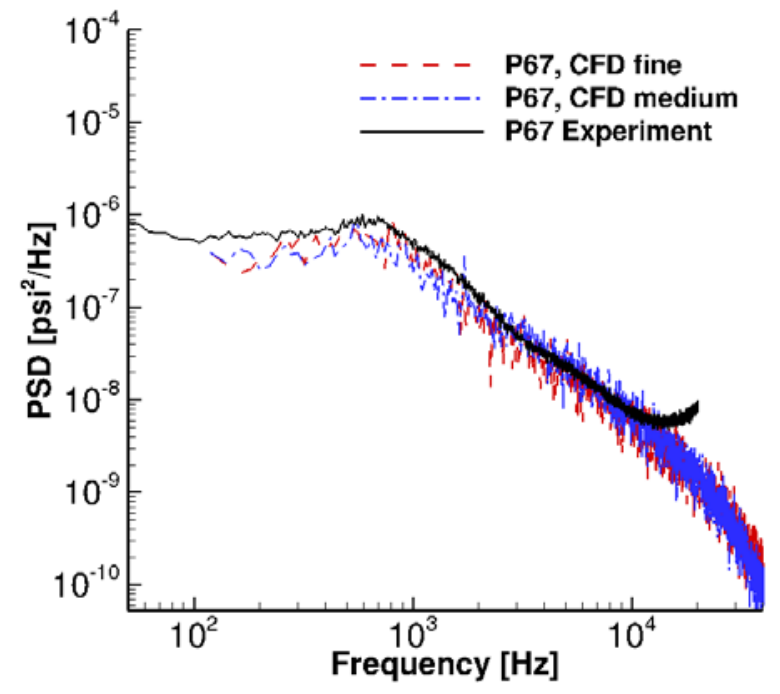

a) Probe 67 location

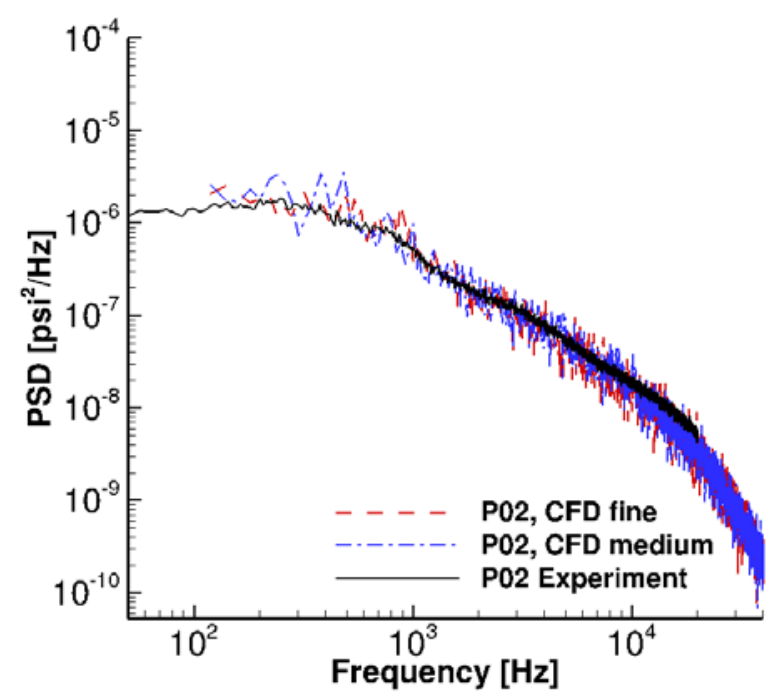

c) Probe 2 location

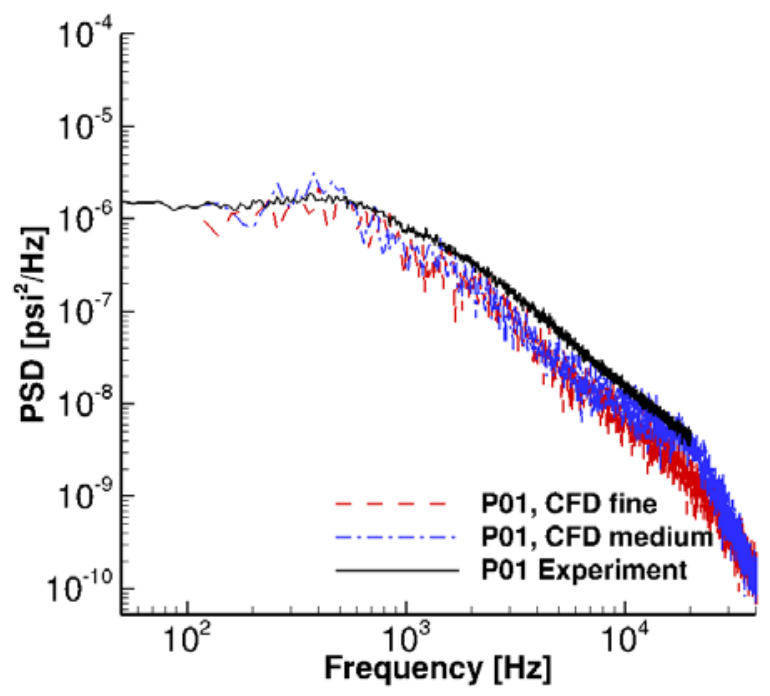

b) Probe 1 location

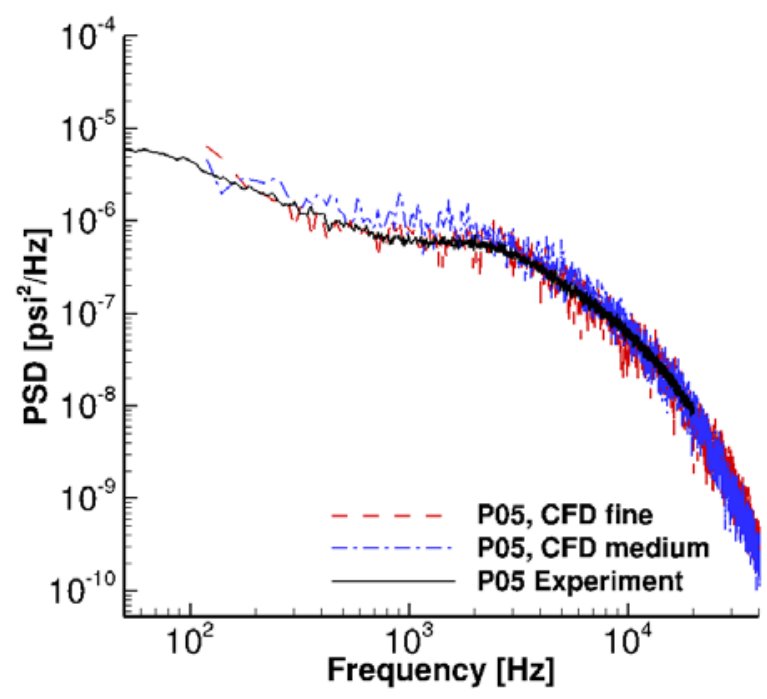

d) Probe 5 location

Figure 27. Power spectral density of surface pressure fluctuations at flap inboard tip - top surface edge. 


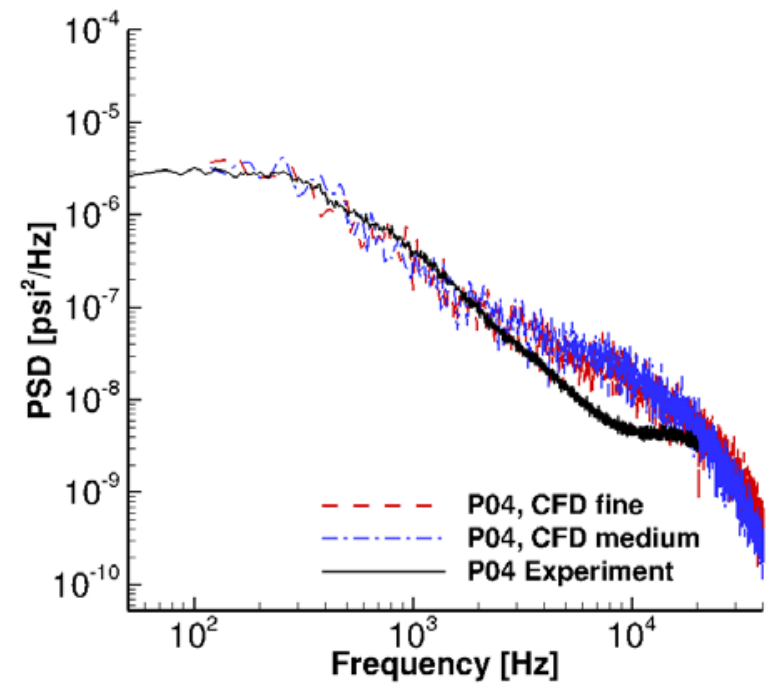

a) Probe 4 location

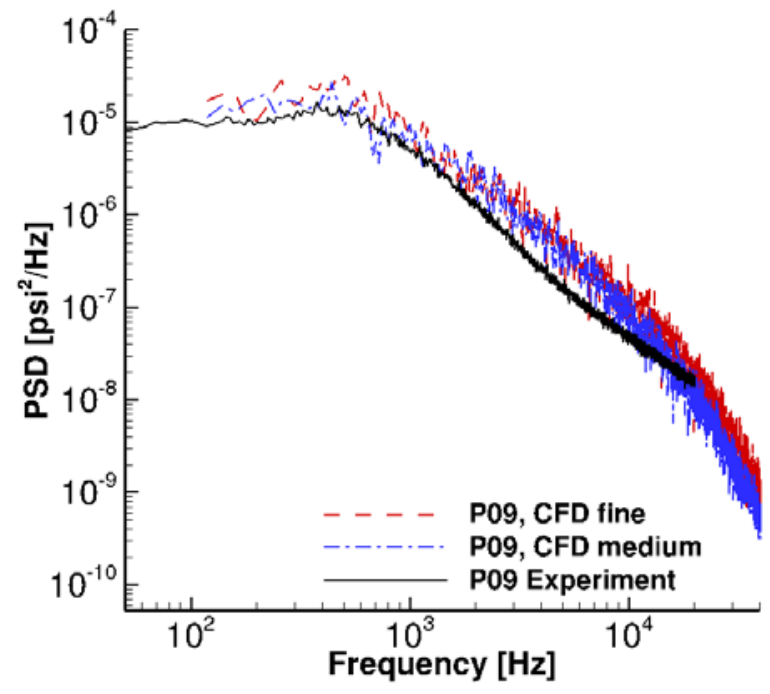

b) Probe 9 location

Figure 28. Power spectral density of surface pressure fluctuations at flap inboard tip - top surface away from edge.

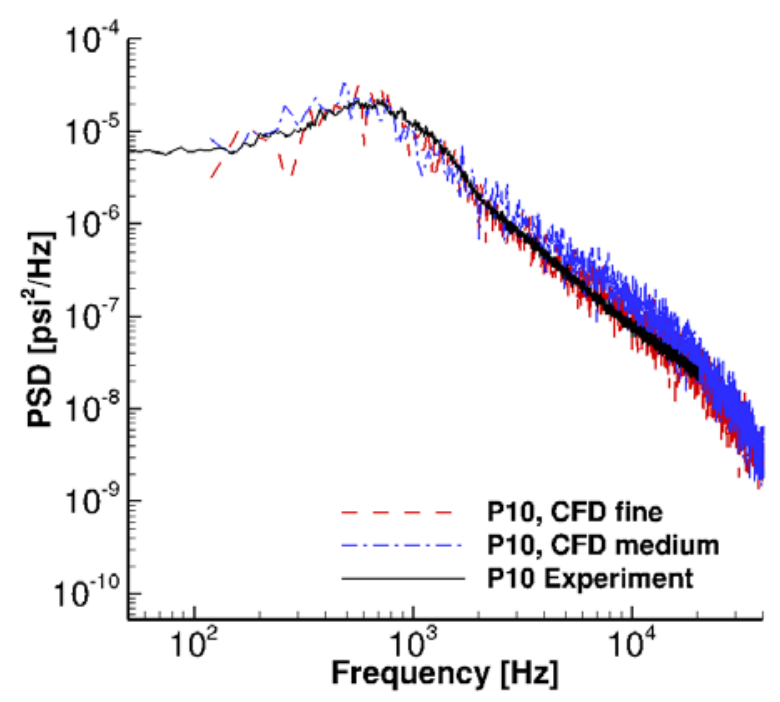

a) Probe 10 location

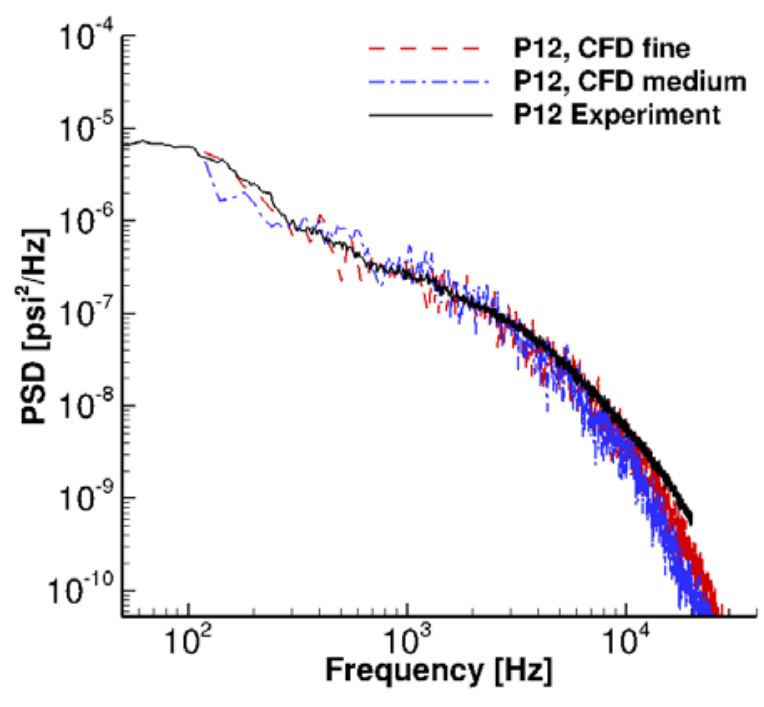

b) Probe 12 location

Figure 29. Power spectral density of surface pressure fluctuations at flap inboard tip - side-edge wall. 


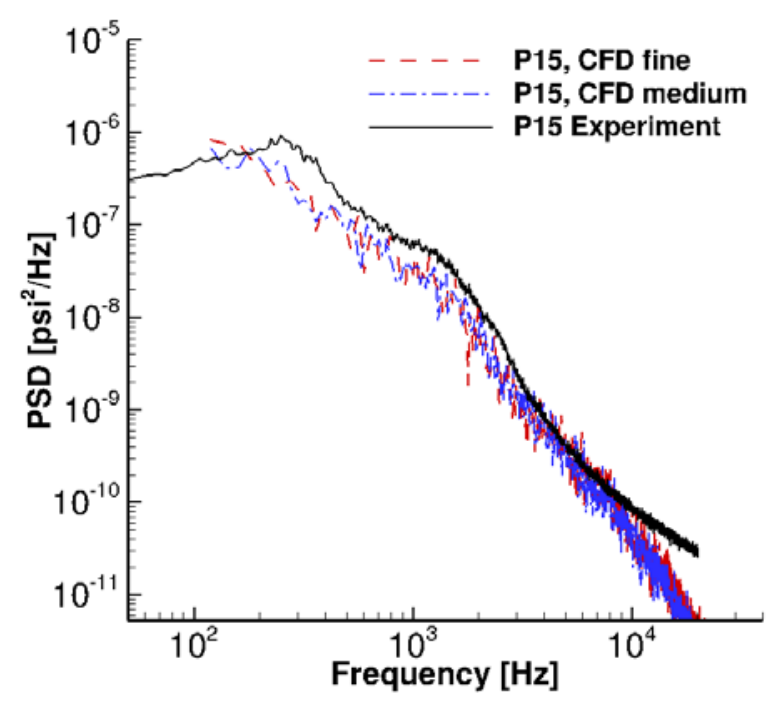

a) Probe 15 location

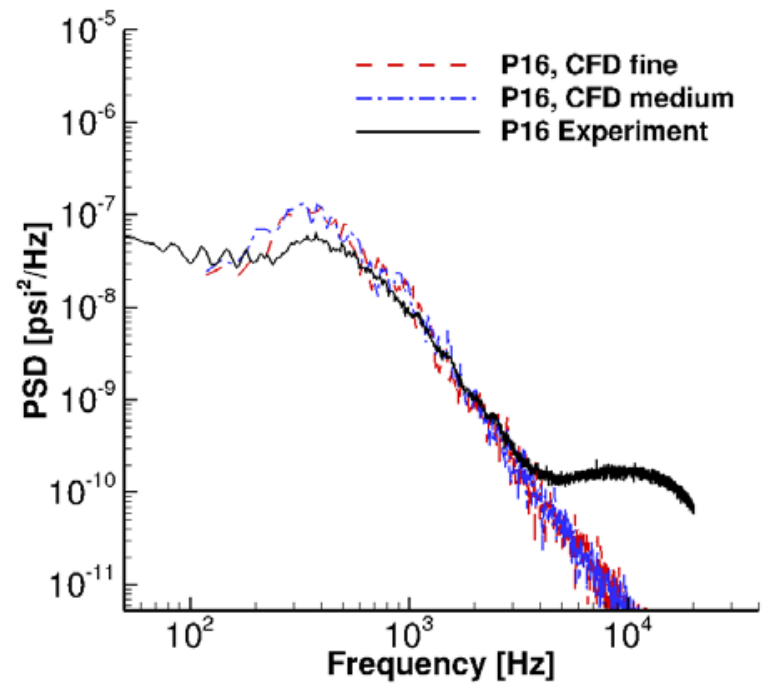

b) Probe 16 location

Figure 30. Power spectral density of surface pressure fluctuations at flap inboard tip - bottom surface.

\section{2. $\quad$ Fluctuations at Outboard Tip}

Prediction of the surface pressure fluctuations at the outboard tip was complicated by the difficulty in producing a realistic geometry of the bulb seal at the outboard tip that closely adhered to what was actually tested. In the $14 \times 22$ wind tunnel tests, the tubular soft foam used to represent the bulb seal was hand glued to the lower interior surface of the cavity at the tip with roughly a third of the tube residing exterior to the cavity; thus, there is a level of uncertainty in correctly duplicating the position of the tubular foam in the simulated model geometry. During testing, the soft foam may have deformed slightly under aerodynamic loading; in contrast, the modeled tubular foam in the simulations was treated as a rigid cylinder. Due to the installed pressure tubes and sensors, the as built/tested tip cavity has many protrusions and depressions on its interior surfaces that are absent in the simulated geometry. Because of these subtle differences, accurately matching the surface pressure fluctuations at discrete locations near the outboard tip is problematic at best. The critical parameter used to judge the success of the simulations is how well the farfield acoustic behavior is predicted. As will be shown in the following section, very good agreement between the simulated and measured farfield noise spectra was achieved.

Pressure fluctuations from selected probes along the edge on the flap top surface are presented in Fig. 31. In general, very good agreement between computed and measured spectra was attained along the upper edge. Unlike the pressures at the inboard tip (see Fig. 27), the spectra associated with the outboard edge contain several mild, broad tonal features that result from vortex-cavity interaction. In general, increased spatial resolution improves the predicted results. However, for probes 65 and 25, the better resolved pressure field displays mildly overpredicted energy levels for fluctuations within the $2-5 \mathrm{kHz}$ range. Since results from the two grids are in close agreement at higher and lower frequency bands, the reason for this local increase is not yet clear to us and requires further investigation. At the location of probe 28, the added spatial resolution eliminates the overprediction in the fluctuating energy levels - the fine grid spectrum is almost coincident with the measured curve, closely matching the localized tonal character of the spectrum. Farther downstream, at probe 66, the tonal peak near $2 \mathrm{kHz}$ and the sharp roll-off in the spectrum immediately after are well predicted.

Pressure spectra from probes 29 and 30, situated on the top surface away from the edge, are shown in Fig. 32. As was the case for the inboard tip, these sensor positions were selected to extract the post-merged footprint of the tip vortex on the flap upper surface. At probe 29 location, the simulated PSDs are in very good agreement with the measured spectrum. At probe 30, which is downstream of probe 29 and farther away for the edge, the PSD levels are overpredicted by both grids although the results for the finer grid are closer to the measured values over the entire frequency range. The computed spectra from the two probes inside the tip cavity (probes 26 and 27) are presented in Fig. 33. At the location of probe 26 , that is, close to the flap leading edge, the simulated spectra compare well with measurements. Increased spatial resolution improved the agreement for frequencies above $4 \mathrm{kHz}$. As was the case for the simulated PSD at probes 65 and 25, the better resolved spectrum displays a marked increase in the pressure fluctuation intensity levels within the $200 \mathrm{~Hz}$ to $4 \mathrm{kHz}$ frequency range. As noted earlier, the cause of this 
discrepancy is unknown at the moment. However, observe that this anomaly only occurs at probes located at the outboard tip near the flap leading edge region. Good agreement between computed and measured spectra was obtained for the second probe in the cavity (probe 27), which is located at about 0.60c from the leading edge. Notice that the computed spectra contain a tonal feature centered around $6.5 \mathrm{kHz}$, where a mild break in the slope of the measured data is observed; nevertheless, the more pronounced tonal feature centered around 1,200 $\mathrm{Hz}$ is missed by both simulations. Spectra obtained from probes on the flap bottom surface (probes 32 through 35) are shown in Fig. 34. For this surface as well, the simulated results are in remarkably good agreement with the measured data. Probe 32, which is situated near the leading edge, displays overpredicted levels with grid refinement. In general, the fluctuation levels on the bottom surface are one to two orders of magnitude lower than those observed on the top surface.

Overall, the pressure spectra predicted with PowerFLOW ${ }^{\circledR}$ at both inboard and outboard flap tips are in remarkably good agreement with the measured PSDs for the baseline configuration without the main landing gear. Since surface pressure fluctuations are essential to any noise prediction methodology, the close agreement obtained with these simulations is auspicious for accurate farfield noise computations.

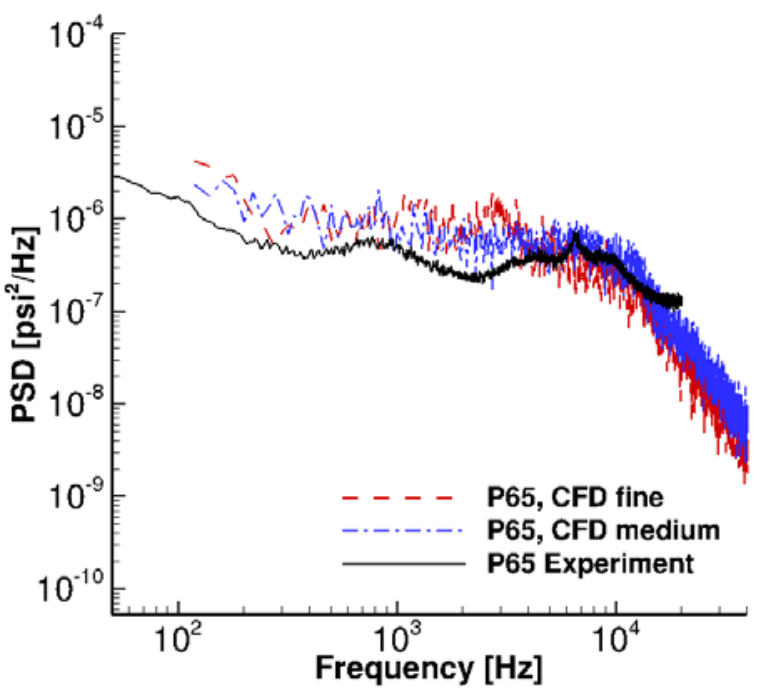

a) Probe 65 location

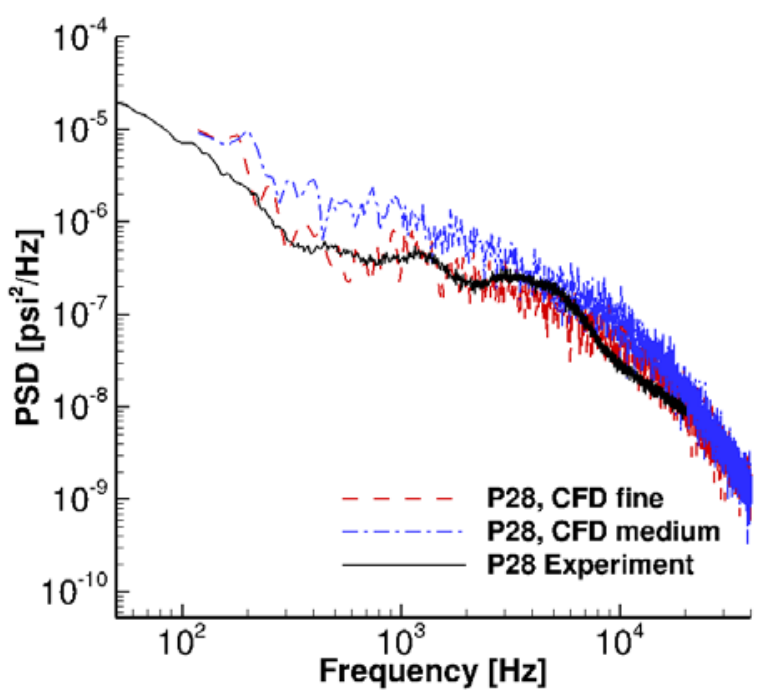

c) Probe 28 location

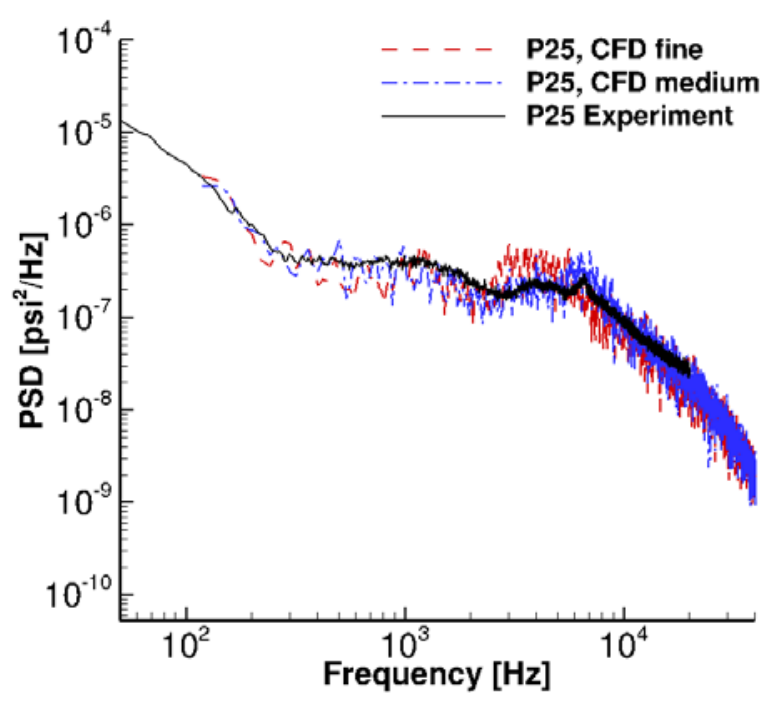

b) Probe 25 location

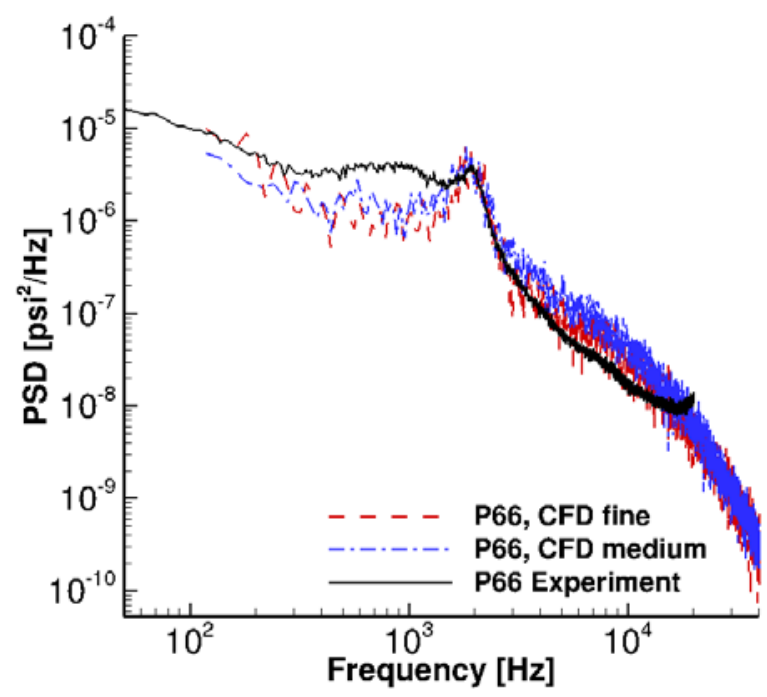

d) Probe 66 location

Figure 31. Power spectral density of surface pressure fluctuations at flap outboard tip - top surface edge. 


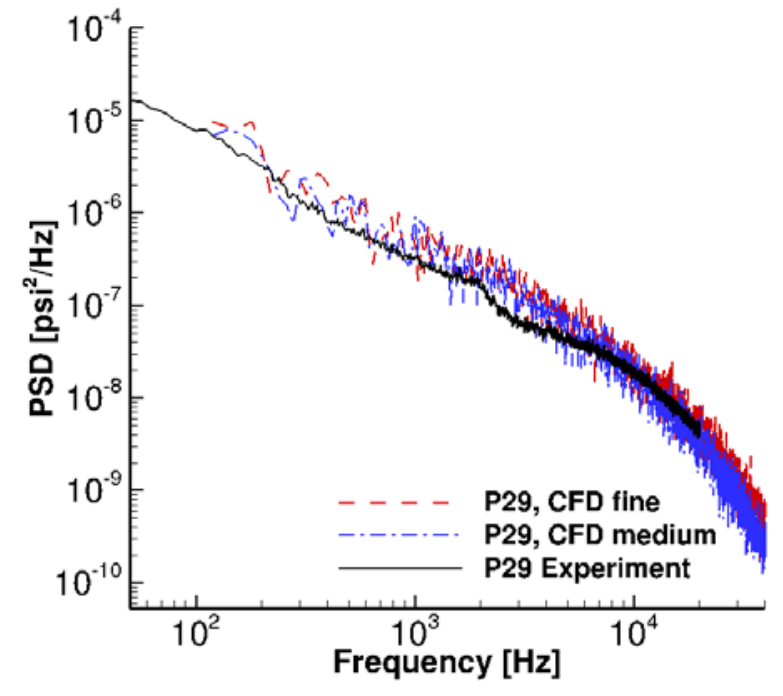

a) Probe 29 location

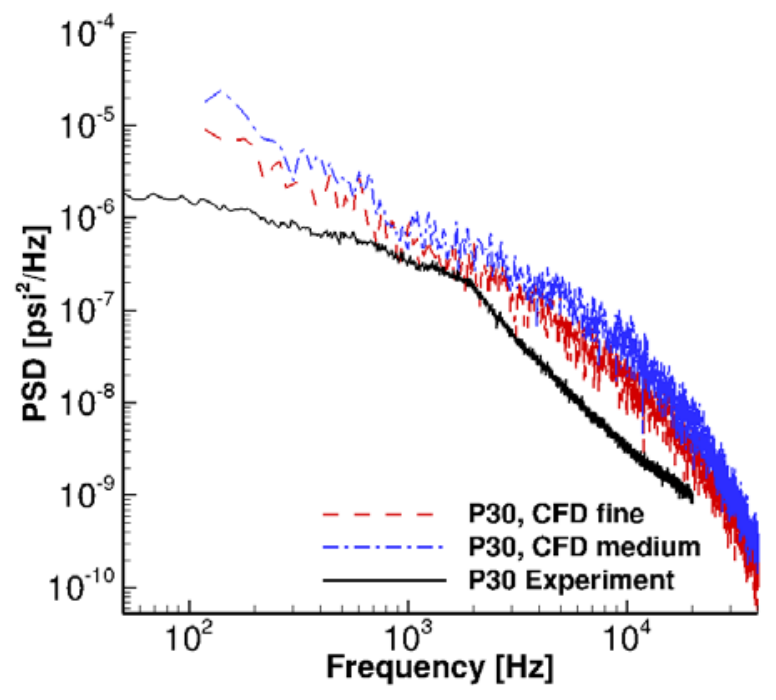

b) Probe 30 location

Figure 32. Power spectral density of surface pressure fluctuations at flap outboard tip - top surface away from edge.

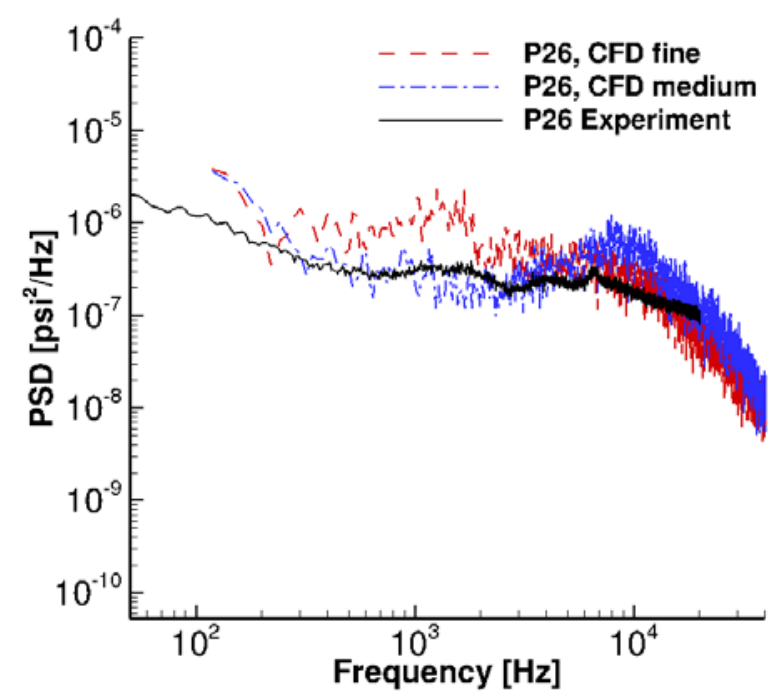

a) Probe 26 location

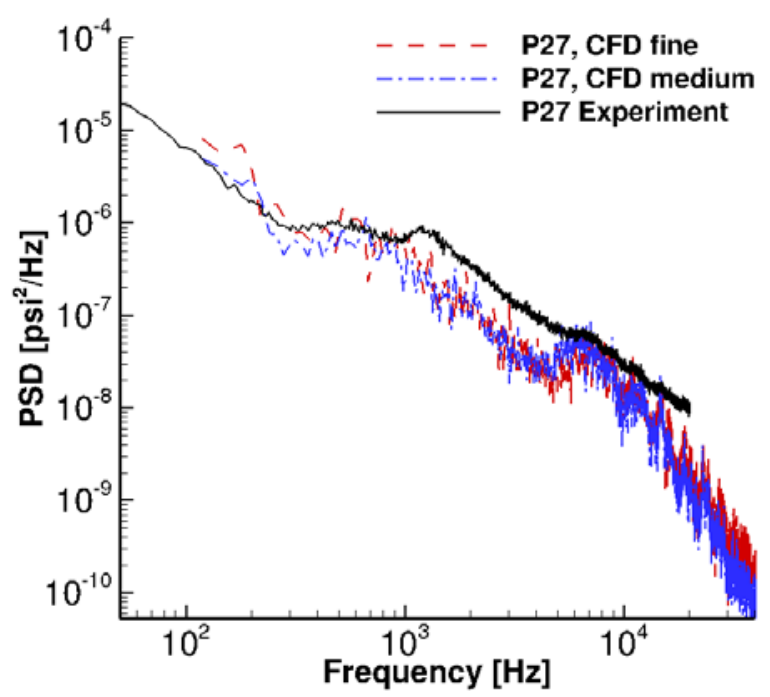

b) Probe 27 location

Figure 33. Power spectral density of surface pressure fluctuations at flap outboard tip - cavity wall. 


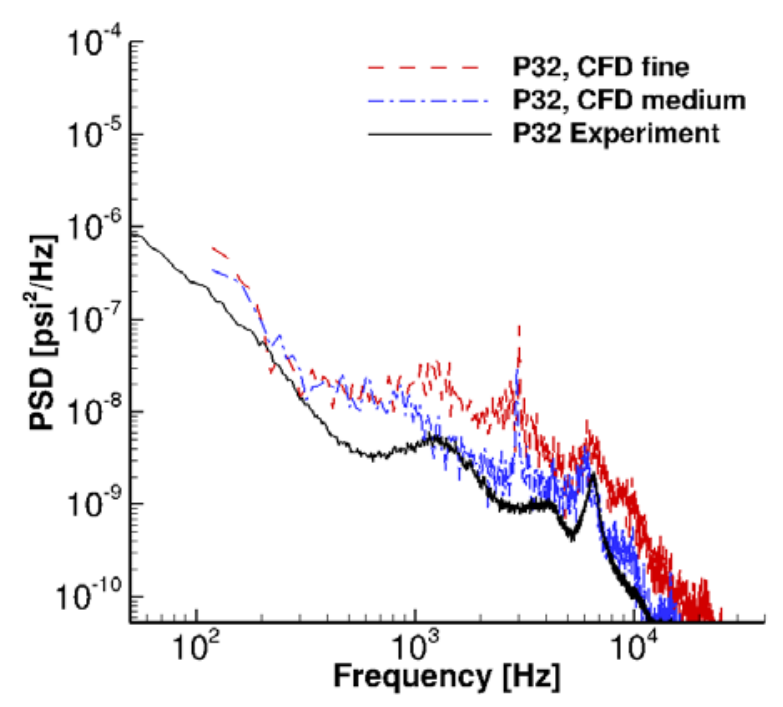

a) Probe 32 location

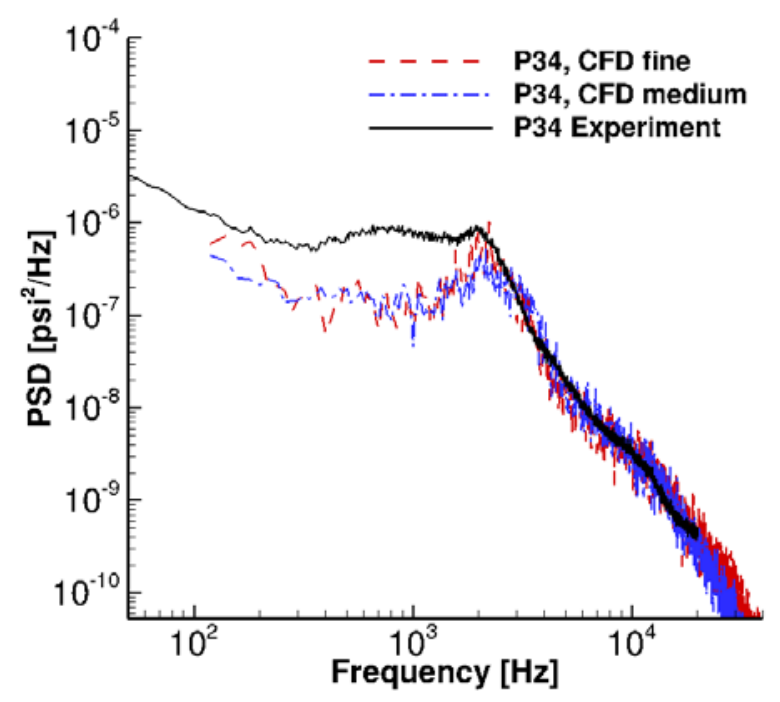

c) Probe 34 location

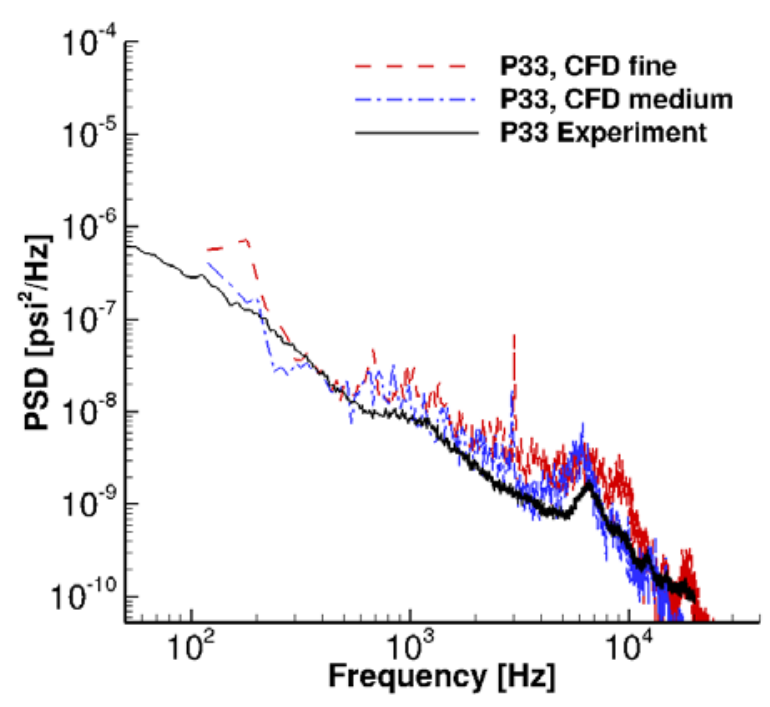

b) Probe 33 location

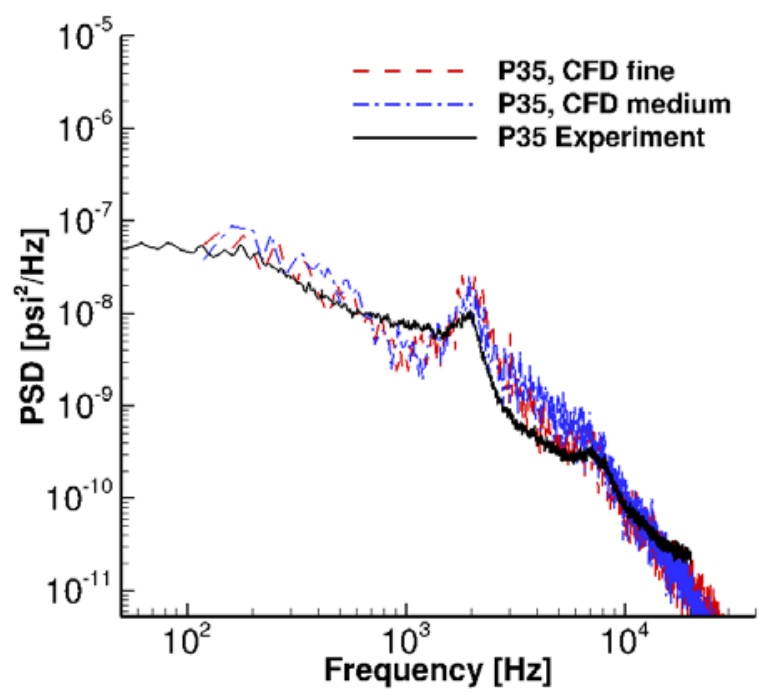

d) Probe 35 location

Figure 34. Power spectral density of surface pressure fluctuations at flap inboard tip - bottom surface.

\section{Main Landing Gear}

The gear-flap interaction as it affects the fluctuating pressure field at the flap inboard tip is examined in this section. We will probe a select number of locations where noticeable changes in the measured values were shown to occur in Ref. 10. The PSD comparison at various probe locations on the upper surface and side wall at the flap inboard tip are presented in Fig. 35. The predicted spectra are in excellent agreement with the measurements on the top surface and side-edge wall for the two grid resolutions shown. As observed from the experimental spectra, gear deployment noticeably increases the magnitude of the fluctuation levels at probes $1,2,4$, and 12 . The simulations capture this rise and the change in spectral shapes remarkably well. Although not shown, good agreement was also observed at other inboard probes. The predicted and measured spectra for the outboard tip were unaffected by the presence of the gear; therefore, they are not included here. 


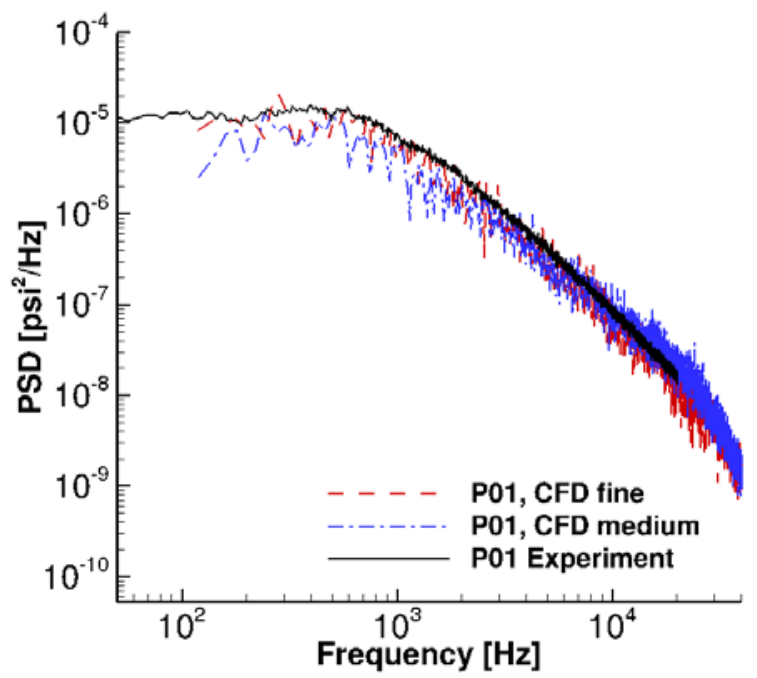

a) Probe 1 location

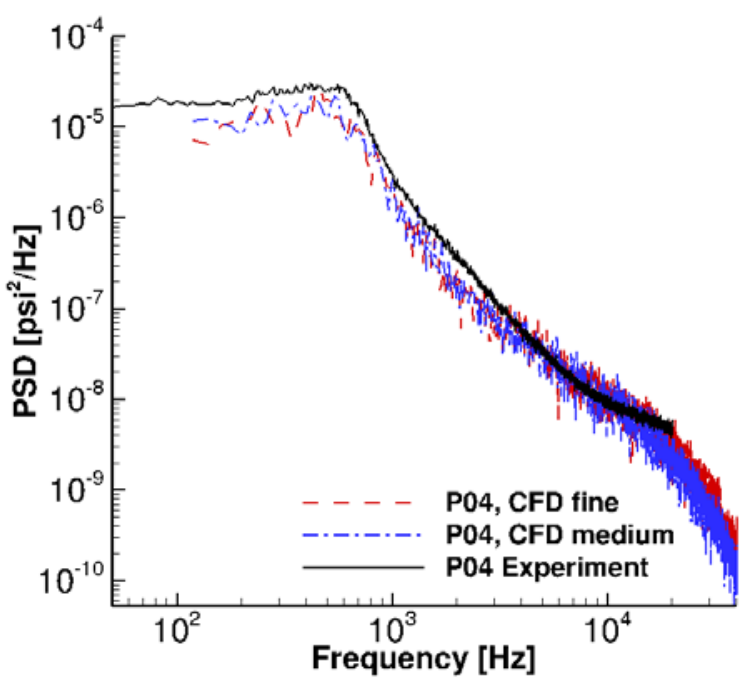

c) Probe 4 location

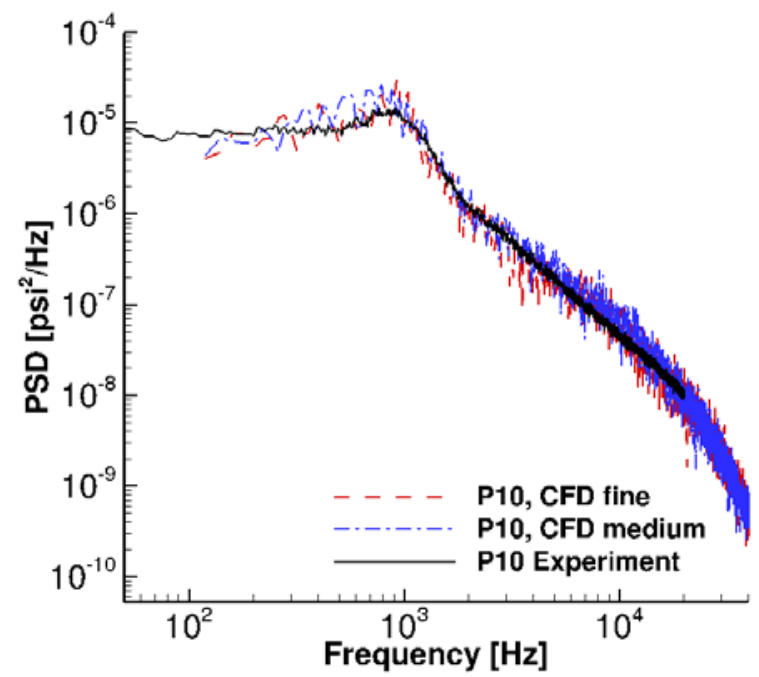

e) Probe 10 location

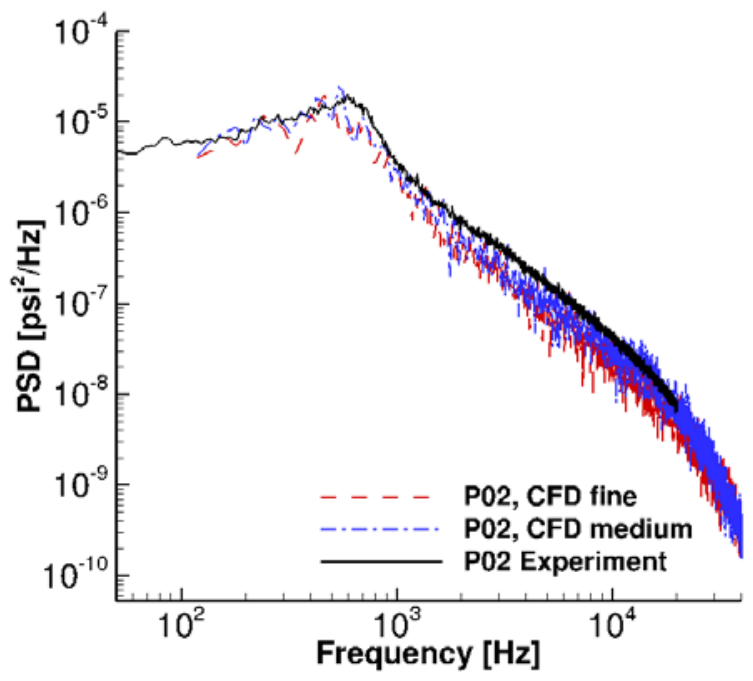

b) Probe 2 location

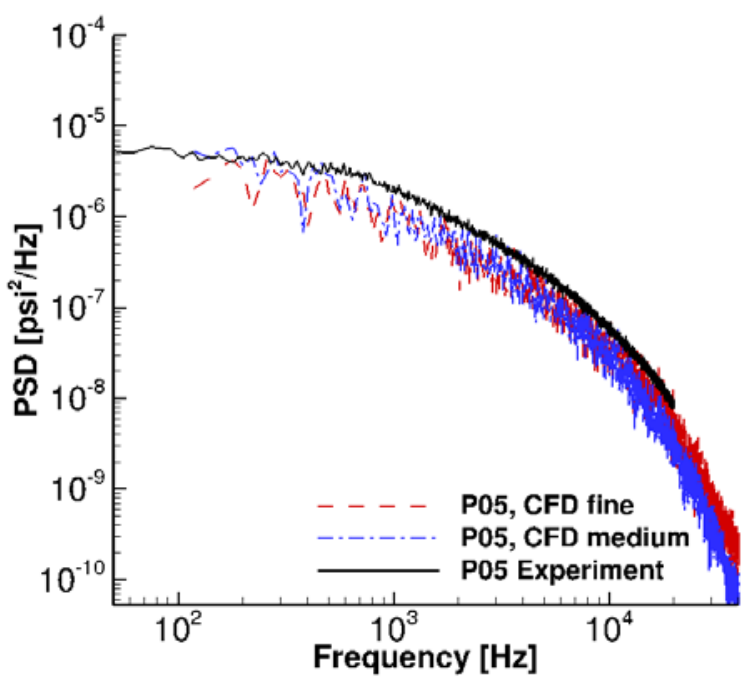

d) Probe 5 location

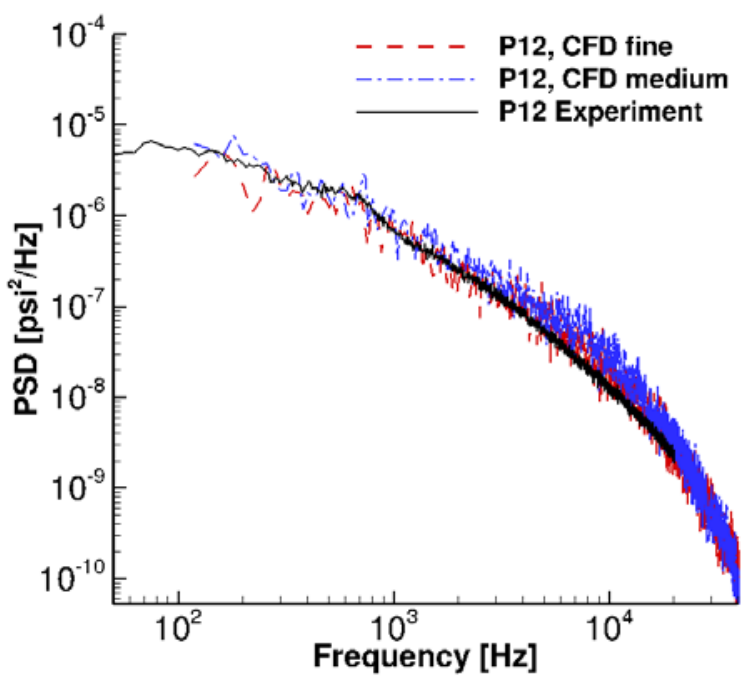

f) Probe 12 location

Figure 35. Power spectral density of surface pressure fluctuations at flap inboard tip with main gear on. 
Because of the bluff body nature of the various gear subcomponents, prediction of the measured surface pressure spectra at discrete locations is problematic at best. For example, the presence of a slightest flow angularity in the tunnel freestream velocity, and/or the differences between the free-field computational setup and the open-jet wind tunnel tests, have the potential to cause a significant shift in the location of flow separation and reattachment points. Computed surface pressure spectra at select locations on the gear primary subcomponents (struts, wheels, and door) are presented next.

Measured and simulated surface pressure spectra for probes located on the front (36 and 37, see Fig. 8) and rear struts (39 and 40) are shown in Fig. 36. These probes were placed at strategic positions to capture flow separation from the gear main post and the impact of the free shear layer on the rear strut.

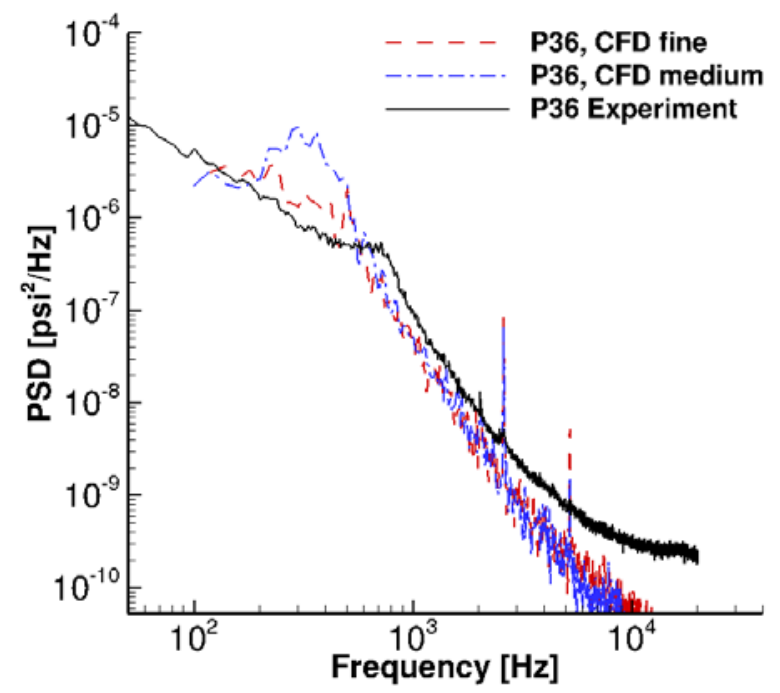

a) Probe 36 location

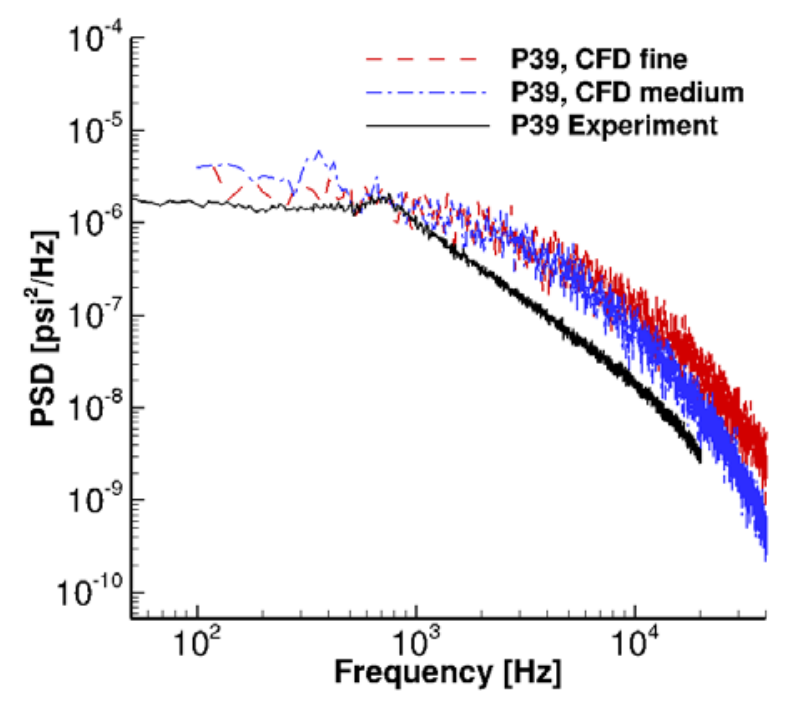

c) Probe 39 location

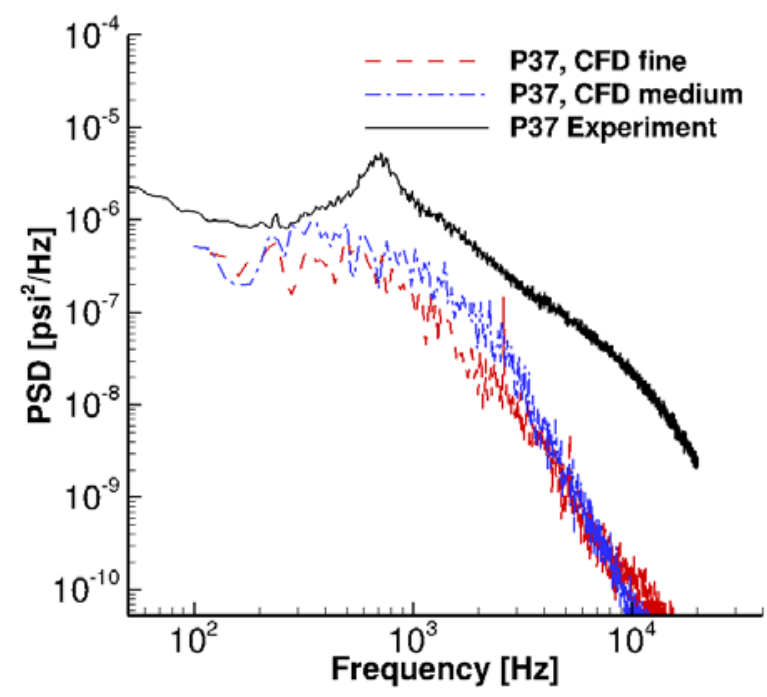

b) Probe 37 location

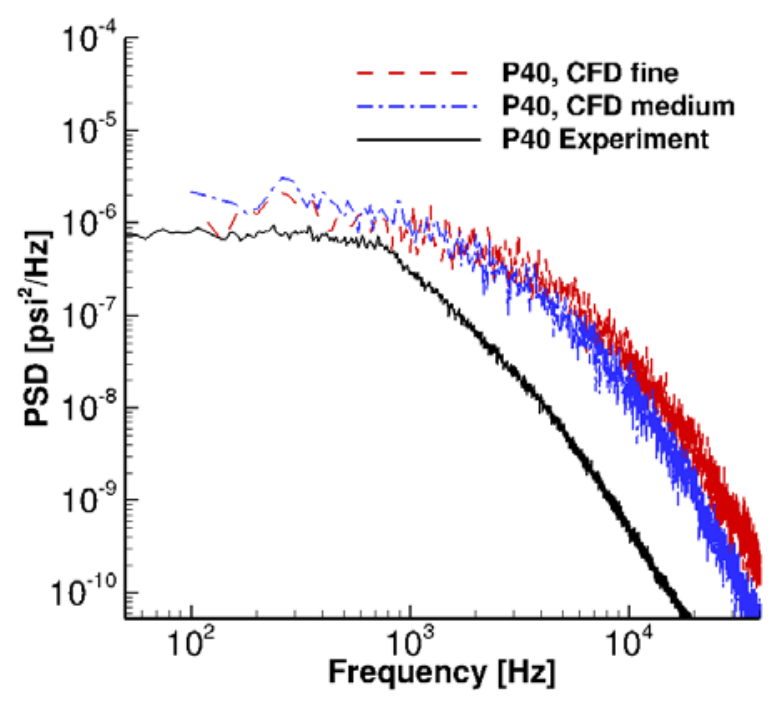

d) Probe 40 location

Figure 36. Power spectral density of surface pressure fluctuations on main gear front (a) and (b), and rear (c) and (d) struts.

Except for the PSD values associated with probe 36, the computed spectra reveal mixed trends. The measured spectrum at probe 37 has a broad tonal feature with a peak frequency around $700 \mathrm{~Hz}$. Based on the physical dimensions of the main post, most likely this tone represents mild vortex shedding from the post. The spectrum predicted at the location of probe 37 significantly under-estimates the energy level at all frequencies and fails to capture the tonal component of the measured data. The computed results for this probe are remarkably similar to the 
FUN3D predicted PSD values reported in Ref. 20. This suggests that there is a substantial difference between the two independent simulations and the measurements regarding the location of boundary layer separation on the main post, possibly associated with the laminar to turbulent transitional behavior of the flow on the cylindrical struts, which is not taken into account in the simulation. An opposite trend is observed for probes 39 and 40 . At these two locations, the computed PSD values show significant overprediction in energy levels at frequencies above 1,000 Hz; higher spatial resolution further increases the magnitude of the differences. A plausible cause for this overestimation may be a difference in the reattachment location for the detached shear layer on the rear strut between the computational and experimental setups. As mentioned earlier, the slightest movement of the separation point on a bluff body could have a profound effect on the shape and amplitude of the pressure fluctuation spectra. Recall that the ultimate test for the simulations is the accuracy of the far field noise prediction.

Sample PSD plots for probes on the gear inner wheel (43 through 45) are presented in Fig. 37. Very good agreement is observed, with the predicted spectra closely tracking the measured data over most of the frequency range. Similarly close agreement was also observed at other probe locations on both wheels (not shown). In general, increased spatial resolution improves the agreement.

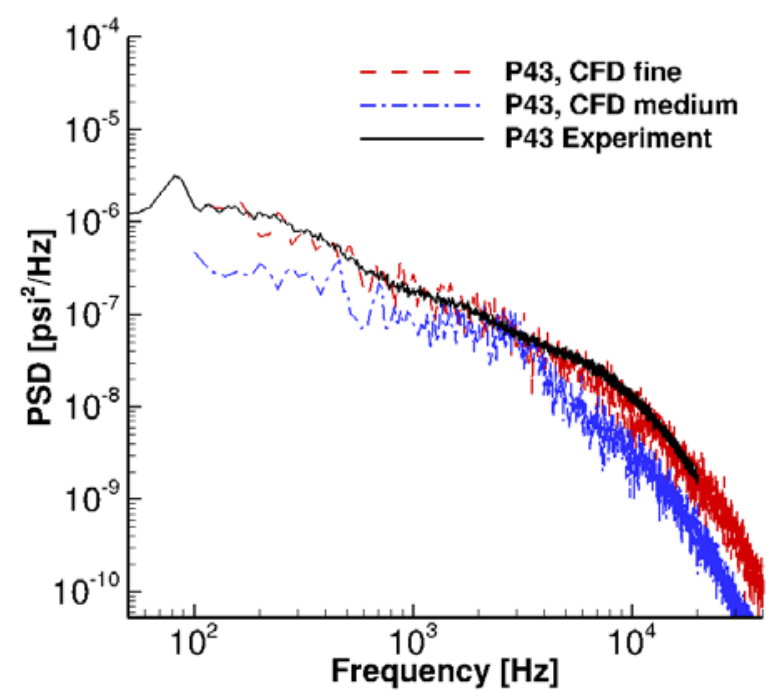

a) Probe 43 location

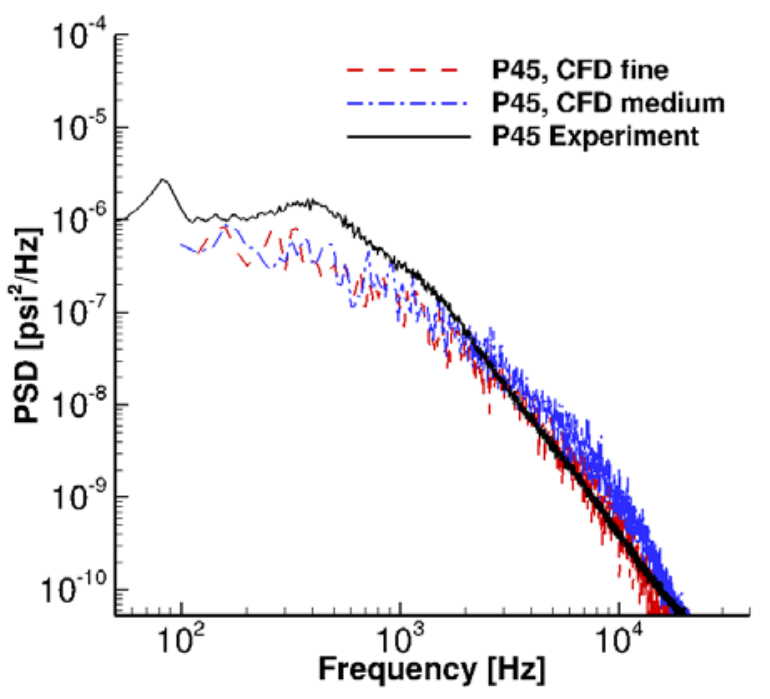

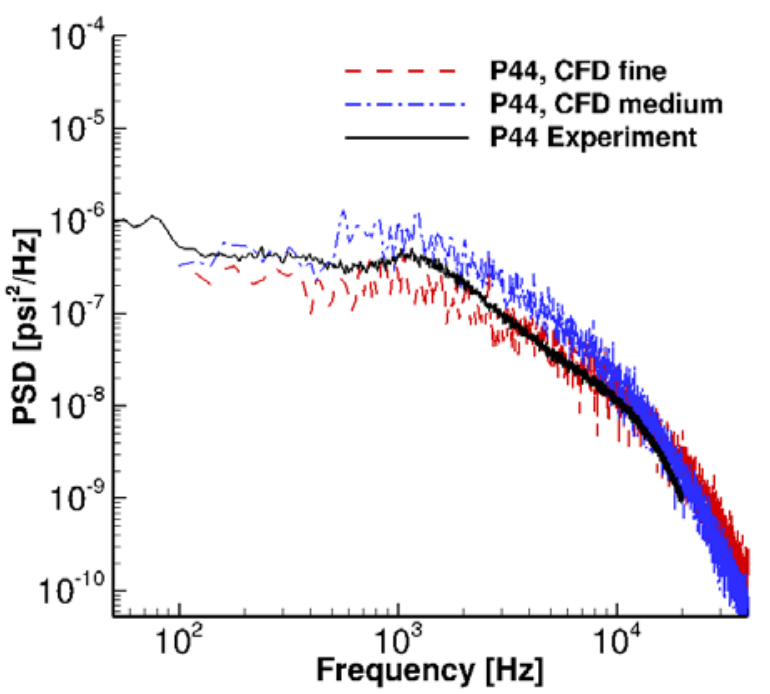

b) Probe 44 location

c) Probe 45 location

Figure 37. Power spectral density of surface pressure fluctuations on main gear inner wheel. 
Surface pressure fluctuation spectra at two locations on the gear door are shown in Fig. 38. The spectra at the selected probes are representative of those observed at other door locations. Notice that the pressure levels on the door are nearly two orders of magnitude lower than those on the other gear components. The simulated PSD curves capture the frequency content and the fluctuation levels up to frequencies approaching $7 \mathrm{kHz}$. At present, the underlying source of the rise in the measured spectra beyond $5-6 \mathrm{kHz}$ is unknown. The increased levels could be caused by hydrodynamic pressure fluctuations associated with the detached flow over the door surface or by the self-noise associated with the pinhole installation of the dynamic sensors. Nevertheless, for this aircraft configuration, the gear door does not appear to be a major noise source and most likely acts as a reflector for the acoustic waves emanating from the flap inboard tip and/or the other components of the gear. As such, notice the presence of several major and minor tones on the door spectra. These tones are generated elsewhere and what is being measured on the door is the resultant radiated pressure field impinging on the surface. The simulations capture most of the tonal features in the measured spectra.

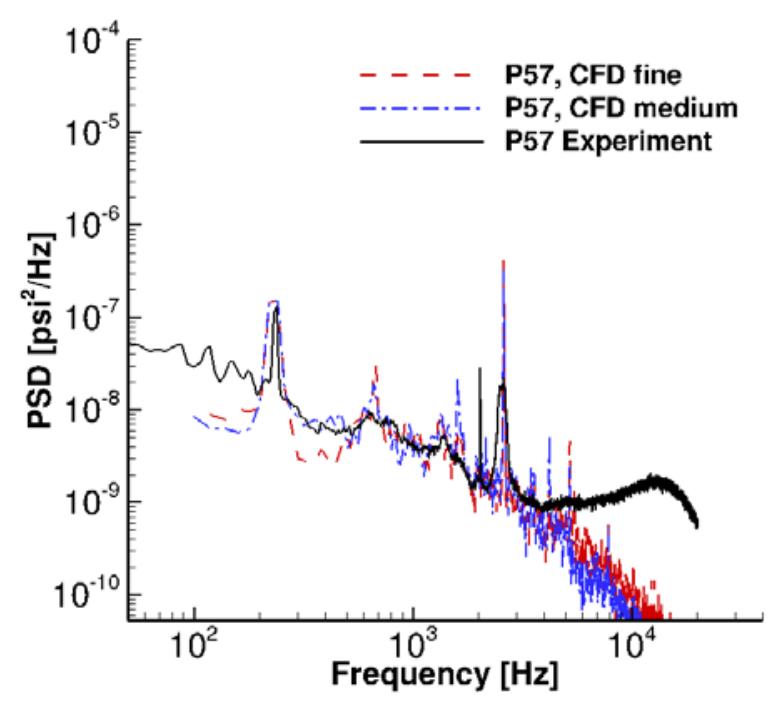

a) Probe 57 location

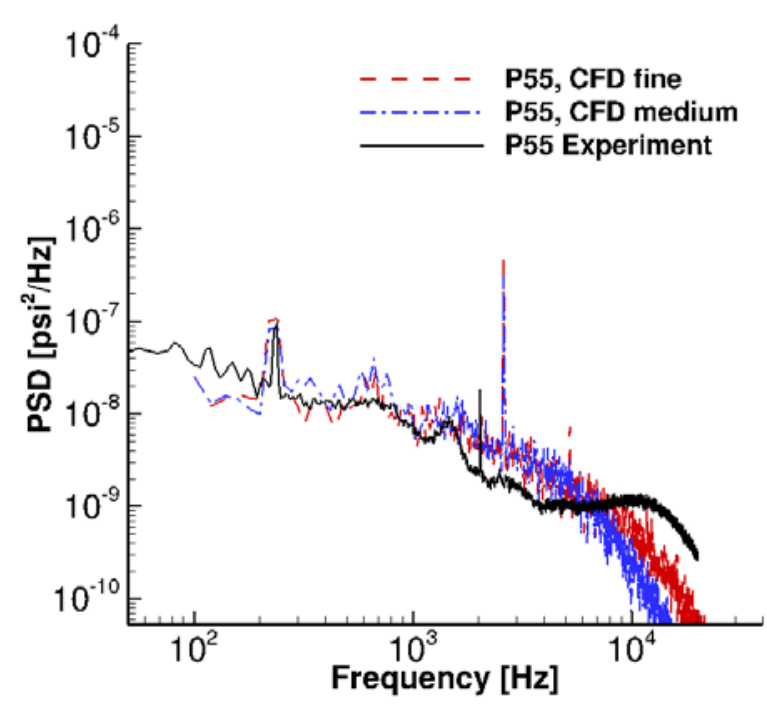

b) probe 55 location

Figure 38. Power spectral density of surface pressure fluctuations on main gear door.

\section{Farfield noise prediction}

The farfield noise computation was conducted using an FWH propagation formulation ${ }^{29}$ on the solid surface of the complete semi-span model with and without the mounting beveled plate (Fig. 39), and on four different permeable surfaces encompassing the complete aircraft, the flap and section of the wing, and both inboard and outboard flap tip regions, as depicted in Fig. 40. The porous surfaces were judiciously oriented in space so as to minimize the hydrodynamic fluctuations crossing through them. The data sampling frequency on the solid surface is $200 \mathrm{kHz}$ and on the porous surfaces is $100 \mathrm{kHz}$. A large volume with resolution supporting a data sampling frequency of $100 \mathrm{kHz}$, as shown in Fig. 39, was also included to allow direct propagation to the farfield microphone located 17.75 feet $(5.334 \mathrm{~m})$ from the model center of rotation. Based on previous investigations, ${ }^{30}$ proper wave propagation within the volume requires about 10 points per wavelength. Thus, the effective grid cut-off frequency for the FWH computations using the porous surfaces is expected to be approximately 10 times lower than the data sampling frequency. Due to the relatively large distance to the farfield microphone, the grid cut-off frequency for the directly simulated probe is expected to be even lower. 


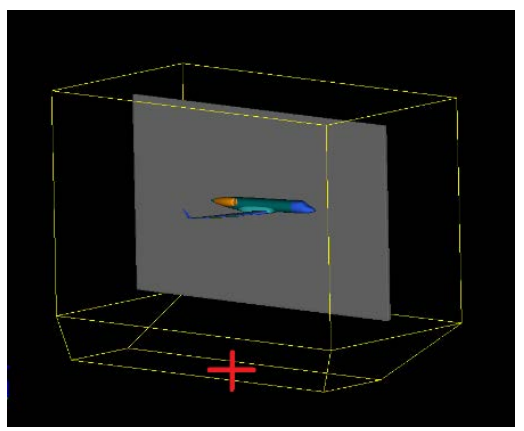

Figure 39. Solid surface, beveled plate, and far field probe location (red cross). The yellow outline region indicates the large high resolution volume.
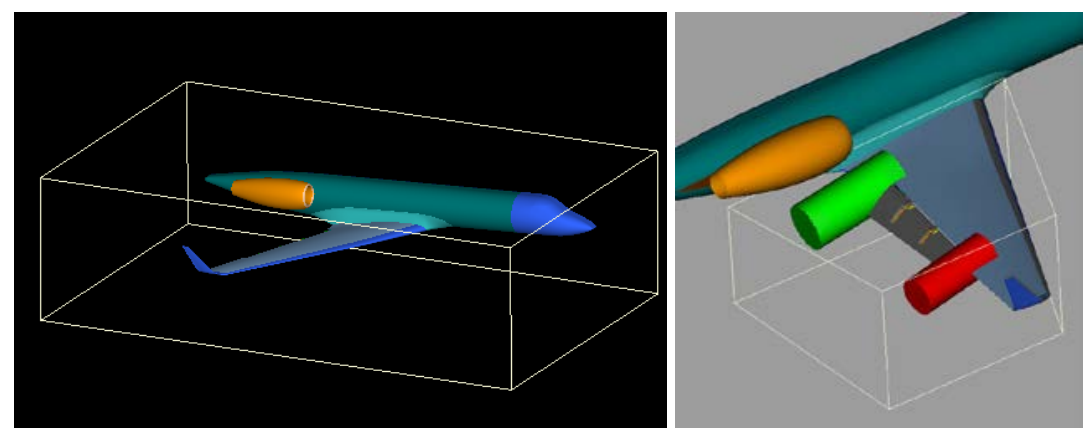

Figure 40. Porous surface around the complete aircraft and portion of the beveled plate (outlined geometry, middle) and around the flap and part of the wing excluding the wingtip (outlined geometry, right) and around the two flap tips (solid green and red cylinders, right)

Acoustic propagation to the farfield probe for the configuration without the main gear is shown in Fig. 41. Good agreement between the FWH calculations using solid surface pressure fluctuations and directly simulated results is observed for frequencies up to the cut off frequency of the fluid volume, approximately $2.5 \mathrm{kHz}$. At higher frequencies, the comparison degrades because of insufficient spatial resolution of the directly calculated fluctuations. The favorable comparison indicates that reflection from the beveled plate and mean-flow convective effects are properly taken into account by the FWH computation. A comparison of three grids with increasing spatial resolution (global refinement factor of 1.5) is presented in Fig. 42 and indicates that the solution gradually approached a converged state. As expected, successively finer grids produce higher intensity levels at higher frequencies. It is interesting to observe that, although the peak magnitude of a tonal feature around $1,500 \mathrm{~Hz}$ was reduced in the finest resolution, the broadband levels in the medium and fine grids are in very good agreement up to about $10 \mathrm{kHz}$. Accordingly, the medium resolution grid with a total of $820 \times 10^{6}$ voxels was considered to be sufficient for the extensive comparative evaluation of different noise reduction concepts that was conducted prior to, and in support of, testing of several airframe noise reduction concepts in the $14 \times 22$ wind tunnel at LaRC. Coverage of the computational effort devoted to that comparative evaluation is beyond the scope of the present paper and will be reported elsewhere in the future.

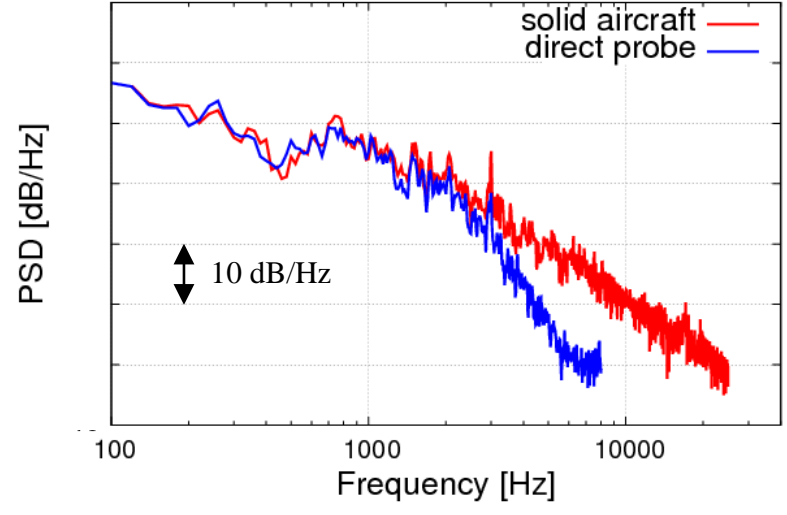

Figure 41. Predicted farfield noise by using solid FWH and direct probe.

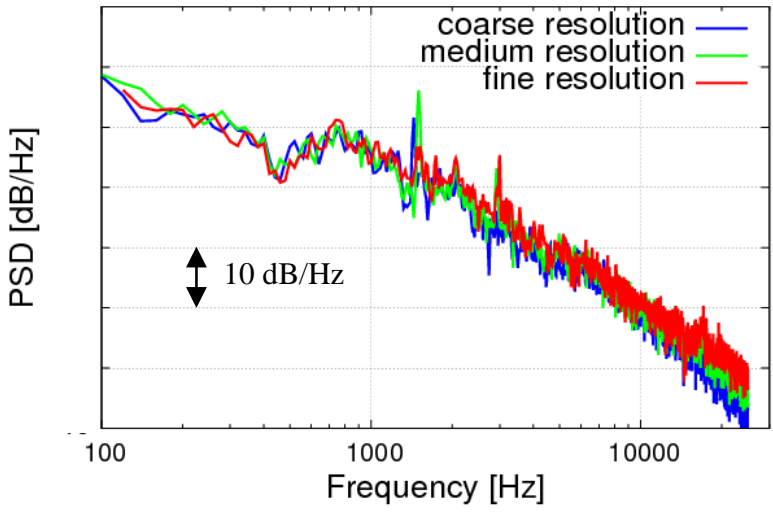

Figure 42. Predicted farfield noise by using solid FWH for different grid resolutions.

Results presented in Fig. 43 provide ample evidence that both solid and porous formulations on the aircraft and the flap region (left plot) are comparable up to a frequency of about $7 \mathrm{kHz}$, with a slight offset in overall PSD level. The region around the flap alone produced almost the same spectrum as the full aircraft porous region and confirms that the flap region is the main noise source in the simulation. The comparison between the two porous FWH cylinders at the inboard and outboard tips (right plot) indicates that the inboard flap tip contributes more to the low frequency range, whereas the outboard flap tip region has a slightly larger effect on the higher frequency content of 
the spectrum. This is expected due to the comparatively smaller geometrical size of the outboard flap tip with respect to the inboard one. Based on the relative contributions of each flap tip region, the mild peak in the aircraft PSD around $1,500 \mathrm{~Hz}$ is most likely associated with the inboard flap tip and the other peak around $2,900 \mathrm{~Hz}$ is more related to the outboard flap tip, and is not a harmonic of the first peak. Surface dB maps (band-integrated wall pressure fluctuations) on the flap tips depicted in Fig. 44 demonstrate that the first peak around 1,500Hz is related to the rather thick trailing edge of the wing and inboard flap section, and very likely associated with the vortex shedding frequency for this edge thickness. The feature at $2,900 \mathrm{~Hz}$ is not as easily identifiable on the surface pressure $\mathrm{dB}$ maps, but it could be related to the shear layer-cavity flow interaction at the outboard flap tip.
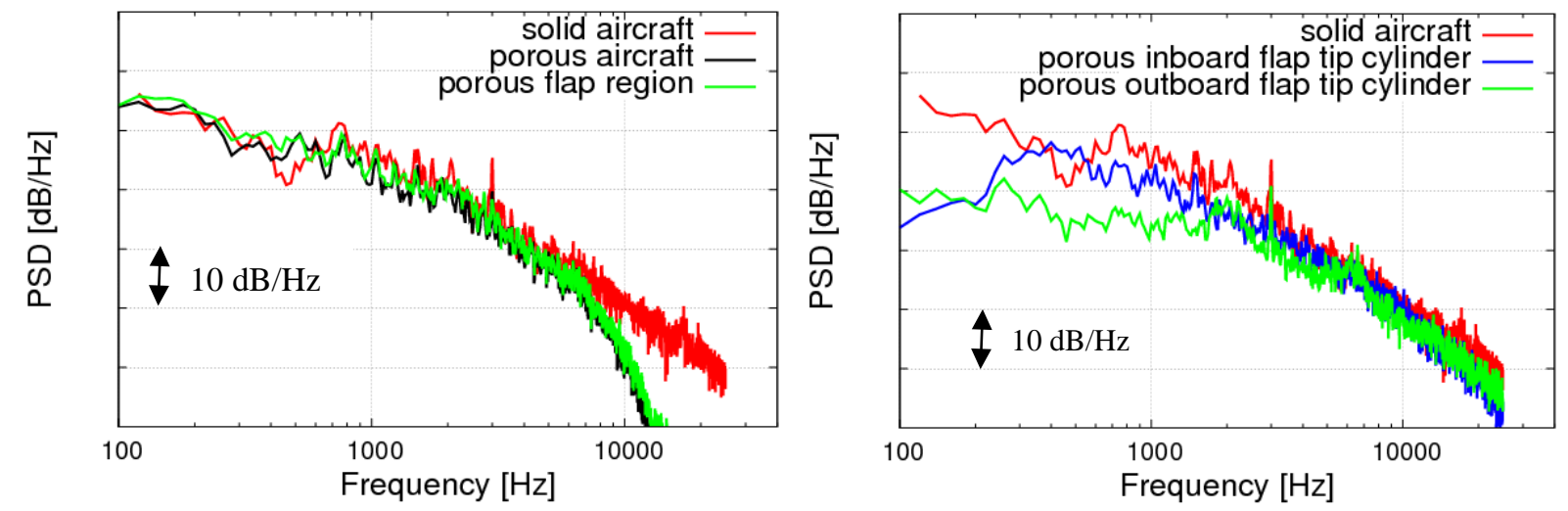

Figure 43. Predicted farfield noise by using FWH for solid and porous formulations.
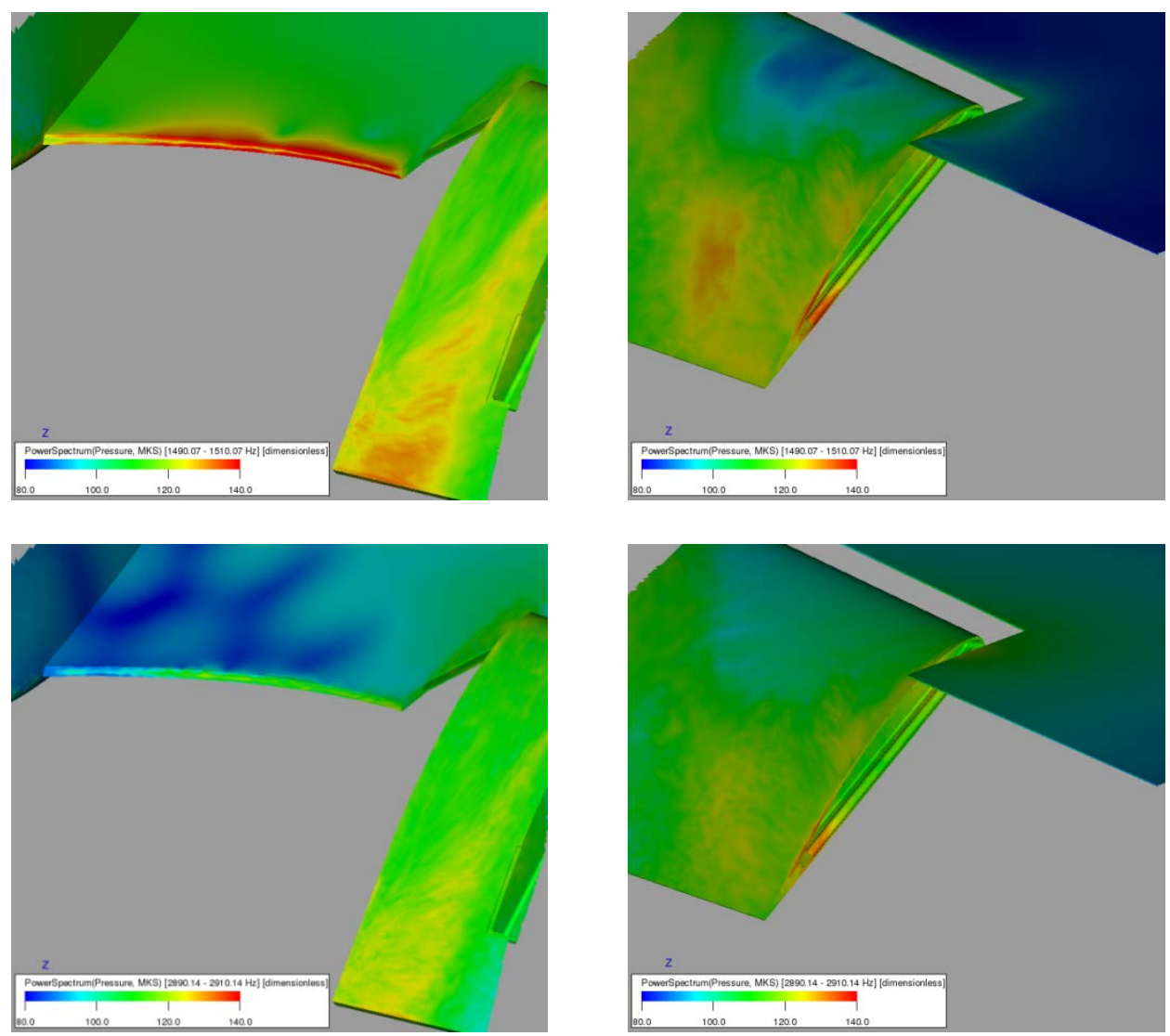

Figure 44. Band-filtered surface pressure power for inboard (left) and outboard (right) flap tips, around $1,500 \mathrm{~Hz}$ (top) and $2,900 \mathrm{~Hz}$ (bottom), for medium resolution grid. 
To conduct a comparative study of predicted vs. measured noise, the FWH-based farfield acoustic computations were performed using pressure fluctuations on the model solid surface (excluding the wind tunnel floor). The simulated unsteady pressure fields on the entire surface of the $18 \%$ scale Gulfstream aircraft model were collected at every 32 time steps for the two baseline configurations, resulting in 51,900 time frames (0.3s record length). The PSD analyses were computed using a $20 \mathrm{~Hz}$ bandwidth, with each block overlapping the previous and following ones (when possible) by $50 \%$.

The farfield noise for the $18 \%$ scale, semi-span Gulfstream aircraft model was obtained recently in the LaRC $14 \times 22$ wind tunnel. As detailed in Khorrami et al., ${ }^{11}$ extensive acoustic measurements were acquired using a phased microphone array. The array was positioned on a set of tracks in the flyover direction $17.5 \mathrm{ft}(5.334 \mathrm{~m})$ away from the model, as shown in Fig. 1. During the test, the traversing array allowed measurements within the $50^{\circ}$ to $110^{\circ}$ polar angles. Noise spectra for $\theta=90^{\circ}$ (overhead) at the corresponding array position are plotted in Fig. 45. Two runs from the 14x22 test displaying the repeatability of the measured noise levels are used in this comparison. The measured spectra were obtained from integration of the microphone array beamform maps corresponding to a region of the tunnel test section covering the entire semi-span model. Although the peak of the noise spectrum resides at frequencies below $200 \mathrm{~Hz}$, the portion of the spectrum relevant to environmental noise concerns corresponds to model scale frequencies of $800 \mathrm{~Hz}$ and above. Because the microphone array was designed for an operational frequency range of approximately $1.5 \mathrm{kHz}-80 \mathrm{kHz}$, data measured below $1,000 \mathrm{~Hz}$ should be used with caution.

The computed far field noise spectrum at the $90^{\circ}$ flyover array position for the baseline case of $39^{\circ}$ flap deflection with the main gear off is compared to the array measurements in Fig. 45. For completeness, the computed spectrum from the companion DES (FUN3D) simulations of Ref. 20 is also shown on the figure. The higher fluctuation amplitude apparent in the computed spectra is related mainly to the shortness of the simulated time records - not enough time segments are available to generate smoother averages that are comparable to those of the test results. Inspection of the far field noise data in Fig. 45 reveals that, for frequencies up to $8 \mathrm{kHz}$, excellent agreement was obtained between both simulations and between each and the measured spectrum - predicted spectrum averages lie within a $\mathrm{dB}$ of the experimental data. At higher frequencies, the PowerFLOW® predicted PSD spectrum closely tracks the broadband component of the farfield noise up to the highest frequency shown (25 $\mathrm{kHz}$ ). Notice the presence of a broad tonal feature in the measurements occurring between $7 \mathrm{kHz}$ and $11 \mathrm{kHz}$ with a peak near $8 \mathrm{kHz}$. This feature is associated with the cavity at the flap outboard tip. Both simulations fail to capture the tonal aspect of the measured spectrum. As stated earlier, precisely matching all the subtle but relevant geometrical details at the outboard tip of the tested model in the simulated geometry is a very difficult task.

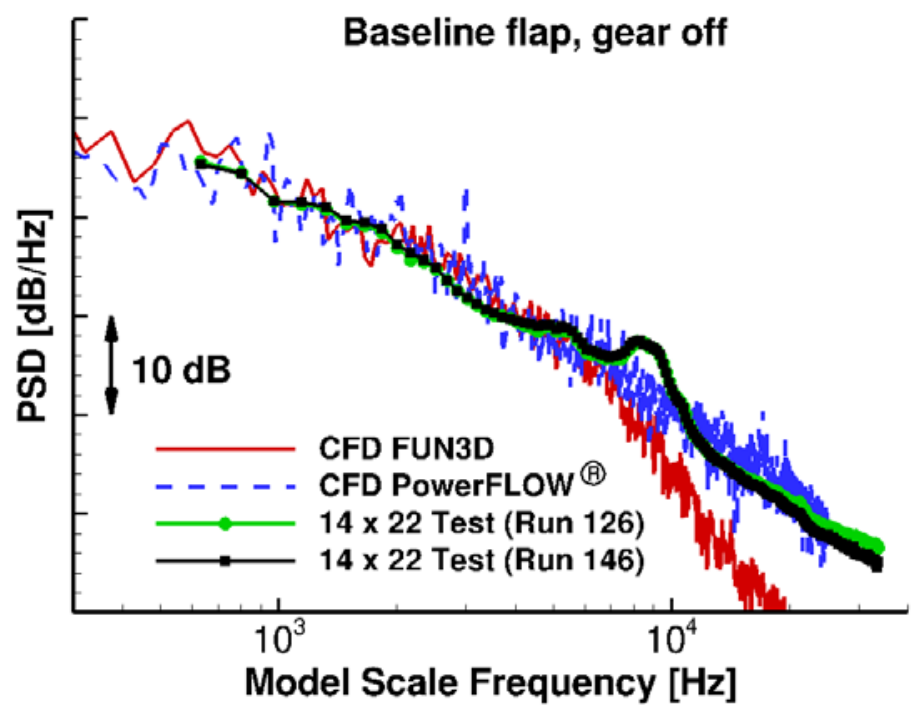

Figure 45. Comparison of farfield noise spectra for landing configuration with $39^{\circ}$ flap deflection and gear off. Computed spectra are based on FWH approach using pressure data on model solid surfaces.

A comparison among predicted and measured noise spectra for the baseline configuration with the main landing gear installed is provided in Fig. 46. As was the case for the flap only configuration, the PSD curves display very good agreement between the two computational approaches and between each simulation and the measured data. 
With the main gear deployed, both computed spectra slightly over-predict the experimental values by about two decibels over most of the frequency range. The very prominent tone centered around $2.5 \mathrm{kHz}$ in the present simulation is produced by the hollowed front post with the cavity opening being exposed at the knee joint. During the acoustic test, after the initial set of runs, this tone was eliminated by taping the cavity face at the joint. Similar action was taken during the grid development stages for the FUN3D-based simulations. For the present simulations, the cavity face was left open to determine how well such tones, in the context of geometries of highest degree of complexity, can be predicted. The results presented in Figs. 45 and 46 provide ample evidence that accurate prediction of the broadband component of airframe noise for a full aircraft is clearly within reach.

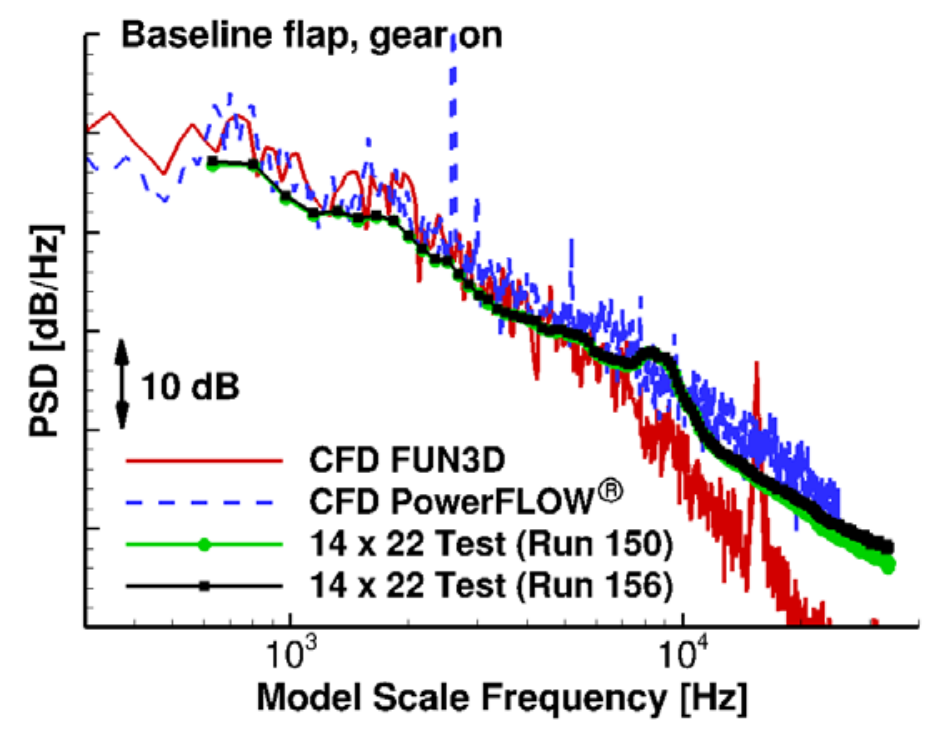

Figure 46. Comparison of farfield noise spectra for landing configuration with $39^{\circ}$ flap deflection and gear on. Computed spectra are based on FWH approach using pressure data on model solid surfaces.

\section{Concluding Remarks}

The present work documents an extensive computational study aimed at advancing simulation-based airframe noise prediction to include a full aircraft geometry in landing configuration. In order to achieve that goal, the computational effort was centered on conducting high-fidelity, time-accurate simulations of an $18 \%$ scale aircraft model that was tested in the NASA LaRC $14 \times 22$ wind tunnel. The as-built model used in the present study is a close replica of a Gulfstream aircraft and features many of the finer details of the full scale geometry.

Exa Corporation's PowerFLOW® lattice Boltzmann solver was used to perform the computations for free-air at a Mach number of 0.2. Experimental results for two baseline landing configurations consisting of $39^{\circ}$ flap deflection with and without the main landing gear deployed were used to validate the computed solutions on medium and fine spatial resolution grids. The baseline configuration with main gear off was used during the initial set of simulations that targeted a) the development of a suitable grid, b) identification of proper mesh clustering/distribution in the critical regions, c) selection of appropriate mesh spacing for resolving pertinent unsteady flow features, and d) assessment of solution dependency on grid characteristics.

Extensive comparisons with available aeroacoustic measurements were performed. The time-averaged surface pressures on the wing and winglet showed excellent agreement with the measured data. Very good agreement was also obtained for the steady pressures at the inboard tip and middle portion of the flap. The dual vortex system and the resulting surface pressure imprint at the inboard tip were well predicted. As in the experiment, the computed pressure coefficients showed a zone of flow separation in the vicinity of the flap outboard tip, with the timeaveraged pressures still containing remnants of low frequency fluctuations within the separation zone. As a result, the agreement between measured and simulated pressures at the outboard tip was not as good as that obtained for the inboard tip. Some of the differences are thought to be caused by the cavity and associated bulb seal, which made duplication of the tested outboard tip geometry inherently difficult.

Predicted surface pressure fluctuation spectra at the flap inboard tip were found to be in remarkably good agreement with the measured data regarding frequency content and spectral shape. Medium and fine spatial 
resolution grids produced nearly the same pressure fluctuation field, indicating that sufficiently accurate predictions can be obtained with the less costly grid.

The configuration with $39^{\circ}$ flap deflection and main landing gear deployed was used to represent a more complete aircraft geometry during landing. Gear-flap interaction effects were shown to be limited to the flap inboard tip region, corroborating measurements of the model in the $14 \times 22$ wind tunnel at NASA LaRC. Changes in the flap steady loading at the inboard tip due to the presence of the gear were predicted accurately. The fluctuating surface pressure spectra at the inboard flap were found to be in excellent agreement with the measured data. As was observed in the experimental data, the computed surface pressure fluctuations on the inboard flap edge side wall and top surface showed elevated levels when the main gear was deployed. Comparison of the predicted fluctuating surface pressure spectra on the landing gear struts, wheels, and door with the measured data revealed good agreement for most of the probe locations shown.

The success of any airframe noise simulation depends on how well the broadband farfield noise spectrum is predicted. For the configuration with the main gear off, the predicted farfield spectrum for the $90^{\circ}$ overhead position was in remarkable agreement with the microphone array measurements. For most of the frequency range shown, the simulation-based acoustic intensities were within $1 \mathrm{~dB}$ of the measured values, falling well within the uncertainty limits of the microphone array. Computed farfield acoustic results for the landing configuration with the main gear on also compared well with measurements. For this configuration, the predicted values were within $2 \mathrm{~dB}$ of the experimental data.

\section{Acknowledgments}

This work was supported by the Environmentally Responsible Aviation (ERA) project under the Integrated System Research Program (ISRP) of NASA. Our thanks go to Scott Brynildsen of Vigyan, Inc., for providing geometry modifications and CAD support. We would also like to express our sincere appreciation for the support provided by Patrick Moran of NASA Ames Research Center for the high quality visualizations and animations of the large data sets. All the simulations were performed on the Pleiades supercomputer at the NASA Advanced Supercomputing (NAS) facility at Ames Research Center. The logistical support provided by NAS staff, in particular Yan-Tyng (Sherry) Chang of Computer Sciences Corporation, is greatly appreciated.

\section{References}

${ }^{1}$ Michel, U., Barsikow, B., Helbig, J., Hellmig, M., and Schüttpelz, M., "Flyover Noise Measurements on Landing Aircraft with a Microphone Array,” AIAA Paper 98-2336, May 1998.

${ }^{2}$ Piet, J.-F., Elias, G., and Lebigot, P., “Localization of Acoustic Sources from a Landing Aircraft with a Microphone Array,” AIAA Paper 99-1811, May 1999.

${ }^{3}$ Pott-Pollenske, M., Dobrzynski, W., Buchholz, H., Gehlhar, B., and Walle, F., "Validation of a Semiempirical Airframe Noise Prediction Method through Dedicated A319 Flyover Noise Measurements,” AIAA Paper 2002-2470, June 2002.

${ }^{4}$ Stoker, R., Guo, Y., Streett, C. L., and Burnside, N., “Airframe Noise Source Locations of a 777 Aircraft in Flight and Comparisons with Past Model Tests,” AIAA Paper 2003-3232, May 2003.

${ }^{5}$ Elkoby, R., Brusniak, L., Stoker, R., Khorrami, M. R., Abeysinghe, A., and Moe, J. W., “Airframe Noise Results from the QTD II Flight Test Program,” AIAA Paper 2007-3457, May 2007.

${ }^{6}$ Khorrami, M. R., Lockard, D. P., Humphreys, Jr., W. M., Choudhari, M. M., and Van de Ven, T., "Preliminary Analysis of Acoustic Measurements from the NASA-Gulfstream Airframe Noise Flight Test,” AIAA Paper 2008-2814, May 2008.

${ }^{7}$ Horne, W. C., James, K. D., Arledge, T. K., Soderman, P. T., Burnside, N., and Jaeger, S. M., “Measurements of 26\%-scale 777 Airframe Noise in the NASA Ames 40- by 80 Foot Wind Tunnel,” AIAA Paper 2005-2810, May 2005.

${ }^{8}$ Jaeger, S. M., Burnside, N. J., Soderman, P. T., Horne, W. C., and James, K. D., "Microphone Array Assessment of an Isolated, 26\%-Scale, High Fidelity Landing Gear,” AIAA Paper 2002-2410, June 2002.

${ }^{9}$ Khorrami, M. R., Hannon, J. A., Neuhart, D. H., Markowski, G. A., and Van de Ven, T., “Aeroacoustic Studies of a HighFidelity Aircraft Model: Part 1—Steady Aerodynamic Measurements,” AIAA Paper 2012-2233, June 2012.

${ }^{10}$ Khorrami, M. R. and Neuhart, D. H., “Aeroacoustic Studies of a High-Fidelity Aircraft Model: Part 2- Unsteady Surface Pressures,” AIAA Paper 2012-2234, June 2012.

${ }^{11}$ Khorrami, M. R., Humphreys, W. M., Lockard, D. P., and Ravetta, P. A., “Aeroacoustic Evaluation of Flap and Landing Gear Noise Reduction Concepts,” Paper to be presented at the $20^{\text {th }}$ AIAA/CEAS Aeroacoustics Conference in Atlanta, GA, June 2014.

${ }^{12}$ Neuhart, D., Hannon, J., and Khorrami, M. R., “Aerodynamic Measurements of a Gulfstream Aircraft Model with and without Noise Reduction Concepts," Paper to be presented at the $20^{\text {th }}$ AIAA/CEAS Aeroacoustics Conference in Atlanta, GA, June 2014. 
${ }^{13}$ Yao, C.-S., Jenkins, L. N, Bartram, S. M., Harris, J., Khorrami, M. R., and Mace, W. D., "Flow-Field Investigation of GearFlap Interaction on a Gulfstream Aircraft Model," Paper to be presented at the $20^{\text {th }}$ AIAA/CEAS Aeroacoustics Conference in Atlanta, GA, June 2014.

${ }^{14}$ Vatsa, V., Lockard, D. P., and Khorrami, M. R., “Application of FUN3D Solver for Aeroacoustic Simulation of a Nose Landing Gear Configuration,” AIAA Paper 2011-2820, June 2011.

${ }^{15}$ Vatsa, V., Lockard, D. P., Khorrami, M. R., and Carlson, J.-R., “Aeroacoustic Simulation of a Nose Landing Gear in an Open-Jet Facility using FUN3D,” AIAA Paper 2012-2280, June 2012.

${ }^{16}$ Li. Y., Satti, R., Lew, P.-T., Shock, R., and Noelting, S., "Computational Aeroacoustic Analysis of Flow Around a Complex Nose Landing Gear Configuration,” AIAA Paper 2008-2916, May 2008.

${ }^{17}$ Keating, A., Dthioux, P., Satti, R., Noelting, S., Louis, J., Van de Ven, T., and Vieito, R., “Computational Aeroacoustics Validation and Analysis of a Nose Landing Gear,” AIAA Paper 2009-3154, May 2009.

${ }^{18}$ Fares, E. and Noelting, S., "Unsteady Flow Simulation of a High-Lift Configuration using a Lattice-Boltzmann Approach," AIAA Paper 2011-0869, January 2011.

${ }^{19}$ Casalino, D., Noelting, S., Fares, E., Vand de Ven, T., Perot, F., and Bres, G., "Towards Numerical Aircraft Noise Certification: Analysis of a Full-Scale Landing Gear in Fly-Over Configuration,” AIAA Paper 2012-2235, June 2012.

${ }^{20}$ Khorrami, M. R. and Mineck, R. E., “Towards Full Aircraft Airframe Noise Prediction: Detached Eddy Simulations,” Paper to be presented at the $20^{\text {th }}$ AIAA/CEAS Aeroacoustics Conference in Atlanta, Georgia, June 2014.

${ }^{21}$ Chen, H., Chen, S., and Matthaeus, W., "Recovery of the Navier-Stokes Equations Using a Lattice-Gas Boltzmann Method,” Physical Review A, Vol. 45, No. 8, 1992, pp.5339-5342.

${ }^{22}$ Lockard, D. "Summary of the Tandem Cylinder Solutions from the Benchmark problems for Airframe Noise Computations-I Workshop,” AIAA Paper 2011-0353, 2011.

${ }^{23}$ Yakhot, V. and Orszag, S. A., “Renormalization Group Analysis of Turbulence. I. Basic Theory,” J. Sci. Comput., Vol 1, No. 2, pp. 3-51, 1986.

${ }^{24}$ Chen, H., Kandasamy, S., Orszag, S.A., Succi, S., and Yakhot, V., "Extended Bolzmann Kinetic Equation for Turbulent Flows,” Science, Vol. 301, No. 5633, 2003, pp- 633-636.

${ }^{25}$ Chen J., "Volumetric formulation of the lattice Boltzmann method for fluid dynamics: Basic concept," Physical Review E, vol. 58, no.3, pp. 3955-3963, 1998.

${ }^{26}$ Chen, H., Teixeira, C., and Molvig, K., "Realization of Fluid Boundary Conditions via Discrete Boltzmann Dynamics," International Journal of Modern Physics C, Vol. 9, No. 8, 1998, pp. 1281-1292.

${ }^{27}$ Ffowcs Williams, J. E. and Hawkings, D. L., "Sound Generated by Turbulence and Surfaces in Arbitrary Motion," Philosophical Transactions of the Royal Society, Vol. A264, No. 1151, pp. 321-342, 1969.

${ }^{28}$ Farassat, F. and Succi, G. P., “The Prediction of Helicopter Discrete Frequency Noise,” Vertica, Vol. 7, No. 4, pp. 309-320, 1983.

${ }^{29}$ Najafi-Yazdi, A., Brès, G. A., and Mongeau, L., “An Acoustic Analogy Formulation for Moving Sources in Uniformly Moving Media,” Proceeding of The Royal Society of London A, Vol. 467 (2125), pp. 144-165, 2011.

${ }^{30}$ Brès, G. A., Pérot, F., and Freed, D. "Properties of the Lattice-Boltzmann Method for Acoustics,” AIAA Paper 2009-3395, May 2009. 